\title{
An Oxidative Dearomatization Approach to Tetrodotoxin via a Masked ortho-Benzoquinone
}

\author{
Jacob G. Robins ${ }^{1}$, Jeffrey S. Johnson ${ }^{1, \star}$ \\ ${ }^{1}$ Department of Chemistry, University of North Carolina at Chapel Hill, Chapel Hill, North Carolina 27599-3290, United States \\ jsj@unc.edu
}

Table of Contents

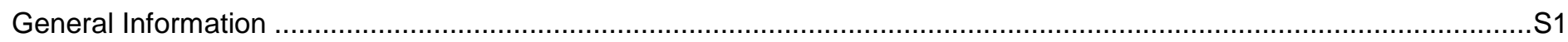

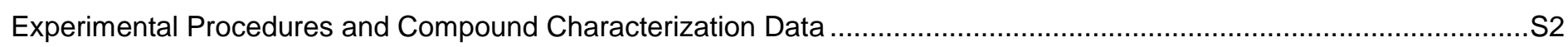

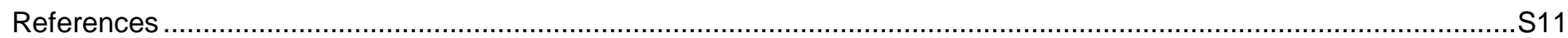

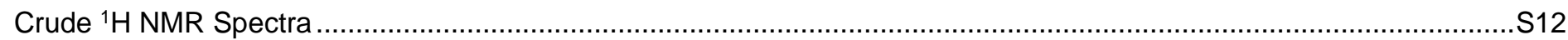

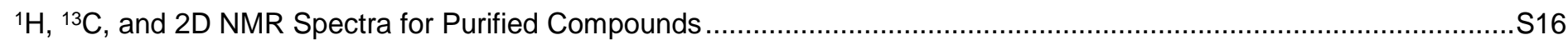

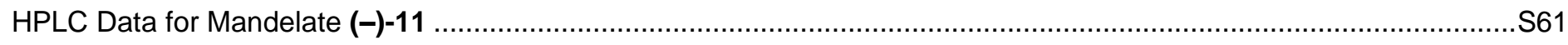

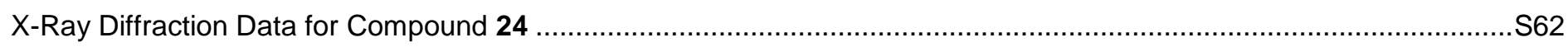

General Information

General Methods: Unless otherwise stated, all reactions were carried out under an atmosphere of dry nitrogen in flamedried glassware with magnetic stirring. Thin layer chromatography (TLC) was performed on Sorbent Technologies $0.20 \mathrm{~mm}$ Silica Gel TLC plates. Visualization was accomplished using UV light and either $\mathrm{KMnO}_{4}$ solution or cerium ammonium molybdate (CAM) solution followed by heating. Flash chromatography was performed under positive air pressure using Siliaflash-P60 silica gel $(40-63 \mu \mathrm{m})$ purchased from Silicycle. Yield refers to isolated yield of pure material unless otherwise noted.

Instrumentation and Data Acquisition: Proton $\left({ }^{1} \mathrm{H}\right)$ and carbon $\left({ }^{13} \mathrm{C}\right)$ magnetic resonance spectra were obtained on Bruker Avance $400 \mathrm{MHz}$, Bruker Avance III $500 \mathrm{MHz}$, Bruker Neo $400 \mathrm{MHz}, \mathrm{NEO} 600 \mathrm{MHz}$, or a Bruker Avance III 600 instruments, using solvent resonances for internal chemical shift calibration $\left({ }^{1} \mathrm{H} N M R: \mathrm{CDCl}_{3}\right.$ at $7.26 \mathrm{ppm}, \mathrm{C}_{6} \mathrm{D}_{6}$ at $7.16 \mathrm{ppm}$, $\mathrm{MeOD}$ at $3.31 \mathrm{ppm} ;{ }^{13} \mathrm{C} \mathrm{NMR}: \mathrm{CDCl}_{3}$ at $77.00 \mathrm{ppm}, \mathrm{C}_{6} \mathrm{D}_{6}$ at $128.06 \mathrm{ppm}, \mathrm{MeOD}$ at $49.00 \mathrm{ppm}$ ). Mass spectra were obtained using a S7 Thermo Scientific Q Exactive HF-X mass spectrometer with electrospray introduction and external calibration. Optical rotations were measured using a $2 \mathrm{~mL}$ cell with a $1 \mathrm{dm}$ path length on a Jasco P-1010 polarimeter. High pressure liquid chromatography was performed using a Perkin Elmer Flexar HPLC system equipped with a Perkin Elmer PDA detector. Daicel Chiralpak IC (received: August 2014) was available for use in separations.

Data Reporting: The following format is used for the presentation of $1 \mathrm{H}$ NMR spectroscopic data: magnet strength, analysis solvent, chemical shift (ppm), multiplicity $(s=$ singlet, $b r s=$ broad singlet, app $s=$ apparent singlet, $d=$ doublet, $b d=b r o a d$ doublet, $\mathrm{t}=$ triplet, app $\mathrm{t}=$ apparent triplet, $\mathrm{q}=$ quartet, app $\mathrm{q}=$ apparent quartet, $\mathrm{dd}=$ doublet of doublets, $t \mathrm{~d}=$ triplet of doublets, app $t d=$ apparent triplet of doublets, $d d d=$ doublet of doublet of doublets, $d d t=$ doublet of doublet of triplets, app $\mathrm{ddt}=$ apparent doublet of doublet of triplets, dddd = doublet of doublet of doublet of doublets, $\mathrm{m}=$ multiplet), $\mathrm{J}$-coupling constants $(\mathrm{Hz})$, and integration.

Materials: Unless otherwise stated, technical grade solvents and reagents were used as received. Anhydrous tetrahydrofuran (THF), diethyl ether $\left(\mathrm{Et}_{2} \mathrm{O}\right)$, methylene chloride $\left(\mathrm{CH}_{2} \mathrm{Cl}_{2}\right)$, toluene $(\mathrm{PhMe})$, and triethylamine $\left(\mathrm{Et}{ }_{3} \mathrm{~N}\right)$ were obtained by passage of the respective solvents through a neutral alumina column under nitrogen pressure. Solvent ratios are reported as volume ratios.

HPLC grade solvents were used for both sample preparation and analysis eluent. 


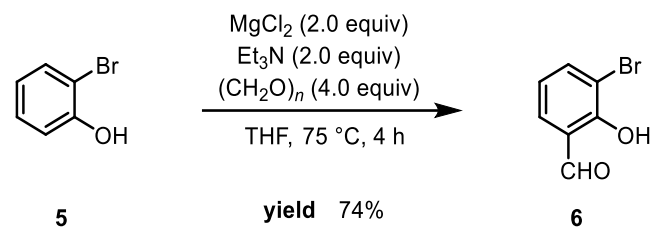

3-Bromo-2-hydroxybenzaldehyde (6): The title compound was prepared via a modified literature procedure. ${ }^{1}$ An ovendried, 3-necked $3000 \mathrm{~mL}$ round-bottomed flask equipped with a large stir bar, a reflux condenser, and a $100 \mathrm{~mL}$ addition funnel was cooled under a stream of nitrogen, suspended in a large oil bath, and charged with magnesium chloride ( 10 mesh beads, >99.9\% pure, $100.0 \mathrm{~g}, 1.050 \mathrm{~mol}, 2.0$ equiv), paraformaldehyde ( $63.09 \mathrm{~g}, 2.101 \mathrm{~mol}, 4.0$ equiv), and dry THF (2.0 L). Dry trimethylamine (146.4 mL, $106.3 \mathrm{~g}, 1.050 \mathrm{mmol}, 2.0$ equiv) was added and the solution was stirred at rt for 10 min. o-Bromophenol ( $60.90 \mathrm{~mL}, 525.2 \mathrm{mmol}, 1.0$ equiv) was charged to the addition funnel and added to the stirred solution over $15 \mathrm{~min}$. The reaction was stirred at $75^{\circ} \mathrm{C}$ (oil bath temperature) for $4 \mathrm{~h}$ then cooled to $\mathrm{rt}$ in an ice bath. The bright yellow solution was diluted with diethyl ether $(400 \mathrm{~mL})$ and transferred to a separatory funnel. The organic layer was washed with $1 \mathrm{M} \mathrm{HCl}(3 \times 300 \mathrm{~mL})$, water $(3 \times 300 \mathrm{~mL})$, and brine $(1 \times 500 \mathrm{~mL})$. The organic layer was dried over sodium sulfate, filtered, and concentrated in vacuo. The resulting crude material was recrystallized from hexanes $\left(\sim 300 \mathrm{~mL}\right.$ at $75^{\circ} \mathrm{C}$ then cooled to $\left.0^{\circ} \mathrm{C}\right)$. A second crop could be obtained from the filtrate to afford the title compound as tan needles $(78.37 \mathrm{~g}, 389.9$ mmol, $74 \%$ ). The spectral data for this compound were in agreement with reported literature values. ${ }^{1}$

Analytical data: ${ }^{1} \mathrm{H}$ NMR $\left(600 \mathrm{MHz}, \mathrm{CDCl}_{3}\right) \delta 11.61(\mathrm{~s}, 1 \mathrm{H}), 9.86(\mathrm{~s}, 1 \mathrm{H}), 7.79(\mathrm{dd}, J=7.9,1.7 \mathrm{~Hz}, 1 \mathrm{H}), 7.55(\mathrm{dd}, J=7.7$, $1.5 \mathrm{~Hz}, 1 \mathrm{H}$ ), 6.95 (dd, $J=7.8,7.7 \mathrm{~Hz}, 1 \mathrm{H}$ ).

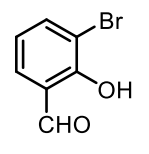

6

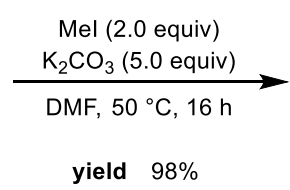

yield $98 \%$

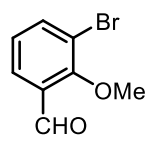

s1

3-Bromo-2-methoxybenzaldehyde (S1): The title compound was prepared via a modified literature procedure..$^{2} \mathrm{~A} 2000$ $\mathrm{mL}$ round-bottomed flask was charged with 3-bromo-2-hydroxybenzaldehyde 6 ( $78.37 \mathrm{~g}, 389.9 \mathrm{mmol}, 1.0$ equiv), DMF (1.95 L), potassium carbonate $(269.4 \mathrm{~g}, 1.949 \mathrm{~mol}, 5.0$ equiv), and methyl iodide ( $48.5 \mathrm{~mL}, 779.7 \mathrm{mmol}, 2.0$ equiv). No attempt was made to exclude air or moisture. The flask was fitted with a reflux condenser and stirred at $50{ }^{\circ} \mathrm{C}$ for $16 \mathrm{~h}$. The suspension was cooled to $\mathrm{rt}$ and filtered through a fritted glass funnel. The carbonate cake was washed with $\mathrm{Et}_{2} \mathrm{O}(2 \times 200$ $\mathrm{mL})$ and the filtrate was diluted with $1 \mathrm{M} \mathrm{HCl}(500 \mathrm{~mL})$. After stirring for $10 \mathrm{~min}$ at $\mathrm{rt}$, the aqueous phase was extracted with $\mathrm{Et}_{2} \mathrm{O}(2 \times 200 \mathrm{~mL})$. The combined organic extracts were washed with saturated aqueous sodium bicarbonate $(1 \times 200 \mathrm{~mL})$, water $(5 \times 50 \mathrm{~mL})$, and brine $(1 \times 200 \mathrm{~mL})$. The organic layer was tried over sodium sulfate, filtered, and concentrated in vacuo. The crude dark red oil was filtered through a plug of silica gel with $15 \%$ EtOAc/Hex and concentrated in vacuo to afford a yellow oil $(78.52 \mathrm{~g}, 365.1 \mathrm{mmol}, 98 \%)$. The spectral data for this compound were in agreement with reported literature values. ${ }^{2}$

Analytical data: ${ }^{1} \mathrm{H}$ NMR $\left(600 \mathrm{MHz}, \mathrm{CDCl}_{3}\right) \delta 10.36(\mathrm{~s}, 1 \mathrm{H}), 7.81(\mathrm{~m}, 2 \mathrm{H}), 7.14(\mathrm{~m}, 1 \mathrm{H}), 3.99(\mathrm{~s}, 3 \mathrm{H})$.

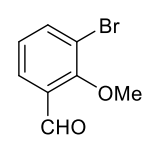

S1

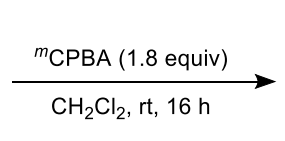

$\mathrm{CH}_{2} \mathrm{Cl}_{2}, \mathrm{rt}, 16 \mathrm{~h}$

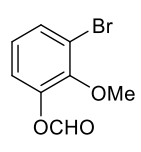

$$
\text { Dow }
$$

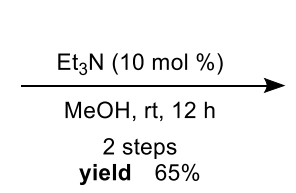

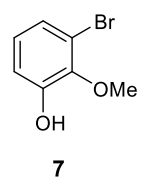

3-Bromo-2-methoxyphenol (7): The title compound was prepared via a modified literature procedure. ${ }^{2}$ Aldehyde $\mathbf{S 1}$ (78.52 $\mathrm{g}, 365.1 \mathrm{mmol}, 1.0$ equiv) was dissolved in $\mathrm{CH}_{2} \mathrm{Cl}_{2}(1.46 \mathrm{~L})$ and treated with $\mathrm{m}$-chloroperbenzoic acid $(151.2 \mathrm{~g}, 657.2 \mathrm{mmol}$, $75 \% \mathrm{w} / \mathrm{w}, 1.8$ equiv). No attempt was made to exclude air or moisture. After stirring at $\mathrm{rt}$ for $16 \mathrm{~h}$, the cloudy white mixture was cooled to $0{ }^{\circ} \mathrm{C}$ and slowly diluted with a mixture of saturated aqueous sodium bicarbonate and saturated aqueous sodium thiosulfate ( 1:1 v/v, $500 \mathrm{~mL}$, CAUTION: GAS EVOLUTION). The aqueous layer was extracted with $\mathrm{Et}_{2} \mathrm{O}(3 \times 200$ $\mathrm{mL}$ ) and the combined ethereal extracts were dried over sodium sulfate, filtered, and concentrated in vacuo. The crude formate was carried directly on to the next step without further purification. 
The dark red oil was dissolved in $\mathrm{MeOH}(1.46 \mathrm{~L})$ and treated with triethylamine (5.09 mL, $36.51 \mathrm{mmol}, 0.10$ equiv). No attempt was made to exclude air or moisture. After stirring at rt for $12 \mathrm{~h}$, the reaction was quenched with $0.1 \mathrm{M} \mathrm{HCl}(385$ $\mathrm{mL})$ and concentrated in vacuo to $\sim 1 / 2$ volume. The aqueous layer was extracted with $\mathrm{Et}_{2} \mathrm{O}(3 \times 200 \mathrm{~mL})$ and the combined ethereal extracts were washed with water $(2 \times 100 \mathrm{~mL})$ and brine $(1 \times 200 \mathrm{~mL})$, dried over sodium sulfate, filtered, and concentrated in vacuo to afford a pale-yellow solid. The crude mixture was dissolved in minimal hexanes at $60^{\circ} \mathrm{C}$, cooled to $0^{\circ} \mathrm{C}$ in an ice-water bath, and filtered through a plug of Celite ${ }^{\circledR}$ to remove residual $m$-chlorobenzoic acid. The filtrate was concentrated in vacuo and filtered through a short plug of silica gel $(\mathrm{EtOAc} / \mathrm{Hex}=5 / 95)$. The crude material was purified by flash column chromatography (500 g silica gel, EtOAc/Hex $=0 / 100$ to $1 / 99$ to $2.5 / 97.5$ to $5 / 95$ ) to afford the title compound as a yellow oil which solidified on standing at $-20^{\circ} \mathrm{C}(48.12 \mathrm{~g}, 237.0 \mathrm{mmol}, 65 \%$ yield over two steps $)$. The spectral data for this compound were in agreement with reported literature values. ${ }^{2}$

Analytical data: ${ }^{1} \mathrm{H}$ NMR $\left(400 \mathrm{MHz}, \mathrm{CDCl}_{3}\right) \delta 7.06(\mathrm{dd}, J=7.6,2.0 \mathrm{~Hz}, 1 \mathrm{H}), 5.72(\mathrm{~s}, 1 \mathrm{H}), 3.91(\mathrm{~s}, 3 \mathrm{H}) ; \mathrm{TLC}(\mathrm{EtOAc} / \mathrm{Hex}=$ 10/90): $R_{f}=0.15$.
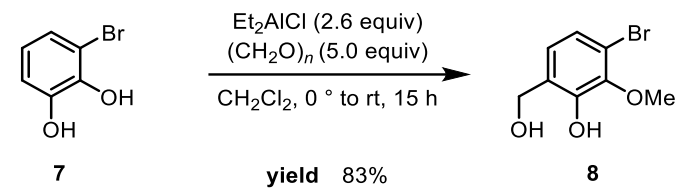

3-Bromo-6-(hydroxymethyl)-2-methoxyphenol (8): These conditions were adapted from previous reports. ${ }^{3-5}$ An ovendried 2-necked $2000 \mathrm{~mL}$ round-bottomed flask equipped with a stir bar and an oven-dried pressure-equalizing addition funnel was cooled under a stream of nitrogen and charged with bromoguaiacol 7 (25.16 g, $123.9 \mathrm{mmol}, 1.0$ equiv), paraformaldehyde $\left(18.61 \mathrm{~g}, 619.9 \mathrm{mmol}, 5\right.$ equiv), and $\mathrm{CH}_{2} \mathrm{Cl}_{2}(335 \mathrm{~mL})$. The suspension was cooled to $0{ }^{\circ} \mathrm{C}$. A freshly

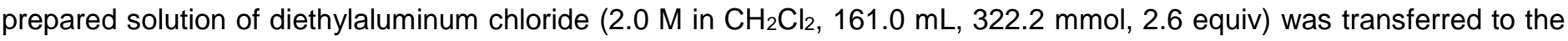
addition funnel via cannula addition then added dropwise to the reaction mixture over $30 \mathrm{~min}$. The reaction was allowed to slowly warm to rt overnight. The resulting mixture was cooled to $0^{\circ} \mathrm{C}$ and slowly quenched with $1 \mathrm{M} \mathrm{HCl}(300 \mathrm{~mL}, \mathrm{CAUTION}$ : GAS EVOLUTION). The aqueous layer was extracted with EtOAc $(3 \times 300 \mathrm{~mL})$ and the combined organic extracts were washed with water $(1 \times 200 \mathrm{~mL})$ and brine $(1 \times 200 \mathrm{~mL})$, dried over sodium sulfate, filtered, and concentrated in vacuo to afford a pale-yellow solid. The resulting crude material was recrystallized from $\mathrm{CH}_{2} \mathrm{Cl}_{2} /$ hexanes and a second crop could be obtained from the filtrate to afford the title compound as a white powder $(23.84 \mathrm{~g}, 102.3 \mathrm{mmol}, 83 \%)$.

Analytical data: $\mathrm{mp} 86-88^{\circ} \mathrm{C} ;{ }^{1} \mathrm{H}$ NMR $\left(600 \mathrm{MHz}, \mathrm{CDCl}_{3}\right) \delta 7.03(\mathrm{~d}, J=8.2 \mathrm{~Hz}, 1 \mathrm{H}), 6.86(\mathrm{~d}, J=8.3 \mathrm{~Hz}, 1 \mathrm{H}), 6.61(\mathrm{br} \mathrm{s}$, $1 \mathrm{H}), 4.71(\mathrm{~d}, J=4.7 \mathrm{~Hz}, 2 \mathrm{H}), 3.90(\mathrm{~s}, 3 \mathrm{H}), 2.46(\mathrm{br} \mathrm{s}, 1 \mathrm{H}) ;{ }^{13} \mathrm{C}\left\{{ }^{1} \mathrm{H}\right\} \mathrm{NMR}\left(151 \mathrm{MHz}, \mathrm{CDCl}_{3}\right) \delta$ 148.5, 144.4, 126.7, 124.7, 123.9, 115.5, 61.8, 61.0; HRMS (ESI+) m/z: [M + Na] ${ }^{+}$Calcd. for $\mathrm{C}_{8} \mathrm{H}_{9}{ }^{35} \mathrm{BrO}_{3} \mathrm{Na}$ 254.9633; Found 254.9626; Calcd. for $\left[\mathrm{C}_{8} \mathrm{H}_{9}{ }^{37} \mathrm{BrO}_{3}+\mathrm{Na}\right]^{+}:$: 256.9607, Found: 256.9605; IR (thin film, $\mathrm{cm}^{-1}$ ): 3422, 3301, 3194, 2948, 1457, 1421, 1008, 877, 796; $\operatorname{TLC}(\mathrm{EtOAc} / \mathrm{Hex}=30 / 70): \mathrm{R}_{f}=0.15$.
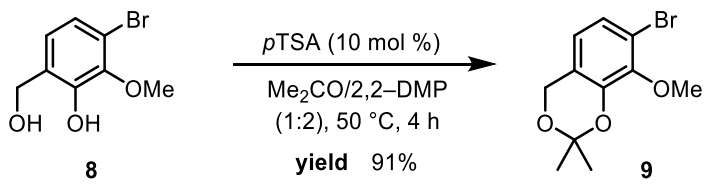

7-Bromo-8-methoxy-2,2-dimethyl-4H-benzo[d][1,3]dioxine (9): A $500 \mathrm{~mL}$ round-bottomed flask equipped with a stir bar and a reflux condenser was charged with salicyl alcohol 8 (17.65 g, $75.73 \mathrm{mmol}, 1.0$ equiv), acetone (125 mL), dimethoxypropane $(250 \mathrm{~mL})$, and $p$-toluenesulfonic acid monohydrate $(1.440 \mathrm{~g}, 7.573 \mathrm{mmol}, 0.10 \mathrm{equiv})$. No attempt was made to exclude air or moisture. The mixture was stirred at $50{ }^{\circ} \mathrm{C}$ (oil bath temperature) for $4 \mathrm{~h}$ then cooled to rt and quenched with saturated aqueous sodium bicarbonate. The aqueous layer was extracted with EtOAc $(3 \times 200 \mathrm{~mL})$ and the combined organic extracts were washed with brine, dried over sodium sulfate, filtered, and concentrated in vacuo to afford a clear, colorless oil. The crude material was purified by flash column chromatography (silica gel, EtOAc/ Hex $=5 / 95$ ) to provide acetonide 9 as a clear, colorless oil $(18.84 \mathrm{~g}, 68.98 \mathrm{mmol}, 91 \%)$.

Analytical data: ${ }^{1} \mathrm{H}$ NMR $\left(600 \mathrm{MHz}, \mathrm{CDCl}_{3}\right) \delta 7.05(\mathrm{~d}, J=8.3 \mathrm{~Hz}, 1 \mathrm{H}), 6.60(\mathrm{dt}, J=8.3,1.0 \mathrm{~Hz}, 1 \mathrm{H}), 4.79(\mathrm{~d}, J=1.0 \mathrm{~Hz}$, 2H), $3.85(\mathrm{~s}, 3 \mathrm{H}), 1.57(\mathrm{~s}, 6 \mathrm{H}) ;{ }^{13} \mathrm{C}\left\{{ }^{1} \mathrm{H}\right\}$ NMR $\left(151 \mathrm{MHz}, \mathrm{CDCl}_{3}\right) \delta 145.6,145.4,123.7,120.4,120.1,115.4,100.1,60.48$, 60.46, 24.7; HRMS (ESI+) m/z: [M + Na] ${ }^{+}$Calcd. for $\mathrm{C}_{11} \mathrm{H}_{13}{ }^{79} \mathrm{BrO}_{3} \mathrm{Na}$ : 294.9941; Found 294.9939; m/z: [M + Na] ${ }^{+}$Calcd. for 
$\mathrm{C}_{11} \mathrm{H}_{13}{ }^{81} \mathrm{BrO}_{3} \mathrm{Na}$ 296.9920; Found 296.9918; IR (thin film, $\mathrm{cm}^{-1}$ ): 2994, 2940, 2858, 2828, 1456, 1423, 1358, 1062, 1018, 906; TLC $(\mathrm{EtOAc} / \mathrm{Hex}=10 / 90): \mathrm{R}_{f}=0.25$.
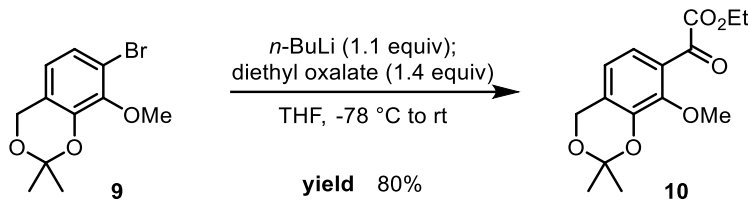

Ethyl 2-(8-methoxy-2,2-dimethyl-4H-benzo[d][1,3]dioxin-7-yl)-2-oxoacetate (10): A flame-dried 2-necked $1000 \mathrm{~mL}$ round-bottomed flask equipped with a stir bar was cooled under a stream of nitrogen and charged with bromide 9 (18.97 g, $69.450 \mathrm{mmol}, 1.0$ equiv) and THF ( $300 \mathrm{~mL})$. The mixture was cooled to $-78{ }^{\circ} \mathrm{C}$ and a solution of $n$-butyllithium $(2.50 \mathrm{M}$ in hexanes, $30.6 \mathrm{~mL}, 76.4 \mathrm{mmol}, 1.1$ equiv) was added dropwise over $10 \mathrm{~min}$. The orange solution was stirred at the same temperature for $10 \mathrm{~min}$ then neat diethyl oxalate $(13.21 \mathrm{~mL}, 97.24 \mathrm{mmol}, 1.4$ equiv) was added dropwise over $10 \mathrm{~min}$. After stirring at the same temperature for $20 \mathrm{~min}$, the reaction was quenched with saturated aqueous ammonium chloride and warmed to rt. The aqueous layer was extracted with EtOAc $(3 \times 200 \mathrm{~mL})$ and the combined organic extracts were washed with brine, dried over sodium sulfate, filtered, and concentrated in vacuo to afford a pale-yellow oil. The crude material was purified by flash column chromatography (silica gel, EtOAc/ Hex $=0 / 100$ to $5 / 95$ to 10/90) to provide $\alpha$-ketoester 10 as a pale-yellow oil (16.23 g, $55.15 \mathrm{mmol}, 80 \%)$.

Analytical data: ${ }^{1} \mathrm{H}$ NMR $\left(600 \mathrm{MHz}, \mathrm{CDCl}_{3}\right) \delta 7.43(\mathrm{dd}, J=8.1,0.7 \mathrm{~Hz}, 1 \mathrm{H}), 6.82(\mathrm{dd}, J=8.1,1.0 \mathrm{~Hz}, 1 \mathrm{H}), 4.87(\mathrm{~s}, 2 \mathrm{H}), 4.39$ (q, $J=7.2 \mathrm{~Hz}, 2 \mathrm{H}), 3.87(\mathrm{~s}, 3 \mathrm{H}), 1.58(\mathrm{~s}, 6 \mathrm{H}), 1.39(\mathrm{t}, J=7.2 \mathrm{~Hz}, 3 \mathrm{H}) ;{ }^{13} \mathrm{C}\left\{{ }^{1} \mathrm{H}\right\} \mathbf{N M R}\left(151 \mathrm{MHz}, \mathrm{CDCl}_{3}\right) \delta 186.5,165.3,150.1$, 144.2, 127.9, 125.6, 120.5, 119.8, 100.4, 61.9, 61.4, 60.8, 24.7, 14.1; HRMS (ESI+) m/z: [M + Na]+ Calcd. for $\mathrm{C}_{15} \mathrm{H}_{18} \mathrm{O}_{6} \mathrm{Na}^{+}$ 317.1001; Found 317.0987; IR (thin film, $\mathrm{cm}^{-1}$ ): 2993, 2945, 2860, 1737, 1674, 1466, 1257, 1215, 1082, 957; TLC $(\mathrm{EtOAc} / \mathrm{Hex}=15 / 85): \mathrm{R}_{f}=0.27$.

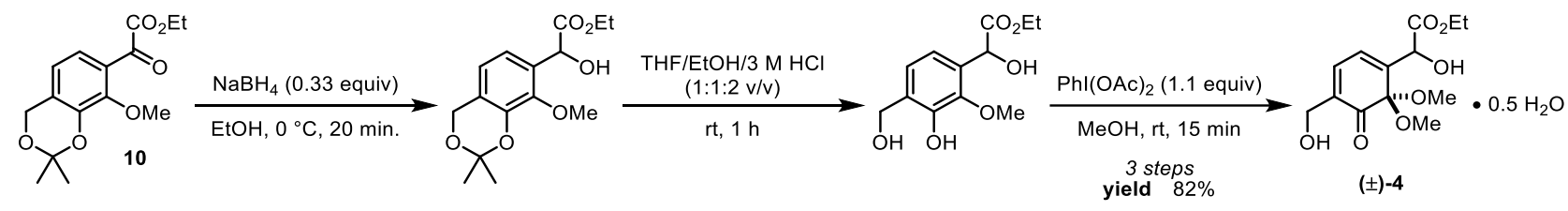

( \pm )-Ethyl 2-hydroxy-2-(4-(hydroxymethyl)-6,6-dimethoxy-5-oxocyclohexa-1,3-dien-1-yl)acetate (4): A solution of $\alpha$ ketoester 10 (11.77 g, $39.99 \mathrm{mmol}, 1.0$ equiv) in $\mathrm{EtOH}(80 \mathrm{~mL})$ was cooled to $0{ }^{\circ} \mathrm{C}$ and charged with sodium borohydride ( $453.9 \mathrm{mg}, 12.00 \mathrm{mmol}, 0.3$ equiv). No attempt was made to exclude air or moisture. After stirring for $20 \mathrm{~min}$, the reaction was quenched with saturated aqueous ammonium chloride and concentrated in vacuo to approx. $1 / 2$ volume. The aqueous phase was extracted with EtOAc $(3 \times 200 \mathrm{~mL})$ and the combined organic extracts were washed with brine, dried over sodium sulfate, filtered, and concentrated in vacuo to afford an opaque colorless oil. The crude material was judged by ${ }^{1} \mathrm{H}$ NMR spectroscopy to be sufficiently pure and was carried forward without further purification. Analytical data: ${ }^{1} \mathbf{H ~ N M R}(400 \mathrm{MHz}$, $\left.\mathrm{CDCl}_{3}\right) \delta 6.82(\mathrm{~d}, J=7.9 \mathrm{~Hz}, 1 \mathrm{H}), 6.72(\mathrm{~d}, J=8.0 \mathrm{~Hz}, 1 \mathrm{H}), 5.25(\mathrm{~d}, J=6.1 \mathrm{~Hz}, 1 \mathrm{H}), 4.84(\mathrm{~s}, 2 \mathrm{H}), 4.29(\mathrm{dq}, J=10.6,7.1 \mathrm{~Hz}$, $1 \mathrm{H}), 4.17(\mathrm{dq}, J=10.7,7.1 \mathrm{~Hz}, 1 \mathrm{H}), 3.87(\mathrm{~s}, 3 \mathrm{H}), 3.61(\mathrm{~d}, J=6.1 \mathrm{~Hz}, 1 \mathrm{H}), 1.23(\mathrm{t}, J=7.1 \mathrm{~Hz}, 3 \mathrm{H})$; TLC $(\mathrm{EtOAc} / \mathrm{Hex}=$ 20/80): $\mathrm{R}_{f}=0.15$.

A $500 \mathrm{~mL}$ round-bottomed flask was charged sequentially with crude $\alpha$-hydroxyester, THF (49 mL), EtOH (49 mL), and $3 \mathrm{M} \mathrm{HCl}(97 \mathrm{~mL})$. No attempt was made to exclude air or moisture. After stirring at rt for $1 \mathrm{~h}$, the solution was diluted with brine $(250 \mathrm{~mL})$ and the aqueous phase was extracted with EtOAc $(6 \times 100 \mathrm{~mL})$. The combined organic extracts were washed with brine, dried over sodium sulfate, filtered, and concentrated in vacuo to afford a white solid. The crude material was judged by ${ }^{1} \mathrm{H}$ NMR spectroscopy to be sufficiently pure and was carried forward without further purification. Analytical data: ${ }^{1} \mathrm{H}$ NMR $\left(400 \mathrm{MHz}, \mathrm{CDCl}_{3}\right) \delta 6.96(\mathrm{br} \mathrm{s}, 1 \mathrm{H}), 6.88(\mathrm{~d}, J=7.9 \mathrm{~Hz}, 1 \mathrm{H}), 6.77(\mathrm{~d}, J=7.9 \mathrm{~Hz}, 1 \mathrm{H}), 5.28(\mathrm{~s}, 1 \mathrm{H}), 4.78(\mathrm{~s}$, 2H), $4.28(\mathrm{dq}, J=10.8,7.1 \mathrm{~Hz}, 1 \mathrm{H}), 4.18(\mathrm{dq}, J=10.8,7.1 \mathrm{~Hz}, 1 \mathrm{H}), 3.89(\mathrm{~s}, 3 \mathrm{H}), 2.04(\mathrm{~s}, 1 \mathrm{H}), 1.22(\mathrm{t}, J=7.1 \mathrm{~Hz}, 3 \mathrm{H})$; TLC $(\mathrm{EtOAc} / \mathrm{Hex}=60 / 40): \mathrm{R}_{f}=0.15$.

A $500 \mathrm{~mL}$ round-bottomed flask was charged sequentially with crude diol, $\mathrm{MeOH}(180 \mathrm{~mL})$ and (diacetoxyiodo)benzene (12.93 g, $40.15 \mathrm{mmol}, 1.1$ equiv). No attempt was made to exclude air or moisture. After stirring at rt for $15 \mathrm{~min}$, the bright yellow solution was concentrated in vacuo. The crude material was immediately purified by flash 
column chromatography (silica gel, EtOAc/Hex $=50 / 50$ to $60 / 40$ to $80 / 20$ ) to afford the title compound as a dark orange oil (10.93 g, $32.69 \mathrm{mmol}, 82 \%$ over three steps).

Analytical data: ${ }^{1} \mathrm{H}$ NMR $\left(600 \mathrm{MHz}, \mathrm{CDCl}_{3}\right) \delta 6.93(\mathrm{dt}, J=6.3,1.5 \mathrm{~Hz}, 1 \mathrm{H}), 6.50(\mathrm{~d}, J=6.3 \mathrm{~Hz}, 1 \mathrm{H}), 4.83(\mathrm{~d}, J=7.6 \mathrm{~Hz}$, $1 \mathrm{H}), 4.39(\mathrm{~s}, 2 \mathrm{H}), 4.28(\mathrm{dq}, J=10.8,7.1 \mathrm{~Hz}, 1 \mathrm{H}), 4.20(\mathrm{dq}, J=10.8,7.1 \mathrm{~Hz}, 1 \mathrm{H}), 3.48(\mathrm{~d}, J=7.8 \mathrm{~Hz}, 1 \mathrm{H}), 3.28(\mathrm{~s}, 3 \mathrm{H}), 3$. 25 (s, 3H), 2.34 (br s, 1H), 1.77 (br s, $1 \mathrm{H}), 1.28(\mathrm{t}, J=7.1 \mathrm{~Hz}, 3 \mathrm{H}) ;{ }^{13} \mathrm{C}\left\{{ }^{1} \mathrm{H}\right\}$ NMR $\left(151 \mathrm{MHz}, \mathrm{CDCl}_{3}\right) \delta$ 196.7, 172.2, 145.4, 126.4, 135.4, 126.1, 93.8, 10.1, 62.0, 59.8, 51.3, 50.9, 14.1; HRMS (ESI) m/z: [M - H] - Calcd. for $\mathrm{C}_{13} \mathrm{H}_{17} \mathrm{O}_{7}$ 285.0980; Found 2855.0981; IR (thin film, cm-1): 3447, 3055, 2986, 2944, 2837, 1735, 1671, 1265, 1075, 739; TLC (EtOAc/Hex = 70/30): Rf $=0.30$.
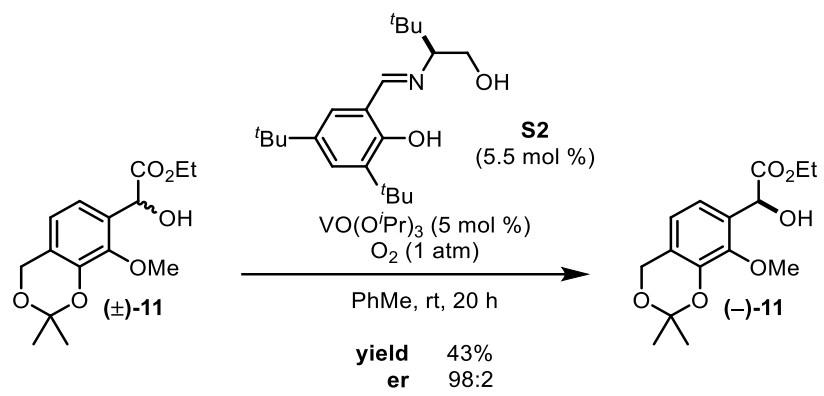

Synthesis of Enantioenriched Mandelate (-)-11: These conditions were adapted from previous reports. ${ }^{6,7} \mathrm{~A}$ flame-dried 1-dram vial was cooled under a stream of $\mathrm{N}_{2}$, brought into a nitrogen-filled glovebox, and charged with salen ligand S2 (3.7 $\mathrm{mg}, 0.011 \mathrm{mmol}, 0.055$ equiv) and dry $\mathrm{PhMe}(0.5 \mathrm{~mL})$. The yellow solution was treated with $\mathrm{VO}\left(\mathrm{O}^{\prime} \mathrm{Pr}\right)_{3}(2.36 \mu \mathrm{L}, 0.010 \mathrm{mmol}$, 0.05 equiv). The resulting brown solution was brought out of the nitrogen-filled glovebox and stirred under an atmosphere of $\mathrm{O}_{2}$ for $30 \mathrm{~min}$. Then, a solution of racemic alcohol ( $\left.\mathbf{\pm}\right)-\mathbf{1 1}(59.3 \mathrm{mg}, 0.200 \mathrm{mmol}, 1.0$ equiv) in dry PhMe $(0.5 \mathrm{~mL})$ was added by syringe. After stirring at it for $20 \mathrm{~h}$, the brown solution was filtered through a plug of silica gel with diethyl ether (6 $\mathrm{mL}$ ) and the filtrate was concentrated in vauco. The crude material was purified by flash column chromatography (silica gel, $\mathrm{EtOAc} / \mathrm{Hex}=5 / 95$ to $10 / 90$ to $20 / 80$ ) to provide mandelate $(-)-11$ as a colorless oil $(25.5 \mathrm{mg}, 0.0861 \mathrm{mmol}, 43 \%)$.

Analytical data: ${ }^{1} \mathbf{H}$ NMR $\left(600 \mathrm{MHz}, \mathrm{CDCl}_{3}\right) \delta 6.82(\mathrm{~d}, J=7.9 \mathrm{~Hz}, 1 \mathrm{H}), 6.72(\mathrm{dt}, J=7.9,0.9 \mathrm{~Hz}, 1 \mathrm{H}), 5.25(\mathrm{~d}, J=6.2 \mathrm{~Hz}$, $1 \mathrm{H}), 4.84(\mathrm{~s}, 2 \mathrm{H}), 4.29(\mathrm{dq}, J=10.8,7.1 \mathrm{~Hz}, 1 \mathrm{H}), 4.17(\mathrm{dq}, J=10.8,7.1 \mathrm{~Hz}, 1 \mathrm{H}), 3.87(\mathrm{~s}, 3 \mathrm{H}), 3.60(\mathrm{~d}, J=6.3 \mathrm{~Hz}, 1 \mathrm{H}), 1.58$ $(\mathrm{s}, 8 \mathrm{H}), 1.23(\mathrm{t}, J=7.1 \mathrm{~Hz}, 3 \mathrm{H}) ;{ }^{13} \mathrm{C}\left\{{ }^{1} \mathrm{H}\right\} \mathrm{NMR}\left(151 \mathrm{MHz}, \mathrm{CDCl}_{3}\right) \delta 173.8,146.3,144.3,130.8,121.4,119.7,119.5,99.9$, 69.6, 62.0, 60.9, 60.7, 24.8, 24.7, 14.1; HRMS (ESI) m/z: $[\mathrm{M}+\mathrm{Na}]^{+}$Calcd. for $\mathrm{C}_{15} \mathrm{H}_{20} \mathrm{O}_{6} \mathrm{Na}$ 319.1158, Found: 319.1147; HPLC (Daicel CHIRALPAK IC, iso-propanol $/ n$-hexane $=10 / 90$, flow rate: $1.0 \mathrm{~mL} / \mathrm{min}, \mathrm{I}=205 \mathrm{~nm}$ ) $\mathrm{t}_{R}=17.3 \mathrm{~min}$ (major), 21.2 min (minor); $[\alpha]_{D}:-62.7\left(c=0.01, \mathrm{CHCl}_{3}\right) ; \operatorname{TLC}(\mathrm{EtOAc} / \mathrm{Hex}=20 / 80): \mathrm{R}_{f}=0.15$.

$$
\mathrm{HO}^{-\mathrm{NH}} \cdot \stackrel{\stackrel{\mathrm{NaHCO}_{3}(3.0 \text { equiv) }}{\mathrm{BnCO}_{2} \mathrm{Cl}(1.0 \text { equiv) }} \longrightarrow}{\longrightarrow} \mathrm{HO}^{-\mathrm{N}_{1}} \stackrel{\mathrm{CO}_{2} \mathrm{Bn}}{\mathrm{H}}
$$

Benzyl hydroxycarbamate: A 3-necked $2000 \mathrm{~mL}$ round-bottomed flask equipped with a mechanical stirring apparatus was charged with hydroxylamine hydrochloride $\left(23.7 \mathrm{~g}, 340 \mathrm{mmol}, 1.2\right.$ equiv), $\mathrm{CH}_{2} \mathrm{Cl}_{2}(770 \mathrm{~mL})$, water $(39 \mathrm{~mL})$, and $\mathrm{NaHCO}_{3}$ $(71.5 \mathrm{~g}, 851 \mathrm{mmol}, 3.0$ equiv). The suspension was stirred at $\mathrm{rt}$ for $1 \mathrm{~h}$ then $\mathrm{Cbz}-\mathrm{Cl}(40.0 \mathrm{~mL}, 284 \mathrm{mmol}, 1.0$ equiv) was added dropwise over $30 \mathrm{~min}$. After stirring for an additional $16 \mathrm{~h}$, the reaction was filtered through a fritted filter funnel and the filter cake was washed once with brine. The biphasic mixture was transferred to a separatory funnel and the organic layer was collected. The aqueous layer was extracted with EtOAc $(2 \times 50 \mathrm{~mL})$ and the combined organic extracts were dried over sodium sulfate, filtered, and concentrated in vacuo to afford a white powder. The crude product was washed with $\mathrm{CH}_{2} \mathrm{Cl}_{2} /$ hexanes $=20 / 80$ to remove residual benzyl alcohol, thereby providing the title compound as a white powder ( 41.8 g, $250 \mathrm{mmol}$, 88\%). The spectral data for this compound were in agreement with reported literature values. ${ }^{8}$

Analytical data: ${ }^{1} \mathbf{H}$ NMR $\left(400 \mathrm{MHz}, \mathrm{CDCl}_{3}\right) \delta 7.37-7.27(\mathrm{~m}, 5 \mathrm{H}), 5.15(\mathrm{~s}, 2 \mathrm{H})$. 


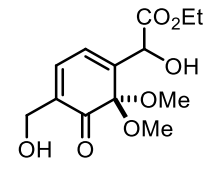

$( \pm)-4$
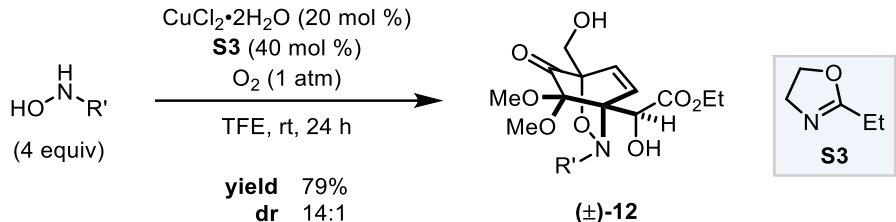

$( \pm)-12$

(士)-Benzyl azabicyclo[2.2.2]oct-5-ene-3-carboxylate (12): A $100 \mathrm{~mL}$ round-bottomed flask equipped with a stir bar was charged sequentially with dieneone ( \pm )-4 $(5.000 \mathrm{~g}, 17.47 \mathrm{mmol}, 1.0$ equiv), trifluoroethanol (35 mL), 2-ethyl-4,5-dihydrooxazole S3 ( $0.705 \mathrm{~mL}, 6.986 \mathrm{mmol}, 0.40$ equiv), and copper(ii) chloride dihydrate ( $595.5 \mathrm{mg}, 3.493 \mathrm{mmol}, 0.20$ equiv). The resulting dark green solution was sparged with a balloon of $\mathrm{O}_{2}$ for $5 \mathrm{~min}$ then benzyl hydroxycarbamate $(5.839 \mathrm{~g}, 34.93 \mathrm{~g}, 2.0$ equiv) was added in a single portion. After stirring at rt for $4 \mathrm{~h}$, more benzyl hydroxycarbamate $(3.750 \mathrm{~g}, 22.40 \mathrm{mmol}, 1.3 \mathrm{equiv})$ was added. The mixture was stirred at $\mathrm{rt}$ for an additional $20 \mathrm{~h}$ then poured onto Celite ${ }^{\circ}(\sim 10 \mathrm{~g})$ and filtered through a plug of Celite ${ }^{\circledR}$ with diethyl ether. The filtrate was concentrated in vacuo. NOTE: The water bath of a rotovap should be kept below $30{ }^{\circ} \mathrm{C}$ to prevent [4+2]-cycloreversion. The diastereomer ratio was determined by ${ }^{1} \mathrm{H}$ NMR spectroscopic analysis of the crude reaction mixture and purified material by comparison of the integration of the resonances at $\delta 6.40$ (minor diastereomer) and $\delta 6.35$ (major diastereomer). The crude material was purified by flash column chromatography (silica gel, diethyl ether/petroleum ethers $=50 / 50$ to $60 / 40$ to $70 / 30$ ) to afford the title compound as a yellow foam which becomes a yellow oil on standing $(6.20 \mathrm{~g}, 13.7 \mathrm{mmol}, 79 \%)$; the product was stored at $-20^{\circ} \mathrm{C}$ or used immediately.

Analytical data: ${ }^{1} \mathrm{H}$ NMR $\left(600 \mathrm{MHz}, \mathrm{CDCl}_{3}\right) \delta 7.39-7.32(\mathrm{~m}, 6 \mathrm{H}), 7.19(\mathrm{~d}, J=8.5 \mathrm{~Hz}, 1 \mathrm{H}), 6.41(\mathrm{~d}, J=8.5 \mathrm{~Hz}, 1 \mathrm{H}$, minor diastereomer), $6.35(\mathrm{~d}, J=8.6 \mathrm{~Hz}, 1 \mathrm{H}$, major diastereomer), $5.42(\mathrm{~d}, J=11.0 \mathrm{~Hz}, 1 \mathrm{H}), 5.23(\mathrm{~d}, J=12.0 \mathrm{~Hz}, 1 \mathrm{H}), 5.12(\mathrm{~d}, J$ $=12.0 \mathrm{~Hz}, 1 \mathrm{H}), 4.25(\mathrm{dq}, J=10.8,7.1 \mathrm{~Hz}, 1 \mathrm{H}), 4.19(\mathrm{~d}, J=11.1 \mathrm{~Hz}, 1 \mathrm{H}), 4.14(\mathrm{dq}, J=10.8,7.2 \mathrm{~Hz}, 1 \mathrm{H}), 4.03(\mathrm{dd}, J=13.3$, 10.3, $1 \mathrm{H}$ ), $3.96(\mathrm{dd}, J=13.3,3.7 \mathrm{~Hz}, 1 \mathrm{H}), 3.61(\mathrm{~s}, 3 \mathrm{H}), 3.35(\mathrm{~s}, 3 \mathrm{H}), 1.99(\mathrm{dd}, J=10.3,3.8 \mathrm{~Hz}, 1 \mathrm{H}), 1.29(\mathrm{t}, J=7.2 \mathrm{~Hz}, 3 \mathrm{H}$ ); ${ }^{13} \mathrm{C}\left\{{ }^{1} \mathrm{H}\right\}$ NMR $\left(151 \mathrm{MHz}, \mathrm{CDCl}_{3}\right) \delta 190.8,170.8,159.5,142.4,134.6,128.9,128.8,128.4,123.8,95.7,84.2,73.5,70.8$, 69.3, 61.4, 58.6, 55.8, 52.8, 14.1; HRMS (ESI+) m/z: [M + Na]+ Calcd. for $\mathrm{C}_{21} \mathrm{H}_{25} \mathrm{NO}_{10} \mathrm{Na}$ 474.1371; Found 474.1363; IR (thin film, $\mathrm{cm}^{-1}$ ): 3055, 2986, 2305, 1733, 1421, 1265, 896, 741, 705; TLC (diethyl ether/petroleum ethers $=60 / 40$ ): $R_{f}=0.10$.

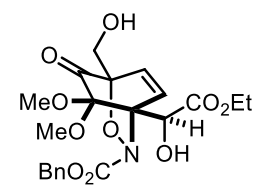

(士)-12

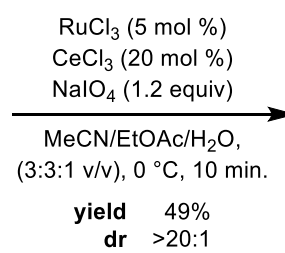

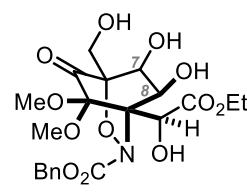

(士)-13

(士)-Benzyl 4-(-2-ethoxy-1-hydroxy-2-oxoethyl)-7,8-dihydroxy-1-(hydroxymethyl)-5,5-dimethoxy-6-oxo-2-oxa-3azabicyclo[2.2.2] octane-3-carboxylate (13): A $250 \mathrm{~mL}$ round-bottomed flask equipped with a stir bar was charged with sodium periodate $(3.525 \mathrm{~g}, 16.48 \mathrm{mmol}, 1.2$ equiv), cerium(iii) chloride heptahydrate $(1.023 \mathrm{~g}, 2.747 \mathrm{mmol}, 0.20$ equiv), and water $(7.1 \mathrm{~mL})$. No attempt was made to exclude air or moisture. The mixture was stirred at rt until a yellow precipitate was observed (typically $5 \mathrm{~min})$. The suspension was cooled to $0^{\circ} \mathrm{C}$ and charged with EtOAc $(21 \mathrm{~mL})$ and MeCN $(42 \mathrm{~mL})$. After stirring for $5 \mathrm{~min}$, a solution of ruthenium(iii) chloride dihydrate $(0.1 \mathrm{M}$ in water, $6.9 \mathrm{~mL}, 0.6867 \mathrm{mmol}, 0.05$ equiv) was added and the resulting mixture was stirred for $10 \mathrm{~min}$. A solution of cycloadduct 12 (6.20 g, $13.7 \mathrm{mmol}, 1.0 \mathrm{equiv})$ in EtOAc (21 $\mathrm{mL}$ ) was added and the dark brown mixture was stirred for 10 min. The reaction was quenched with saturated aqueous sodium thiosulfate $(50 \mathrm{~mL})$ and the aqueous layer was extracted with EtOAc $(3 \times 100 \mathrm{~mL})$. The combined organic extracts were washed with brine, dried over sodium sulfate, filtered, and concentrated in vacuo to afford a white foam. ${ }^{1} \mathrm{H}$ NMR analysis indicated that the crude material consisted of a $\sim 1: 1$ mixture of starting material and desired product. The crude material was then resubjected to the conditions described above to afford a white foam. The crude material was purified by flash column chromatography (silica gel, EtOAc/Hex $=50 / 50$ to $60 / 40$ to $80 / 20$ ) to provide the title compound as a white foam $(3.28 \mathrm{~g}, 6.76 \mathrm{mmol}, 49 \%)$.

Analytical data: $\mathbf{m p} 81-85^{\circ} \mathrm{C} ;{ }^{1} \mathrm{H}$ NMR $\left(600 \mathrm{MHz}, \mathrm{CDCl}_{3}\right) \delta 7.40-7.30(\mathrm{~m}, 5 \mathrm{H}), 5.45(\mathrm{~d}, J=11.1 \mathrm{~Hz}, 1 \mathrm{H}), 5.25(\mathrm{~d}, J=12.1$ $\mathrm{Hz}, 1 \mathrm{H}), 5.17(\mathrm{~d}, J=12.1 \mathrm{~Hz}, 1 \mathrm{H}), 4.95(\mathrm{~d}, J=11.1 \mathrm{~Hz}, 1 \mathrm{H}), 4.69(\mathrm{~d}, J=7.2 \mathrm{~Hz}, 1 \mathrm{H}), 4.47(\mathrm{~d}, J=7.0 \mathrm{~Hz}, 1 \mathrm{H}), 4.35-4.30$ $(\mathrm{m}, 1 \mathrm{H}), 4.23(\mathrm{q}, J=7.1 \mathrm{~Hz}, 2 \mathrm{H}), 4.04(\mathrm{~d}, J=13.2 \mathrm{~Hz}, 1 \mathrm{H}), 3.90(\mathrm{~d}, J=13.1 \mathrm{~Hz}, 1 \mathrm{H}), 3.76(\mathrm{~d}, J=5.9 \mathrm{~Hz}, 1 \mathrm{H}), 3.57(\mathrm{~s}, 3 \mathrm{H})$, $3.53(\mathrm{~s}, 3 \mathrm{H}), 1.27(\mathrm{t}, J=7.1 \mathrm{~Hz}, 3 \mathrm{H}) ;{ }^{13} \mathrm{C}\left\{{ }^{1} \mathrm{H}\right\}$ NMR $\left(151 \mathrm{MHz}, \mathrm{CDCl}_{3}\right) \delta 199.2,172.1,157.0,134.9,128.7,128.7,128.3$, 128.3, 99.9, 83.9, 71.2, 70.0, 69.1, 66.8, 61.8, 60.4, 58.8, 55.5, 52.9, 14.1; HRMS (ESI+) m/z: [M + Na] ${ }^{+}$Calcd. for $\mathrm{C}_{21} \mathrm{H}_{27} \mathrm{NO}_{12} \mathrm{Na}$ 508.1431; Found 508.1416; IR (thin film, $\mathrm{cm}^{-1}$ ): 3468, 2956, 2924, 2853, 2359, 1808, 1736, 1457, 1378, $1273,1210,1098,1015,847,740699 ;$ TLC $(E t O A c / H e x=70 / 30): R_{f}=0.32$. 


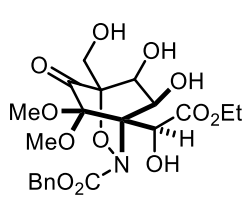

$( \pm)-13$

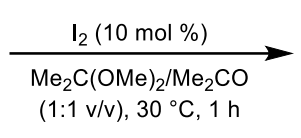

yield $74 \%$

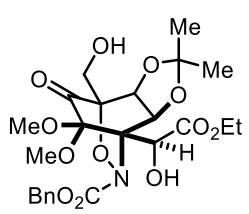

(士)-S4

(士)-Benzyl 7-(2-ethoxy-1-hydroxy-2-oxoethyl)-4-(hydroxymethyl)-6,6-dimethoxy-2,2-dimethyl-5-oxohexahydro-4,7(epoxyimino)benzo[d][1,3]dioxole-8-carboxylate (S4): A scintillation vial equipped with a stir bar was charged with tetrol 13 (750.0 mg, $1.545 \mathrm{mmol}, 1.0$ equiv), acetone (3.9 mL), 2,2-dimethoxypropane (3.9 mL), and $\mathrm{I}_{2}(39.2 \mathrm{mg}, 0.155 \mathrm{mmol}$, 0.10 equiv). No attempt was made to exclude air or moisture. The solution was stirred at $30^{\circ} \mathrm{C}$ for $1 \mathrm{~h}$ then cooled to rt and quenched with saturated aqueous sodium thiosulfate. The aqueous phase was extracted with EtOAc $(3 \times 10 \mathrm{~mL})$ and the combined organic extracts were washed with brine, dried over sodium sulfate, filtered, and concentrated in vacuo to provide a white foam. The crude material was purified by flash column chromatography (silica gel, EtOAc/Hex $=30 / 70$ to $40 / 60$ ) to provide the title compound as a white powder (601.6 $\mathrm{mg}, 1.145 \mathrm{mmol}, 74 \%)$.

Analytical data: $\mathbf{m p ~} 66-71^{\circ} \mathrm{C} ;{ }^{1} \mathrm{H}$ NMR $\left(600 \mathrm{MHz}, \mathrm{CDCl}_{3}\right) \delta 7.43-7.32(\mathrm{~m}, 5 \mathrm{H}), 5.55$ (dd, $\left.J=10.7,4.2 \mathrm{~Hz}, 1 \mathrm{H}\right), 5.34(\mathrm{~d}, J$ $=12.0 \mathrm{~Hz}, 1 \mathrm{H}), 5.17(\mathrm{~d}, J=12.0 \mathrm{~Hz}, 1 \mathrm{H}), 5.14(\mathrm{~d}, J=10.8 \mathrm{~Hz}, 1 \mathrm{H}), 5.05(\mathrm{~d}, J=7.1 \mathrm{~Hz}, 1 \mathrm{H}), 4.46(\mathrm{~d}, J=7.2 \mathrm{~Hz}, 1 \mathrm{H}), 4.17$ (qd, $J=7.2,1.4 \mathrm{~Hz}, 2 \mathrm{H}), 4.00(\mathrm{dd}, J=13.4,9.7 \mathrm{~Hz}, 1 \mathrm{H}), 3.87(\mathrm{dd}, J=13.4,3.7 \mathrm{~Hz}, 1 \mathrm{H}), 3.67(\mathrm{~s}, 3 \mathrm{H}), 3.48(\mathrm{~s}, 3 \mathrm{H}), 1.80(\mathrm{dd}$, $J=9.9,4.2 \mathrm{~Hz}, 1 \mathrm{H}), 1.35(\mathrm{~s}, 3 \mathrm{H}), 1.28(\mathrm{~s}, 3 \mathrm{H}), 1.27(\mathrm{t}, J=7.2 \mathrm{~Hz}, 3 \mathrm{H}) ;{ }^{13} \mathrm{C}\left\{{ }^{1} \mathrm{H}\right\} \mathbf{N M R}\left(151 \mathrm{MHz}, \mathrm{CDCl}_{3}\right) \delta$ 198.7, 170.0, 157.6, 134.9, 128.9, 128.8, 128.6, 111.6, 98.2, 84.1, 72.6, 71.9, 71.0, 69.2, 69.1, 60.9, 58.1, 55.8, 53.2, 25.3, 24.1, 14.0; HRMS (ESI $\left.{ }^{+}\right) \mathrm{m} / \mathrm{z}:[\mathrm{M}+\mathrm{H}]^{+}$Calcd. for $\mathrm{C}_{24} \mathrm{H}_{32} \mathrm{NO}_{12} \mathrm{H}$ 526.1919; Found: 526.1914; TLC $(\mathrm{EtOAc} / \mathrm{Hex}=40 / 60): \mathrm{R}_{f}=0.27$.

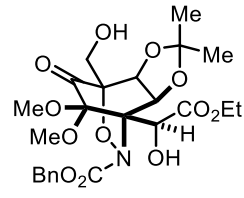

(士)-S4

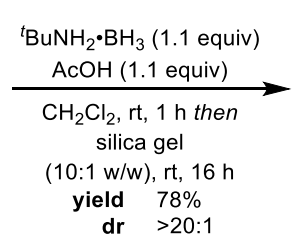

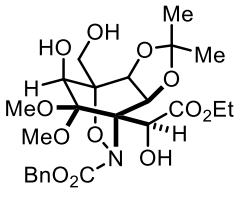

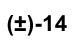

( \pm )-Benzyl-7-(2-ethoxy-1-hydroxy-2-oxoethyl)-5-hydroxy-4-(hydroxymethyl)-6,6-dimethoxy-2,2-dimethylhexahydro4,7-(epoxyimino)benzo[d][1,3]dioxole-8-carboxylate (14): A scintillation vial equipped with a stir bar was charged with acetonide S4 (447.1 mg, $0.8508 \mathrm{mmol}, 1.0$ equiv), $\mathrm{CH}_{2} \mathrm{Cl}_{2}(6.0 \mathrm{~mL}), \mathrm{AcOH}$ (53.57 $\mu \mathrm{L}, 0.9359 \mathrm{mmol}, 1.1$ equiv), and ${ }^{t} \mathrm{BuNH}_{2} \cdot \mathrm{BH}_{3}(81.4 \mathrm{mg}, 0.936 \mathrm{mmol}, 1.1$ equiv). No attempt was made to exclude air or moisture. The reaction was stirred at $\mathrm{rt}$ for $1 \mathrm{~h}$ then silica gel $(4.5 \mathrm{~g})$ was added in one portion. The suspension was stirred at $\mathrm{rt}$ for $16 \mathrm{~h}$ then filtered through a plug of Celite $\AA$ with $10 \% \mathrm{MeOH} / \mathrm{CH}_{2} \mathrm{Cl}_{2}(100 \mathrm{~mL})$ and the combined filtrate was concentrated in vacuo to provide a white foam. The crude material was purified by flash column chromatography (silica gel, EtOAc/Hex $=60 / 40$ to $70 / 30$ ) to afford the title compound as a white powder (349.1 $\mathrm{mg}, 0.6618 \mathrm{mmol}, 78 \%)$.

Analytical data: mp $51-55^{\circ} \mathrm{C} ;{ }^{1} \mathrm{H}$ NMR $\left(600 \mathrm{MHz}, \mathrm{CDCl}_{3}\right) \delta 7.40-7.30(\mathrm{~m}, 5 \mathrm{H}), 5.45(\mathrm{~s}, 1 \mathrm{H}), 5.21(\mathrm{~d}, \mathrm{~J}=12.1 \mathrm{~Hz}, 1 \mathrm{H})$, $5.16(\mathrm{~d}, J=12.2 \mathrm{~Hz}, 1 \mathrm{H}), 4.62(\mathrm{~d}, J=8.1 \mathrm{~Hz}, 1 \mathrm{H}), 4.33(\mathrm{dd}, J=8.2,1.6 \mathrm{~Hz}, 1 \mathrm{H}), 4.28-4.14(\mathrm{~m}, 2 \mathrm{H}), 4.08(\mathrm{~d}, J=7.7 \mathrm{~Hz}$, $1 \mathrm{H}), 4.01(\mathrm{~d}, J=12.5 \mathrm{~Hz}, 1 \mathrm{H}), 3.75(\mathrm{~d}, J=12.5 \mathrm{~Hz}, 1 \mathrm{H}), 3.57(\mathrm{~s}, 3 \mathrm{H}), 3.29(\mathrm{~s}, 3 \mathrm{H}), 3.02(\mathrm{~d}, J=10.6 \mathrm{~Hz}, 1 \mathrm{H}), 1.53(\mathrm{~s}, 3 \mathrm{H})$, $1.31-1.30(\mathrm{~m}, 6 \mathrm{H}) ;{ }^{13} \mathrm{C}\left\{{ }^{1} \mathrm{H}\right\}$ NMR $\left(151 \mathrm{MHz}, \mathrm{CDCl}_{3}\right) \delta 167.2,154.1,134.7,128.6,128.6,128.5,128.3,111.1,98.4,75.1$, 73.1, 73.0, 72.9, 72.8, 70.4, 63.7, 63.7, 61.9, 51.3, 48.6, 24.1, 23.8, 14.0; HRMS (ESI+) m/z: [M + H] ${ }^{+}$Calcd. for $\left[\mathrm{C}_{24} \mathrm{H}_{34} \mathrm{NO}_{12}\right.$ 528.2081; Found 528.2074; IR (thin film, $\mathrm{cm}^{-1}$ ): 3545, 2983, 2945, 1751, 1456, 1383, 1268, 1208, 1135, 1064, 736; TLC $(\mathrm{EtOAc} / \mathrm{Hex}=70 / 30): \mathrm{R}_{f}=0.17$.

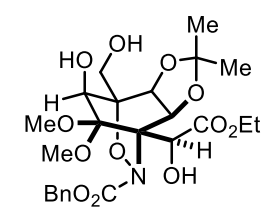

(士)-14

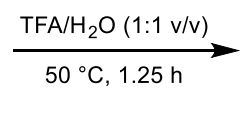

yield $44 \%$

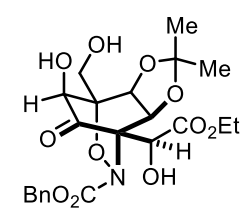

(士)-15

(士)-Benzyl 7-(2-ethoxy-1-hydroxy-2-oxoethyl)-5-hydroxy-4-(hydroxymethyl)-2,2-dimethyl-6-oxohexahydro-4,7(epoxyimino)benzo[d][1,3]dioxole-8-carboxylate (15): A scintillation vial was charged with bisketal 14 (200.0 mg, 0.3791 mmol, 1.0 equiv), water $(1.26 \mathrm{~mL})$, and TFA $(1.26 \mathrm{~mL})$. The solution was stirred at $50{ }^{\circ} \mathrm{C}$ for $1.25 \mathrm{~h}$, cooled to rt, and concentrated in vacuo. Toluene $(2 \mathrm{~mL})$ was added to the crude residue and concentrated in vacuo three times to remove 
traces of TFA. The crude material was purified by flash column chromatography (silica gel, EtOAc/Hex $=60 / 40$ ) to afford the title compound as a white powder $(81.0 \mathrm{mg}, 0.168 \mathrm{mmol}, 44 \%)$.

Analytical data: $\mathbf{m p} 95-99{ }^{\circ} \mathrm{C} ;{ }^{1} \mathbf{H}$ NMR $\left(600 \mathrm{MHz}, \mathrm{CDCl}_{3}\right) \delta 7.43-7.30(\mathrm{~m}, 5 \mathrm{H}), 5.99(\mathrm{~s}, 1 \mathrm{H}), 5.27(\mathrm{~d}, \mathrm{~J}=12.2 \mathrm{~Hz}, 1 \mathrm{H})$, $5.21(\mathrm{~d}, J=12.2 \mathrm{~Hz}, 1 \mathrm{H}), 4.80(\mathrm{~s}, 1 \mathrm{H}), 4.70(\mathrm{~d}, J=6.7 \mathrm{~Hz}, 1 \mathrm{H}), 4.46(\mathrm{~d}, J=6.8 \mathrm{~Hz}, 1 \mathrm{H}), 4.30-4.12(\mathrm{~m}, 2 \mathrm{H}), 4.09(\mathrm{~d}, J=$ $13.0 \mathrm{~Hz}, 1 \mathrm{H}), 3.83(\mathrm{~d}, J=12.9 \mathrm{~Hz}, 1 \mathrm{H}), 1.34(\mathrm{~s}, 3 \mathrm{H}), 1.26(\mathrm{~s}, 3 \mathrm{H}), 1.17(\mathrm{t}, J=7.1 \mathrm{~Hz}, 3 \mathrm{H}) ;{ }^{13} \mathrm{C}\left\{{ }^{1} \mathrm{H}\right\} \mathbf{N M R}(151 \mathrm{MHz}, \mathrm{CDCl} 3)$ б 204.8, 166.8, 153.8, 134.7, 128.7, 128.6, 128.2, 112.0, 80.5, 77.1, 74.5, 73.9, 70.7, 70.5, 68.2, 62.7, 62.3, 25.1, 24.2, 13.7; HRMS (ESI $\left.{ }^{+}\right) \mathrm{m} / \mathrm{z}:[\mathrm{M}+\mathrm{Na}]^{+}$Calcd. for $\mathrm{C}_{22} \mathrm{H}_{27} \mathrm{NO}_{11} \mathrm{Na}$ 504.1482; Found: 504.1463; TLC $(\mathrm{EtOAc} / \mathrm{Hex}=60 / 40): \mathrm{R}_{f}=$ 0.18 .

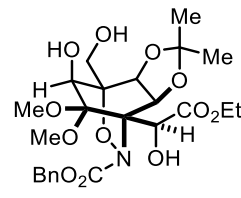

$( \pm)-14$

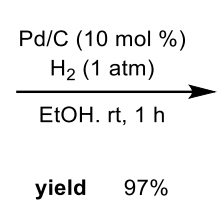

yield $97 \%$

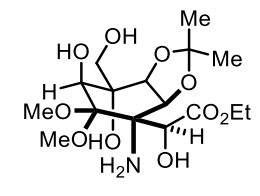

(士)-S5

(士)-Ethyl 4-amino-6,7-dihydroxy-7-(hydroxymethyl)-5,5-dimethoxy-2,2-dimethylhexahydrobenzo[d][1,3]dioxol-4yl)-2-hydroxyacetate (S5): A scintillation vial equipped with a stir bar was charged with triol $14(150.9 \mathrm{mg}, 0.2861 \mathrm{mmol}$, 1.0 equiv), $\mathrm{EtOH}(2.8 \mathrm{~mL})$, and palladium on charcoal $(10 \% \mathrm{w} / \mathrm{w}, 30.4 \mathrm{mg}, 0.0286 \mathrm{mmol}, 0.10$ equiv). The vessel was sealed with a rubber septum and the mixture was sparged with a balloon of hydrogen gas for $10 \mathrm{~min}$. After stirring for an additional $50 \mathrm{~min}$, the reaction mixture was filtered through a pad of Celite ${ }^{\circledR}$ and the filtrate was concentrated in vacuo to provide the title compound as a white foam (109.6 mg, $0.2772 \mathrm{mmol}, 97 \%)$. No further purification was required.

Analytical data: $\mathbf{m p} 145-147^{\circ} \mathrm{C} ;{ }^{1} \mathrm{H}$ NMR $\left(600 \mathrm{MHz}, \mathrm{CDCl}_{3}\right) \delta 4.74(\mathrm{~s}, 1 \mathrm{H}), 4.48(\mathrm{dd}, J=7.4,1.4 \mathrm{~Hz}, 1 \mathrm{H}), 4.28(\mathrm{dq}, J=$ 11.0, 7.2 Hz, 1H), $4.18(\mathrm{~d}, J=7.4 \mathrm{~Hz}, 1 \mathrm{H}), 4.17(\mathrm{dq}, J=11.0,7.2 \mathrm{~Hz}, 1 \mathrm{H}), 4.06(\mathrm{dd}, J=11.2,1.3 \mathrm{~Hz}, 1 \mathrm{H}), 3.97(\mathrm{~d}, J=11.4$ $\mathrm{Hz}, 1 \mathrm{H}), 3.75(\mathrm{~d}, J=11.4 \mathrm{~Hz}, 1 \mathrm{H}), 3.55(\mathrm{~s}, 3 \mathrm{H}), 3.44(\mathrm{~s}, 3 \mathrm{H}), 3.18(\mathrm{~d}, J=11.2), 2.91(\mathrm{br} \mathrm{s}, 1 \mathrm{H}), 1.72(\mathrm{br} \mathrm{s}, 1 \mathrm{H}), 1.46(\mathrm{~s}, 3 \mathrm{H})$, $1.33(\mathrm{t}, J=7.2 \mathrm{~Hz}, 3 \mathrm{H}), 1.31(\mathrm{~s}, 3 \mathrm{H}) ;{ }^{13} \mathrm{C}\left\{{ }^{1} \mathrm{H}\right\}$ NMR $\left(151 \mathrm{MHz}, \mathrm{CDCl}_{3}\right) \delta 172.8,109.6,101.7,76.7,76.4,76.3,72.7,70.1$, 65.7, 63.7, 61.3, 52.4, 25.3, 24.2, 14.3; HRMS (ESI+) m/z: [M + H]+ Calcd. for $\mathrm{C}_{16} \mathrm{H}_{30} \mathrm{NO}_{10}$ 396.1869; Found: 396.1870; IR (thin film, $\left.\mathrm{cm}^{-1}\right)$ : 3369, 2982, 2926, 2848, 1733, 1381, 1261, 1209, 1073, 880; TLC (EtOAc/Hex = 70/30): $\mathrm{R}_{f}=0.15$.

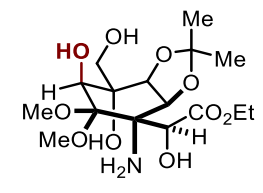

(士)-S5

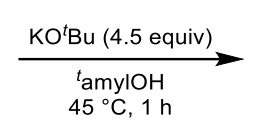

yield $68 \%$

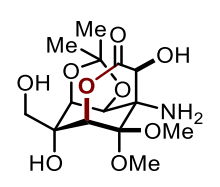

$( \pm)-17$

(士)-9-Amino-4,8-dihydroxy-4-(hydroxymethyl)-10,10-dimethoxy-2,2-dimethylhexahydro-7H-5,9-

methano[1,3]dioxolo[4,5-d]oxocin-7-one (17): A 1-dram vial equipped with a stir flea was charged with aminoalcohol S5 (25.0 mg, $0.0632 \mathrm{mmol}, 1.0$ equiv), tamylOH (1.26 mL), and KOtBu (31.9 mg, $0.285 \mathrm{mmol}, 4.5$ equiv). The resulting yellow solution was stirred at $\mathrm{rt}$ for $1 \mathrm{~h}$ and diluted with saturated aqueous ammonium chloride and brine solutions ( 1:1 v/v). The aqueous phase was extracted with EtOAc $(3 \times 5 \mathrm{~mL})$ and the combined organic extracts were dried over sodium sulfate, filtered, and concentrated in vacuo to provide a white foam. The crude material was purified by flash column chromatography (silica gel, $\mathrm{MeOH} / \mathrm{CH}_{2} \mathrm{Cl}_{2}=0 / 100$ to $1 / 99$ to $2.5 / 97.5$ ) to provide the title compound as a white powder (14.9 mg, 0.0427 $\mathrm{mmol}, 68 \%)$.

Analytical data: $\mathbf{m p} 135-139{ }^{\circ} \mathrm{C}$ (decomp.); ${ }^{1} \mathrm{H}$ NMR $\left(600 \mathrm{MHz}, \mathrm{CDCl}_{3}\right) \delta 4.68(\mathrm{~d}, J=1.3 \mathrm{~Hz}, 1 \mathrm{H}), 4.65(\mathrm{~d}, J=1.9 \mathrm{~Hz}, 1 \mathrm{H})$, $4.47(\mathrm{~d}, J=5.2 \mathrm{~Hz}, 1 \mathrm{H}), 4.36(\mathrm{dd}, J=5.2,1.3 \mathrm{~Hz}, 1 \mathrm{H}), 4.08(\mathrm{dd}, J=12.4,5.4 \mathrm{~Hz}, 1 \mathrm{H}), 3.82(\mathrm{~d}, J=11.9 \mathrm{~Hz}, 1 \mathrm{H}), 3.78(\mathrm{~s}$, $3 \mathrm{H}), 3.40(\mathrm{~s}, 3 \mathrm{H}), 2.27-2.20(\mathrm{~m}, 1 \mathrm{H}), 2.00(\mathrm{~s}, 2 \mathrm{H}), 1.59(\mathrm{~s}, 3 \mathrm{H}), 1.31-1.27(\mathrm{~m}, 3 \mathrm{H}), 1.22(\mathrm{~s}, 3 \mathrm{H}) ;{ }^{13} \mathrm{C}\left\{{ }^{1} \mathrm{H}\right\} \mathrm{NMR}(151 \mathrm{MHz}$, $\left.\left.\mathrm{CDCl}_{3}\right) \delta 188.9,153.9,110.9,99.8,79.7,77.4,72.7,72.7,71.8,63.9,54.3,49.7,29.7,25.2,25.2 ; \mathbf{H R M S}_{(\mathbf{E S I}}{ }^{+}\right) \mathrm{m} / \mathrm{z}:[\mathrm{M}+$ $\mathrm{H}]^{+}$Calcd. for $\mathrm{C}_{14} \mathrm{H}_{22} \mathrm{NO}_{9} 348.1294$; Found: 348.1283; TLC $\left(\mathrm{MeOH} / \mathrm{CH}_{2} \mathrm{Cl}_{2}=5 / 95\right): \mathrm{R}_{f}=0.15$. 


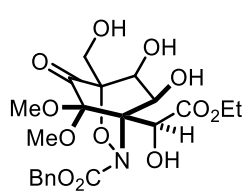

$( \pm)-13$

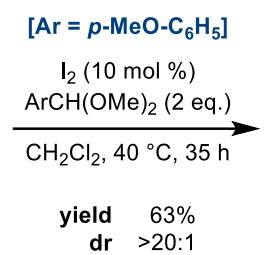

dr $>20: 1$

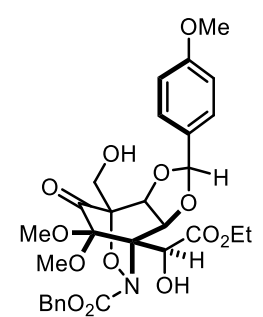

(士)-S6

(士)-Benzyl 7-(2-ethoxy-1-hydroxy-2-oxoethyl)-4-(hydroxymethyl)-6,6-dimethoxy-2-(4-methoxyphenyl)-5oxohexahydro-4,7-(epoxyimino)benzo[d][1,3]dioxole-8-carboxylate (S6): A flame-dried scintillation vial equipped with a magnetic stir bar was cooled under a stream of $\mathrm{N}_{2}$ and charged with tetrol 13 (1.000 g, $2.060 \mathrm{mmol}, 1.0$ equiv), dry $\mathrm{CH}_{2} \mathrm{Cl}_{2}$ $(5.15 \mathrm{~mL}), \mathrm{I}_{2}$ (52.3 mg, $0.206 \mathrm{mmol}, 0.1$ equiv), and $p$-anisaldehyde dimethyl acetal $(0.70 \mathrm{~mL}, 4.12 \mathrm{mmol}, 2.0$ equiv). The dark purple solution was stirred at $40{ }^{\circ} \mathrm{C}$ for $3 \mathrm{~h}$ then cooled to rt and diluted with saturated aqueous sodium thiosulfate. The aqueous layer was extracted with $\mathrm{CH}_{2} \mathrm{Cl}_{2}(3 \times 10 \mathrm{~mL})$ and the combined organic extracts were dried over sodium sulfate, filtered, and concentrated in vacuo. The crude material was purified by flash column chromatography (silica gel, EtOAc/Hex $=30 / 70$ to $40 / 60$ ) to provide the PMP ketal $( \pm)-S 6$ as a white foam (784.3 $\mathrm{mg}, 1.299 \mathrm{mmol}, 63 \%)$.

Analytical data: mp 87-91 ${ }^{\circ} \mathrm{C} ;{ }^{1} \mathrm{H}$ NMR $\left(600 \mathrm{MHz}, \mathrm{CDCl}_{3}\right) \delta 7.45-7.36(\mathrm{~m}, 5 \mathrm{H}), 7.29(\mathrm{~d}, \mathrm{~J}=8.8 \mathrm{~Hz}, 2 \mathrm{H}), 6.87(\mathrm{~d}, \mathrm{~J}=8.7 \mathrm{~Hz}$, $2 \mathrm{H}), 5.64(\mathrm{~s}, 1 \mathrm{H}), 5.56(\mathrm{~d}, J=10.9 \mathrm{~Hz}, 1 \mathrm{H}), 5.36(\mathrm{~d}, J=12.0 \mathrm{~Hz}, 1 \mathrm{H}), 5.20(\mathrm{~d}, J=10.8 \mathrm{~Hz}, 1 \mathrm{H}), 5.19(J=12.0 \mathrm{~Hz}, 1 \mathrm{H}), 5.12$ (d, $J=7.6 \mathrm{~Hz}, 1 \mathrm{H}$ ), $4.55(\mathrm{~d}, J=7.5 \mathrm{~Hz}, 1 \mathrm{H}), 4.17$ (qd, $J=7.1,5.0 \mathrm{~Hz}, 2 \mathrm{H}), 4.07$ (dd, $J=13.4,9.9 \mathrm{~Hz}, 1 \mathrm{H}), 3.93$ (dd, $J=$ 13.4, $4.2 \mathrm{~Hz}, 1 \mathrm{H}), 3.81(\mathrm{~s}, 3 \mathrm{H}), 3.60(\mathrm{~s}, 3 \mathrm{H}), 3.49(\mathrm{~s}, 3 \mathrm{H}), 1.73(\mathrm{dd}, J=9.9,4.3 \mathrm{~Hz}, 1 \mathrm{H}), 1.24(\mathrm{t}, J=7.1 \mathrm{~Hz}, 3 \mathrm{H}) ;{ }^{13} \mathrm{C}\left\{{ }^{1} \mathrm{H}\right\}$ NMR $\left(151 \mathrm{MHz}, \mathrm{CDCl}_{3}\right) \delta 198.6,169.9,160.9,157.6,134.9,128.9,128.8,128.7,128.5,126.1,113.7,104.8,98.3,84.1$, 72.4, 72.0, 71.7, 69.3, 69.1, 60.9, 58.1, 55.6, 55.3, 53.6, 14.0; HRMS (ESI+) m/z: [M + H] ${ }^{+}$Calcd. for $\mathrm{C}_{29} \mathrm{H}_{34} \mathrm{NO}_{13}$ 604.2029; Found: 604.2016; IR (thin film, $\mathrm{cm}^{-1}$ ): 3502, 2951, 1811, 1746, 1615, 1520, 1456, 1398, 1308, 1253, 1074, 833, 737, 700, 597; TLC (EtOAc/Hex $=40 / 60): R_{f}=0.19$.

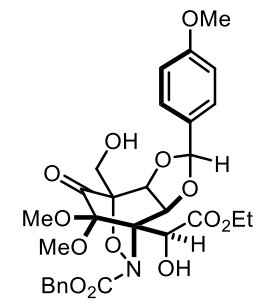

(士)-S6
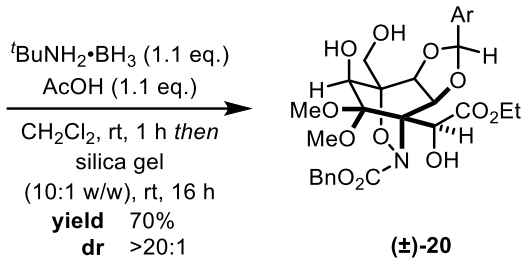

$( \pm)-20$

(士)-Benzyl

7-(2-ethoxy-1-hydroxy-2-oxoethyl)-5-hydroxy-4-(hydroxymethyl)-6,6-dimethoxy-2-(4methoxyphenyl)hexahydro-4,7-(epoxyimino)benzo[d][1,3]dioxole-8-carboxylate (20): A scintillation vial equipped with a stir bar was charged with PMP acetal $\mathbf{S 6}\left(480.0 \mathrm{mg}, 0.7950 \mathrm{mmol}, 1.0\right.$ equiv), $\mathrm{CH}_{2} \mathrm{Cl}_{2}(7.9 \mathrm{~mL}), \mathrm{AcOH}(50.1 \mu \mathrm{L}, 0.875$ mmol, 1.1 equiv), and ${ }^{t} \mathrm{BuNH}_{2} \cdot \mathrm{BH}_{3}(76.1 \mathrm{mg}, 0.875 \mathrm{mmol}, 1.1$ equiv). No attempt was made to exclude air or moisture. The reaction was stirred at rt for $2 \mathrm{~h}$ then silica gel $(4.5 \mathrm{~g})$ was added in one portion. The suspension was stirred at $\mathrm{rt}$ for $16 \mathrm{~h}$ then filtered through a short Celite ${ }^{\circledR}$ plug with $10 \% \mathrm{MeOH} / \mathrm{CH}_{2} \mathrm{Cl}_{2}(100 \mathrm{~mL})$ and the filtrate was concentrated in vacuo to provide a white foam. The crude material was purified by flash column chromatography (silica gel, EtOAc/ $\mathrm{Hex}=50 / 50$ to $60 / 40$ to $70 / 30$ ) to afford the title compound as a white powder ( $337.6 \mathrm{mg}, 0.5575 \mathrm{mmol}, 70 \%$ ).

Analytical data: $\mathbf{m p} 99-106{ }^{\circ} \mathrm{C} ;{ }^{1} \mathbf{H}$ NMR $\left(600 \mathrm{MHz}, \mathrm{CDCl}_{3}\right) \delta 7.45-7.31(\mathrm{~m}, 7 \mathrm{H}), 6.94-6.87(\mathrm{~m}, 2 \mathrm{H}), 6.28(\mathrm{~s}, 1 \mathrm{H}), 5.76$ $(\mathrm{s}, 1 \mathrm{H}), 5.46(\mathrm{~s}, 1 \mathrm{H}), 5.22(\mathrm{~d}, J=12.1 \mathrm{~Hz}, 1 \mathrm{H}), 5.17(\mathrm{~d}, J=12.1 \mathrm{~Hz}, 1 \mathrm{H}), 4.74(\mathrm{~d}, J=8.4 \mathrm{~Hz}, 1 \mathrm{H}), 4.39(\mathrm{dd}, J=8.4,1.6 \mathrm{~Hz}$, $1 \mathrm{H}), 4.27-4.06(\mathrm{~m}, 4 \mathrm{H}), 3.83(\mathrm{~s}, 3 \mathrm{H}), 3.25(\mathrm{~s}, 3 \mathrm{H}), 3.15(\mathrm{~d}, J=10.9 \mathrm{~Hz}, 1 \mathrm{H}), 3.12(\mathrm{~s}, 3 \mathrm{H}), 1.27(\mathrm{t}, J=7.2 \mathrm{~Hz}, 4 \mathrm{H}) ;{ }^{13} \mathrm{C}\left\{{ }^{1} \mathrm{H}\right\}$ NMR $\left(151 \mathrm{MHz}, \mathrm{CDCl}_{3}\right) \delta 167.1,160.1,154.1,134.6,128.6,128.5,128.3,127.6,127.5,113.5,104.0,98.0,75.5,74.3$, 73.2, 72.9, 72.8, 70.4, 63.7, 63.6, 61.6, 55.3, 51.0, 48.8, 13.9; HRMS (ESI+) m/z: [M + H] ${ }^{+}$Calcd. for $\mathrm{C}_{29} \mathrm{H}_{36} \mathrm{NO}_{13}$ 606.2186; Found 606.2169; IR (thin film, $\left.\mathrm{cm}^{-1}\right)$ : 3255, 2926, 2842, 2359, 1749, 1520, 1457, 1254, 1066, 786; TLC (EtOAc/Hex = 60/40): $\mathrm{R}_{f}=0.15$. 
${ }^{1} \mathrm{H}$ NMR $\left(600 \mathrm{MHz}, \mathrm{C}_{6} \mathrm{D}_{6}\right) \delta 7.40-7.34(\mathrm{~m}, 2 \mathrm{H}), 7.09-7.06(\mathrm{~m}, 2 \mathrm{H}), 7.05-6.99(\mathrm{~m}, 3 \mathrm{H}), 6.70(\mathrm{~d}, J=8.7 \mathrm{~Hz}, 2 \mathrm{H}), 6.47(\mathrm{~s}$, $1 \mathrm{H}), 5.88(\mathrm{~s}, 1 \mathrm{H}), 5.36(\mathrm{~s}, 1 \mathrm{H}), 4.23(\mathrm{~d}, J=12.5 \mathrm{~Hz}, 1 \mathrm{H}), 4.18(\mathrm{~d}, J=10.2 \mathrm{~Hz}, 1 \mathrm{H}), 4.14(\mathrm{dd}, J=8.4,1.6 \mathrm{~Hz}, 1 \mathrm{H}), 4.05(\mathrm{dq}$, $J=10.9,7.1 \mathrm{~Hz}, 1 \mathrm{H}), 3.97(\mathrm{dq}, J=10.9,7.2 \mathrm{~Hz}, 1 \mathrm{H}), 3.92(\mathrm{~d}, J=12.5 \mathrm{~Hz}, 1 \mathrm{H}), 3.29(\mathrm{~s}, 3 \mathrm{H}), 3.05(\mathrm{~s}, 3 \mathrm{H}), 2.93(\mathrm{~s}, 3 \mathrm{H}), 0.96$ $(\mathrm{t}, J=7.1 \mathrm{~Hz}, 3 \mathrm{H}) . ;{ }^{13} \mathrm{C}\left\{{ }^{1} \mathrm{H}\right\}$ NMR $\left(151 \mathrm{MHz}, \mathrm{C}_{6} \mathrm{D}_{6}\right) \delta 167.6,160.5,155.0,135.4,128.7,128.6,128.6,128.5,128.4,113.8$, $104.1,98.7,76.1,74.9,74.0,73.6,73.0,70.4,64.3,63.8,61.5,54.8,50.9,48.6,14.0$.
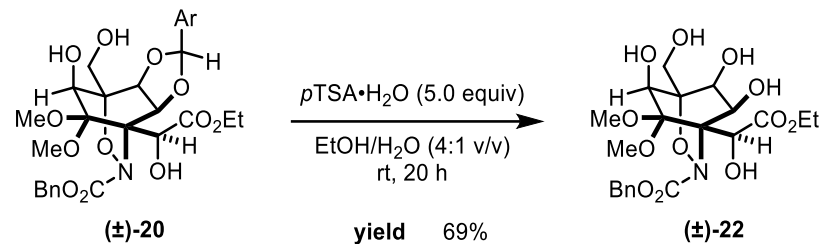

$( \pm)-22$

(士)-Benzyl 4-(2-ethoxy-1-hydroxy-2-oxoethyl)-6,7,8-trihydroxy-1-(hydroxymethyl)-5,5-dimethoxy-2-oxa-3azabicyclo[2.2.2] octane-3-carboxylate (24): A 1-dram vial equipped with a magnetic stir bar was charged with bis(acetal) $20\left(100.0 \mathrm{mg}, 0.1651 \mathrm{mmol}, 1.0\right.$ equiv), $\mathrm{EtOH}(2.6 \mathrm{~mL})$, water $(0.66 \mathrm{~mL})$, and $p \mathrm{TSA} \cdot \mathrm{H}_{2} \mathrm{O}(157.0 \mathrm{mg}, 0.8256 \mathrm{mmol}, 5.0$ equiv). The solution was stirred $\mathrm{rt}$ for $20 \mathrm{~h}$ and diluted with saturated aqueous sodium bicarbonate. The aqueous phase was extracted with EtOAc $(3 \times 10 \mathrm{~mL})$ and the combined organic extracts were washed with brine, dried over sodium sulfate, filtered, and concentrated in vacuo to provide a white foam. The crude material was purified by flash column chromatography (silica gel, $\mathrm{MeOH} / \mathrm{CH}_{2} \mathrm{Cl}_{2}=5 / 95$ ) to provide the title compound as a white foam (55.9 mg, $0.115 \mathrm{mmol}, 69 \%$ ).

Analytical data: $\mathrm{mp} 125-131{ }^{\circ} \mathrm{C} ;{ }^{1} \mathrm{H}$ NMR $\left(400 \mathrm{MHz}, \mathrm{CDCl}_{3}\right) \delta 7.39-7.32(\mathrm{~m}, 5 \mathrm{H}), 6.20(\mathrm{~s}, 1 \mathrm{H}), 5.48(\mathrm{~s}, 1 \mathrm{H}), 5.20(\mathrm{~d}, J=$ $12.1 \mathrm{~Hz}, 1 \mathrm{H}), 5.16(\mathrm{~d}, J=12.0 \mathrm{~Hz}, 1 \mathrm{H}), 4.36(\mathrm{t}, J=8.4 \mathrm{~Hz}, 1 \mathrm{H}), 4.29-4.15(\mathrm{~m}, 3 \mathrm{H}), 4.12-4.05(\mathrm{~m}, 1 \mathrm{H}), 4.02(\mathrm{dd}, J=12.6$, $6.7 \mathrm{~Hz}, 1 \mathrm{H}), 3.76(\mathrm{dd}, J=12.6,5.9 \mathrm{~Hz}, 1 \mathrm{H}), 3.61(\mathrm{~s}, 3 \mathrm{H}), 3.39(\mathrm{~s}, 3 \mathrm{H}), 3.36(\mathrm{~d}, J=8.6 \mathrm{~Hz}, 1 \mathrm{H}), 3.27(\mathrm{~d}, J=10.1 \mathrm{~Hz}, 1 \mathrm{H})$, $3.20(\mathrm{~d}, J=5.3 \mathrm{~Hz}, 1 \mathrm{H}), 1.30(\mathrm{t}, J=7.2 \mathrm{~Hz}, 3 \mathrm{H}) ;{ }^{13} \mathrm{C}\left\{{ }^{1} \mathrm{H}\right\}$ NMR $\left(151 \mathrm{MHz}, \mathrm{CDCl}_{3}\right) \delta 167.8,154.2,134.6,128.6,128.6,128.4$, 99.6, 76.0, 73.2, 72.9, 70.5, 66.5, 66.1, 64.5, 62.7, 61.7, 52.0, 50.2, 14.0; HRMS (ESI+) m/z: $[\mathrm{M}+\mathrm{H}]^{+}$Calcd. for $\left[\mathrm{C}_{26} \mathrm{H}_{36} \mathrm{NO}_{12}\right.$ 554.2237; Found 554.2220; IR (thin film, $\left.\mathrm{cm}^{-1}\right)$ : 3236, 2954, 2848, 2360, 2334, 1749, 1541, 1267, 749; TLC $\left(\mathrm{MeOH}^{\mathrm{C}} \mathrm{CH}_{2} \mathrm{Cl} 2\right.$ $=5 / 95): R_{f}=0.14$.

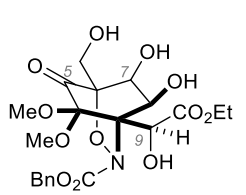

$( \pm)-13$

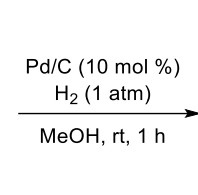

yield $86 \%$

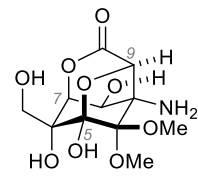

$( \pm)-24$

(士)-Ethyl 2-(1-amino-3,4,5,6-tetrahydroxy-4-(hydroxymethyl)-2,2-dimethoxycyclohexyl)-2-hydroxyacetate (24): A 1dram vial equipped with a magnetic stir bar was charged with tetrol 13 (50.0 mg, $0.103 \mathrm{mmol}, 1.0$ equiv), MeOH (1.0 mL), and palladium on charcoal ( $10 \% \mathrm{w} / \mathrm{w}, 11.0 \mathrm{mg}, 0.0103 \mathrm{mmol}, 0.10$ equiv). The vessel was sealed with a rubber septum and the mixture was sparged with a balloon of hydrogen gas for $10 \mathrm{~min}$. After stirring for an additional 50 min, the reaction mixture was filtered through a pad of Celite ${ }^{\circledR}$ with EtOAc and the filtrate was concentrated in vacuo to provide the title compound as a white foam $(27.1 \mathrm{mg}, 0.0886 \mathrm{mmol}, 86 \%)$. No further purification was required.

Analytical data: ${ }^{1} \mathrm{H}$ NMR $(400 \mathrm{MHz}, \mathrm{MeOD}) \delta 4.36(\mathrm{~d}, J=3.5 \mathrm{~Hz}, 1 \mathrm{H}), 4.33(\mathrm{dd}, J=3.5,1.2 \mathrm{~Hz}, 1 \mathrm{H}), 4.03(\mathrm{~d}, J=11.7 \mathrm{~Hz}$, $1 \mathrm{H}), 3.93(\mathrm{~d}, J=1.2 \mathrm{~Hz}, 1 \mathrm{H}), 3.75(\mathrm{~d}, J=11.7 \mathrm{~Hz}, 1 \mathrm{H}), 3.66(\mathrm{~s}, 3 \mathrm{H}), 3.62(\mathrm{~s}, 3 \mathrm{H}) . ;{ }^{13} \mathrm{C}\left\{{ }^{1} \mathrm{H}\right\} \mathrm{NMR}(151 \mathrm{MHz}, \mathrm{MeOD}) \delta 172.1$, 105.5, 101.7, 82.4, 81.0, 75.4, 66.6, 65.9, 64.2, 52.8, 51.9; HRMS (ESI+) m/z: [M + H] ${ }^{+}$Calcd. for $\mathrm{C}_{11} \mathrm{H}_{18} \mathrm{NO}_{9} 308.0981$; Found: 308.0970 . 


\section{References}

(1) Hansen, T. V.; Skattebøl, L. Ortho-Formylation of Phenols; Preparation of 3-Bromosalicylaldehyde. Org. Synth. 2005, 82, 64. https://doi.org/10.15227/orgsyn.082.0064.

(2) Vitaku, E.; Njardarson, J. T. A Mild Meta-Selective C-H Alkylation of Catechol Mono-Ethers. Eur. J. Org. Chem. 2016, 2016 (22), 3679-3683. https://doi.org/10.1002/ejoc.201600760.

(3) Bigi, F.; Casiraghi, G.; Casnati, G.; Sartori, G.; Fava, G. G.; Belicchi, M. F. Asymmetric Electrophilic Substitution on Phenols. 1. Enantioselective Ortho-Hydroxyalkylation Mediated by Chiral Alkoxyaluminum Chlorides. J. Org. Chem. 1985, 50 (25), 5018-5022. https://doi.org/10.1021/jo00225a003.

(4) Butler, J. R.; Wang, C.; Bian, J.; Ready, J. M. Enantioselective Total Synthesis of (-)-Kibdelone C. J. Am. Chem. Soc. 2011, 133 (26), 9956-9959. https://doi.org/10.1021/ja204040k.

(5) Wright, B. J. D.; Hartung, J.; Peng, F.; Van De Water, R.; Liu, H.; Tan, Q. H.; Chou, T. C.; Danishefsky, S. J. Synthesis of Pluraflavin A "Aglycone." J. Am. Chem. Soc. 2008, 130 (49), 16786-16790. https://doi.org/10.1021/ja805936v.

(6) Radosevich, A. T.; Musich, C.; Toste, F. D. Vanadium-Catalyzed Asymmetric Oxidation of a-Hydroxy Esters Using Molecular Oxygen as Stoichiometric Oxidant. J. Am. Chem. Soc. 2005, 127 (4), 1090-1091. https://doi.org/10.1021/ja0433424.

(7) Nicewicz, D. A.; Satterfield, A. D.; Schmitt, D. C.; Johnson, J. S. Self-Consistent Synthesis of the Squalene Synthase Inhibitor Zaragozic Acid C via Controlled Oligomerization. J. Am. Chem. Soc. 2008, 130 (51), 17281-17283. https://doi.org/10.1021/ja808347q.

(8) Allen, M. A.; Ivanovich, R. A.; Polat, D. E.; Beauchemin, A. M. Synthesis of N-Oxyureas by Substitution and CopeType Hydroamination Reactions Using O-lsocyanate Precursors. Org. Lett. 2017, 19 (24), 6574-6577. https://doi.org/10.1021/acs.orglett.7b03288. 


\section{Crude ${ }^{1} \mathrm{H}$ NMR Spectra}

JGR20-TTX1-991_crd.1.fid

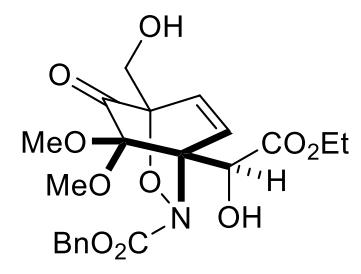

( \pm -12
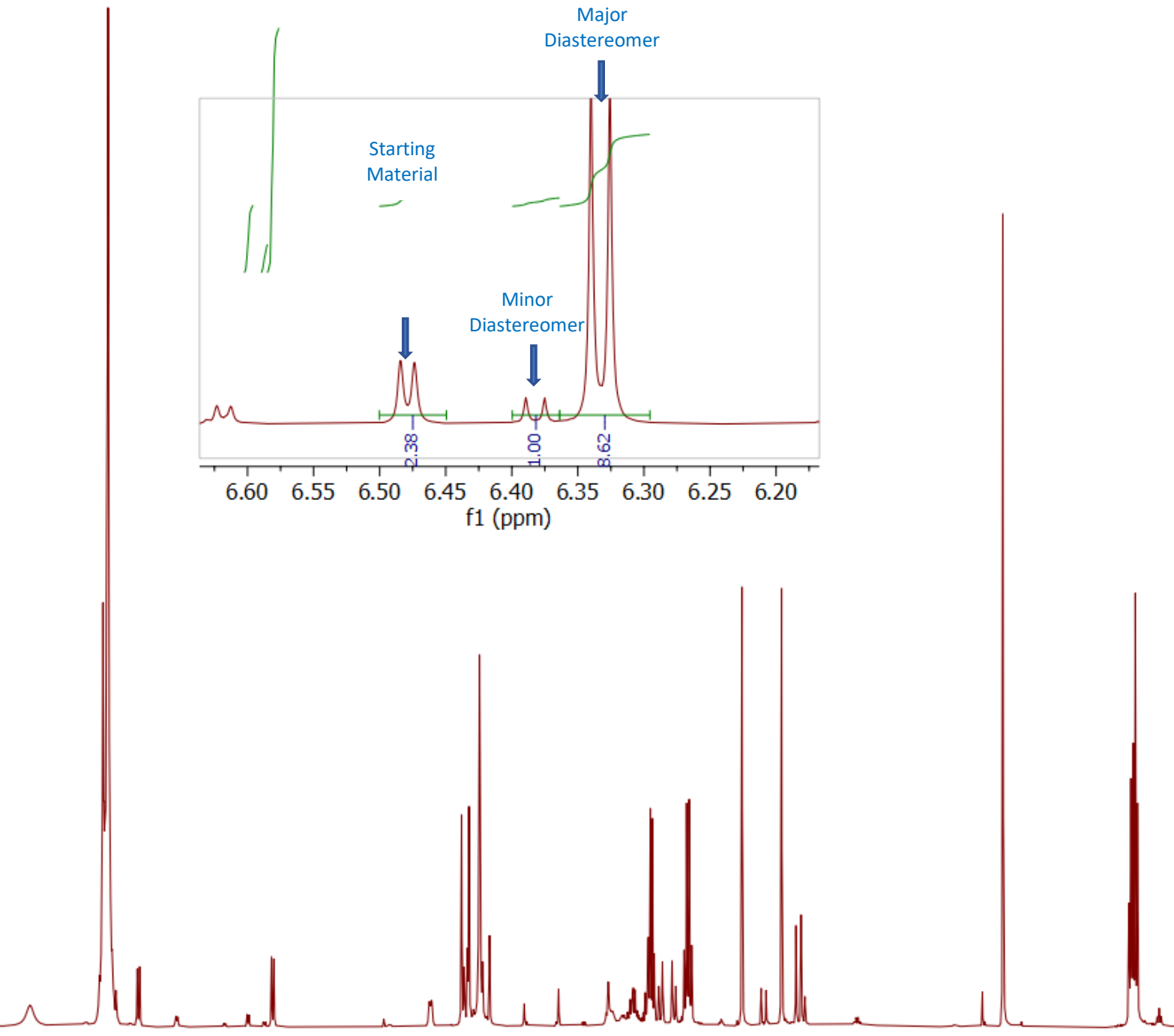

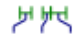

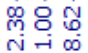

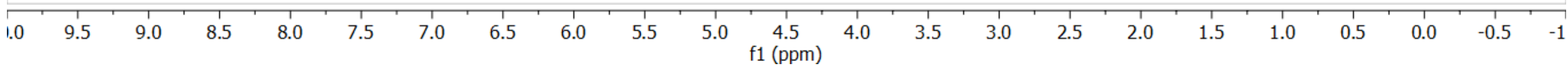




\section{Compound 14}

JGR20-TTX1-842_crd.1.fid

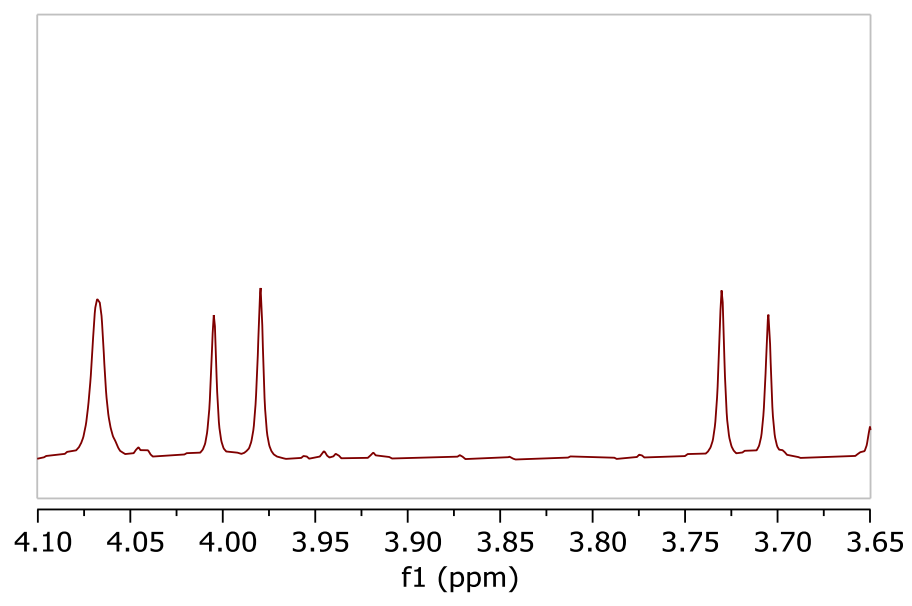

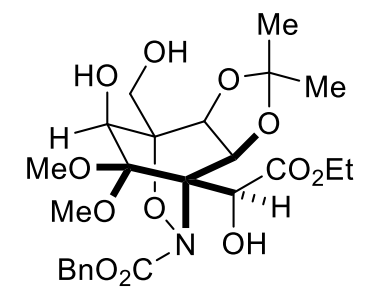

(土)-14

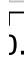

). 9.5

9.0

8.5

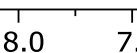

$7.5 \quad 7.0$

6.5

6.0

5.5

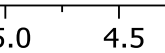
f1 (ppm) 


\section{Compound $\mathbf{S 6}$}

JGR20-TTX1-988_crd.1.fid
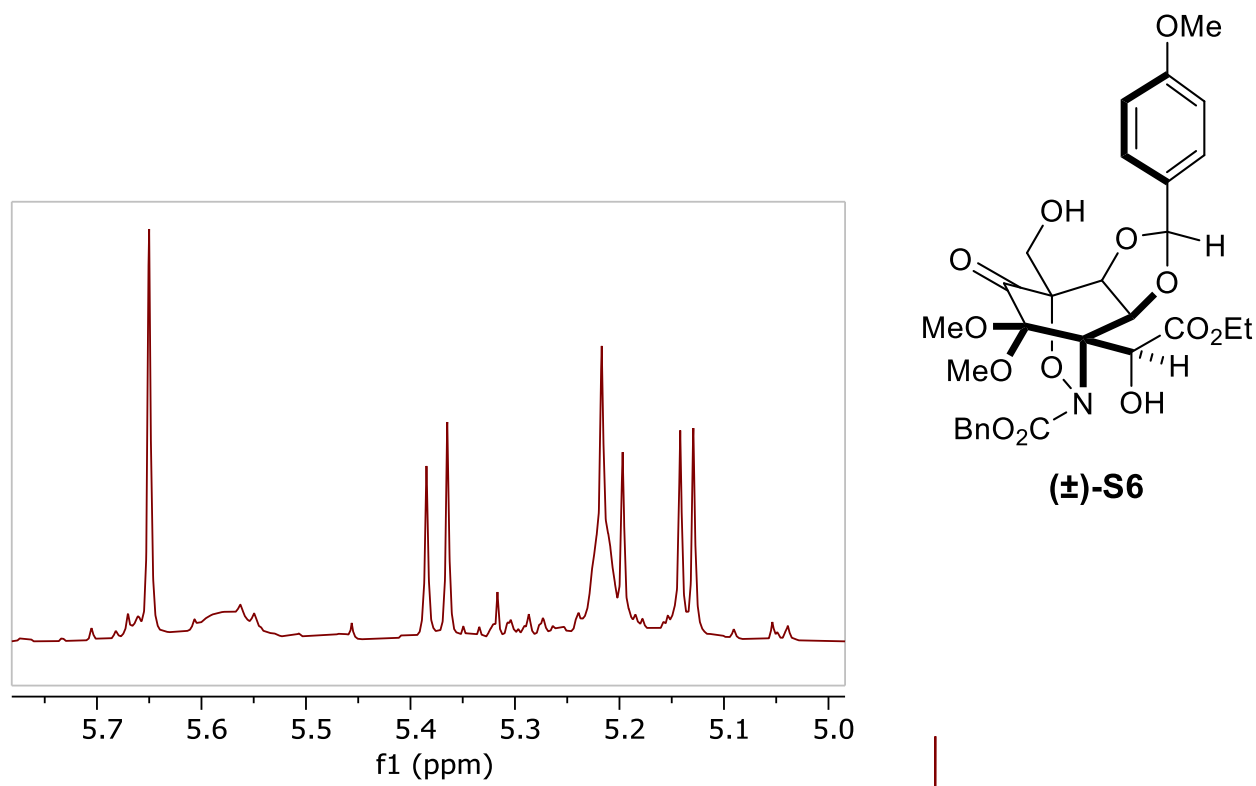

(士)-S6

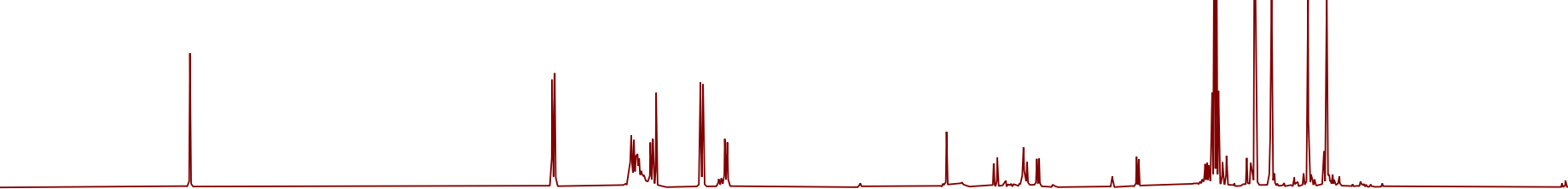

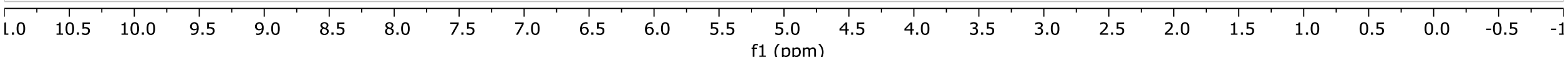

$\mathrm{S} 14$ 


\section{Compound 20}

JGR20-TTX1-1013_crd.1.fid
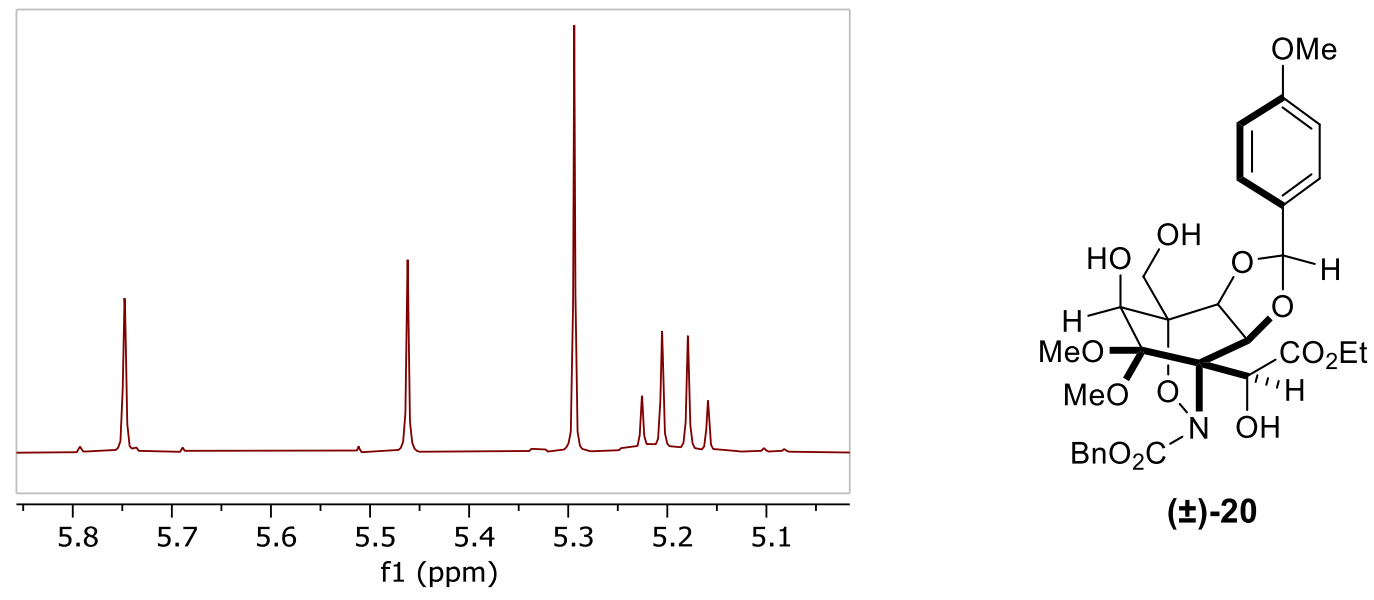

(士)-20

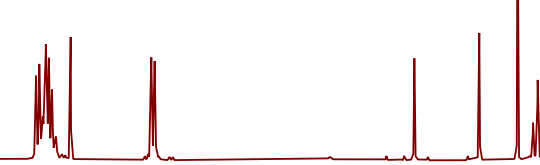

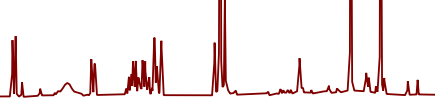
M. M.
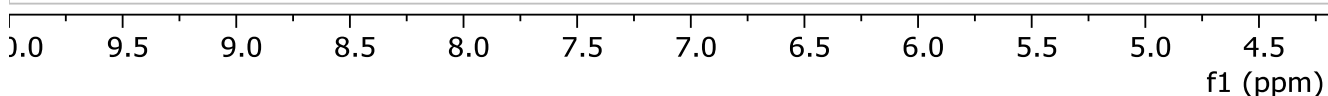

\begin{tabular}{|c|c|c|c|c|c|c|c|c|c|c|}
\hline & & & & & & & & & & \\
\hline 4.0 & 3.5 & 3.0 & 2.5 & 2.0 & 1.5 & 1.0 & 0.5 & 0.0 & -0.5 & -1 \\
\hline
\end{tabular}


${ }^{1} \mathrm{H},{ }^{13} \mathrm{C}$, and 2D NMR Spectra for Purified Compounds

Compound 6 ( ${ }^{1} \mathrm{H}, 600 \mathrm{MHz}, \mathrm{CDCl}_{3}, 12$ to $-1 \mathrm{ppm}$ )

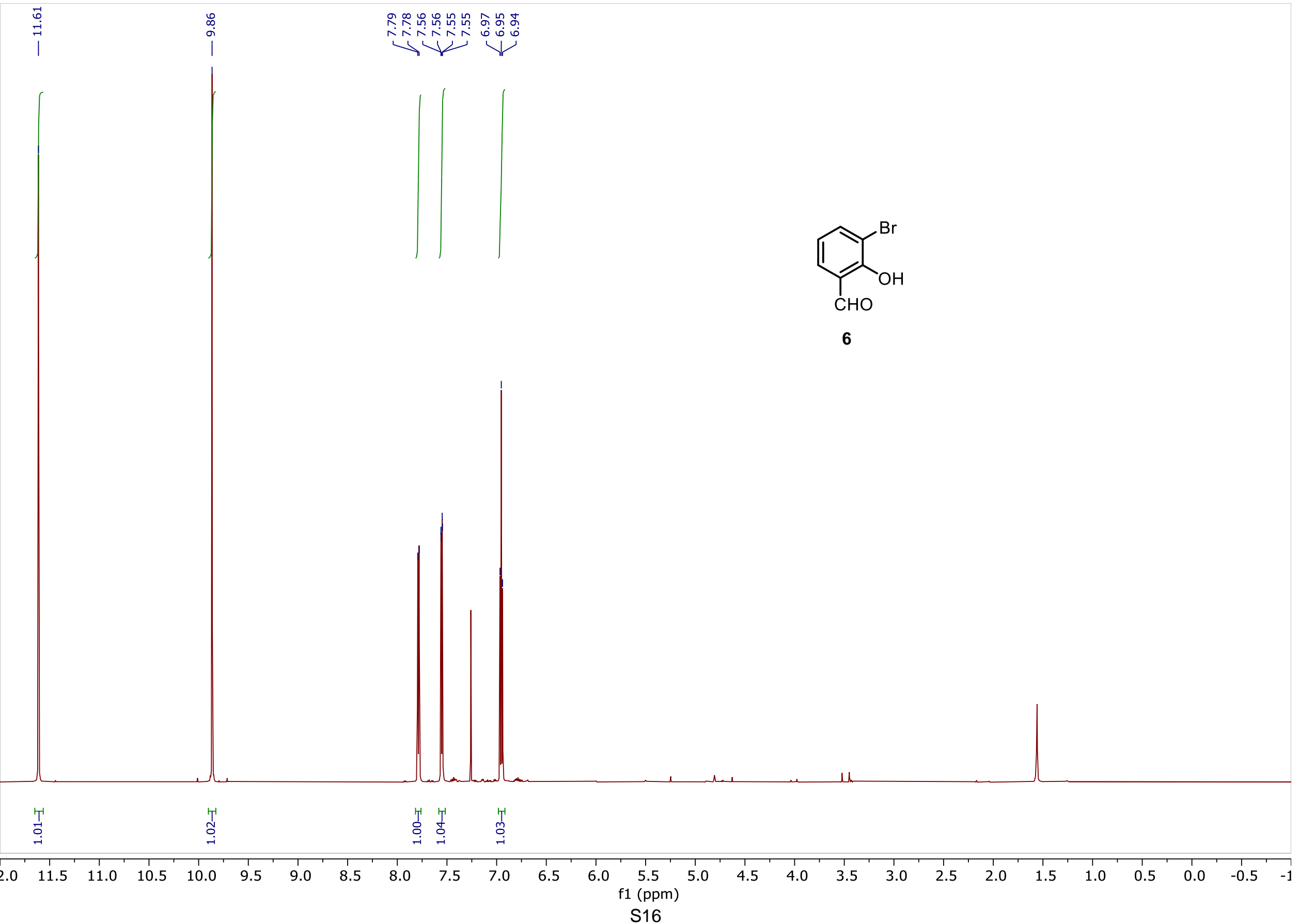


Compound S1 ( ${ }^{1} \mathrm{H}, 600 \mathrm{MHz}, \mathrm{CDCl}_{3}, 12$ to -1 ppm)

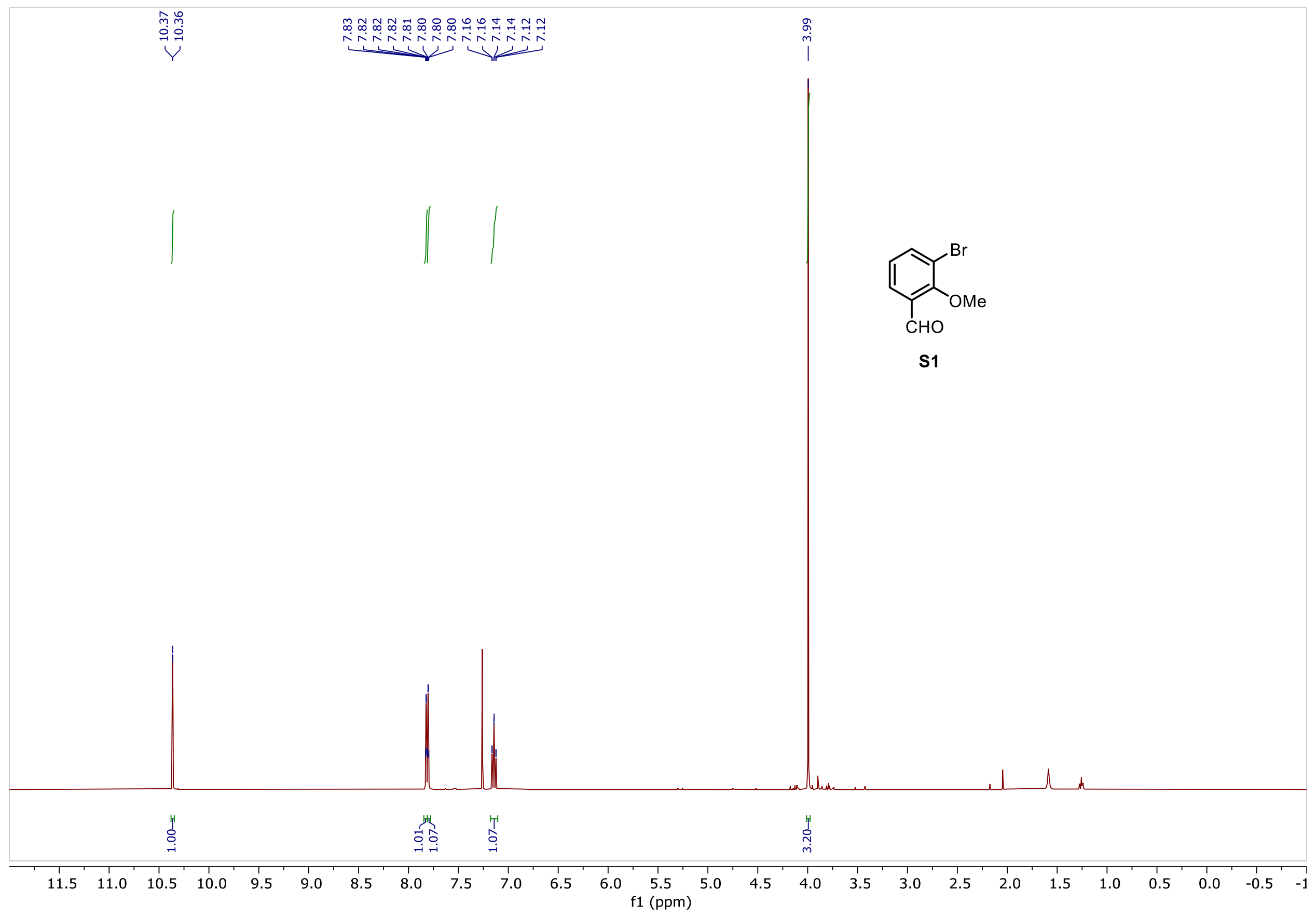


Compound 7 ( ${ }^{1} \mathrm{H}, 600 \mathrm{MHz}, \mathrm{CDCl}_{3}$,standard view)

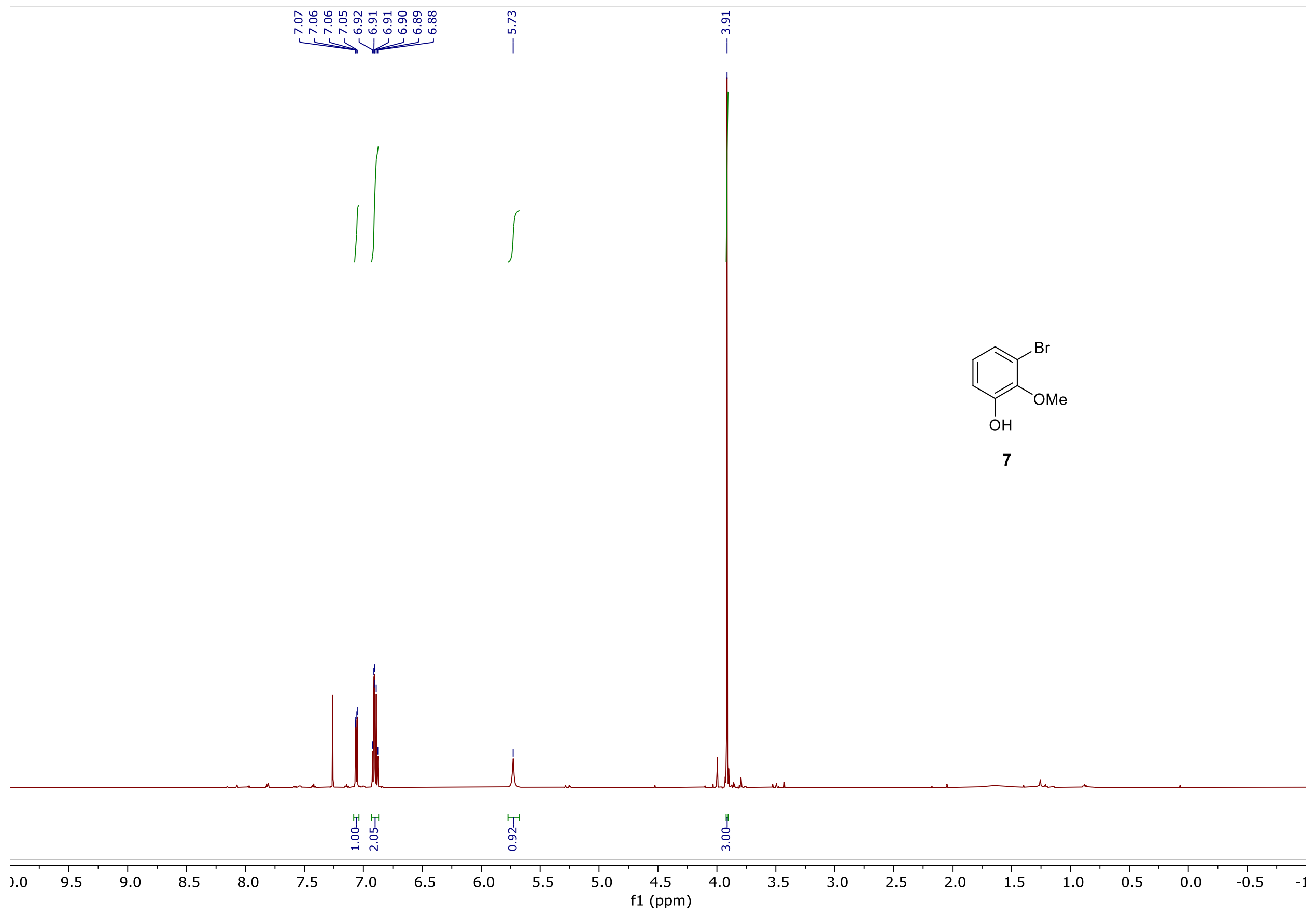


Compound $8\left({ }^{1} \mathrm{H}, 600 \mathrm{MHz}, \mathrm{CDCl}_{3}\right.$, standard view)

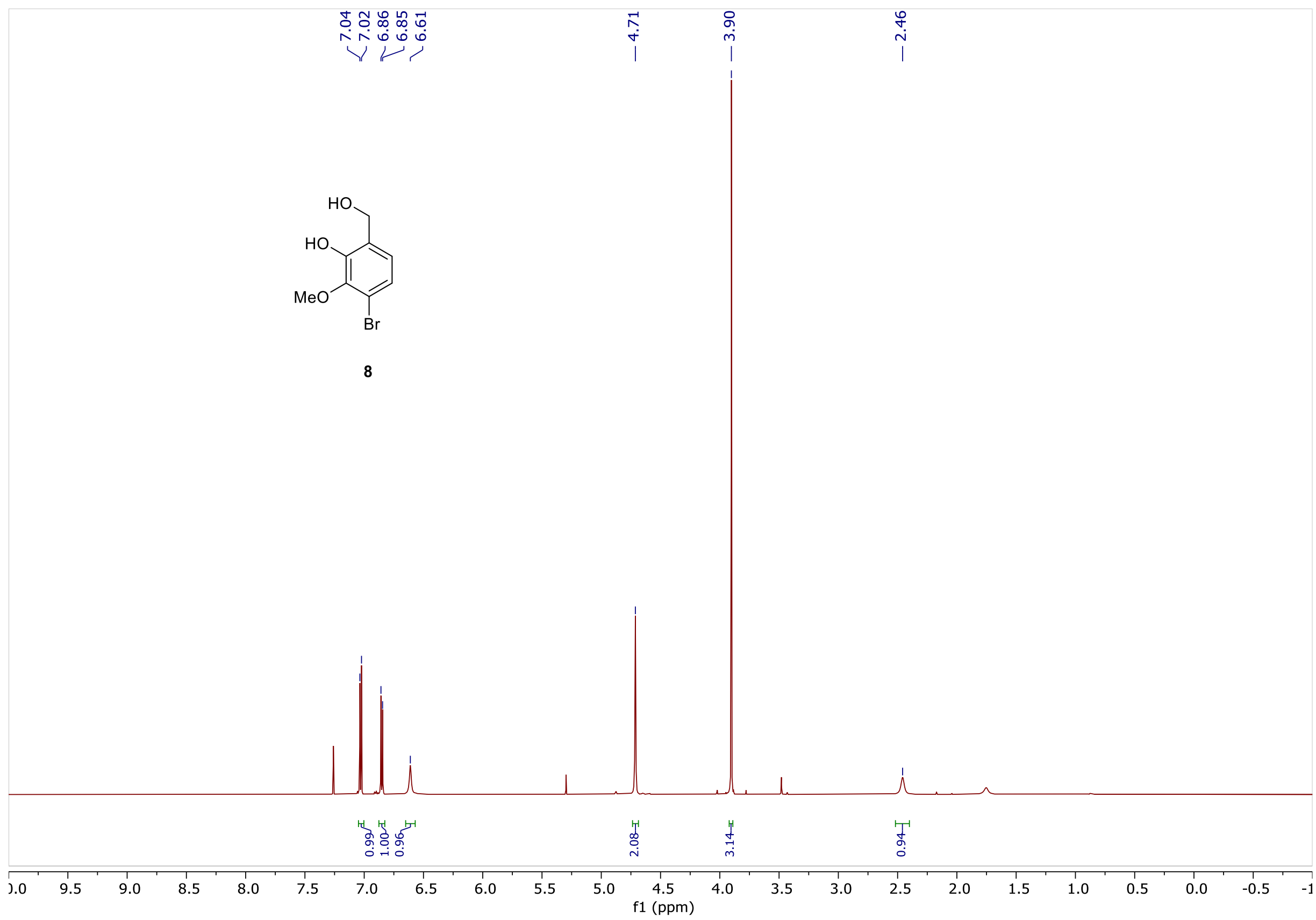


Compound $8\left({ }^{13} \mathrm{C}\left\{{ }^{1} \mathrm{H}\right\}, 151 \mathrm{MHz}, \mathrm{CDCl}_{3}\right.$, standard view)

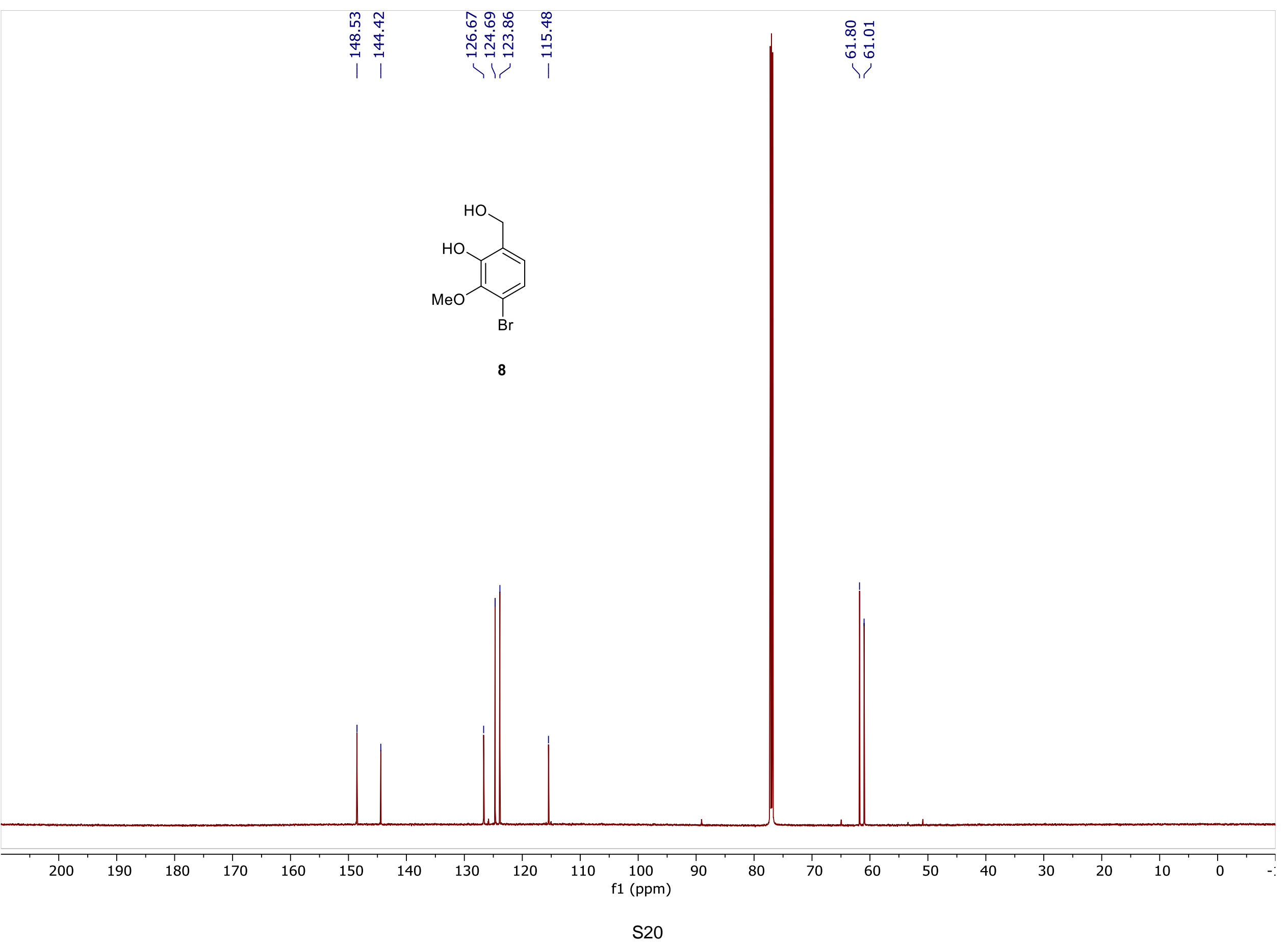




\section{Compound $9\left({ }^{1} \mathrm{H}, 600 \mathrm{MHz}, \mathrm{CDCl}_{3}\right.$, standard view)}

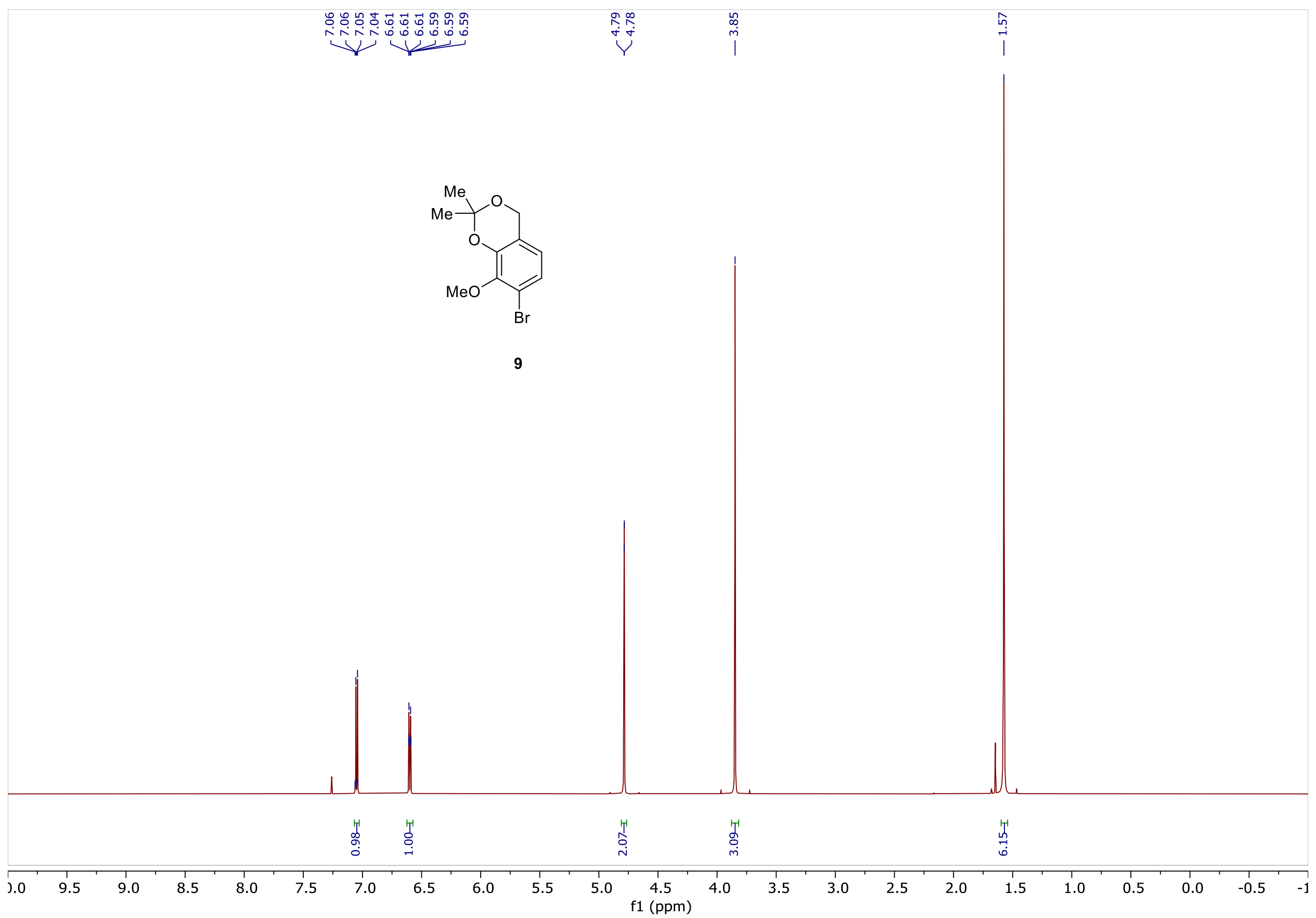


Compound $9\left({ }^{13} \mathrm{C}\left\{{ }^{1} \mathrm{H}\right\}, 151 \mathrm{MHz}, \mathrm{CDCl}_{3}\right.$, standard view)

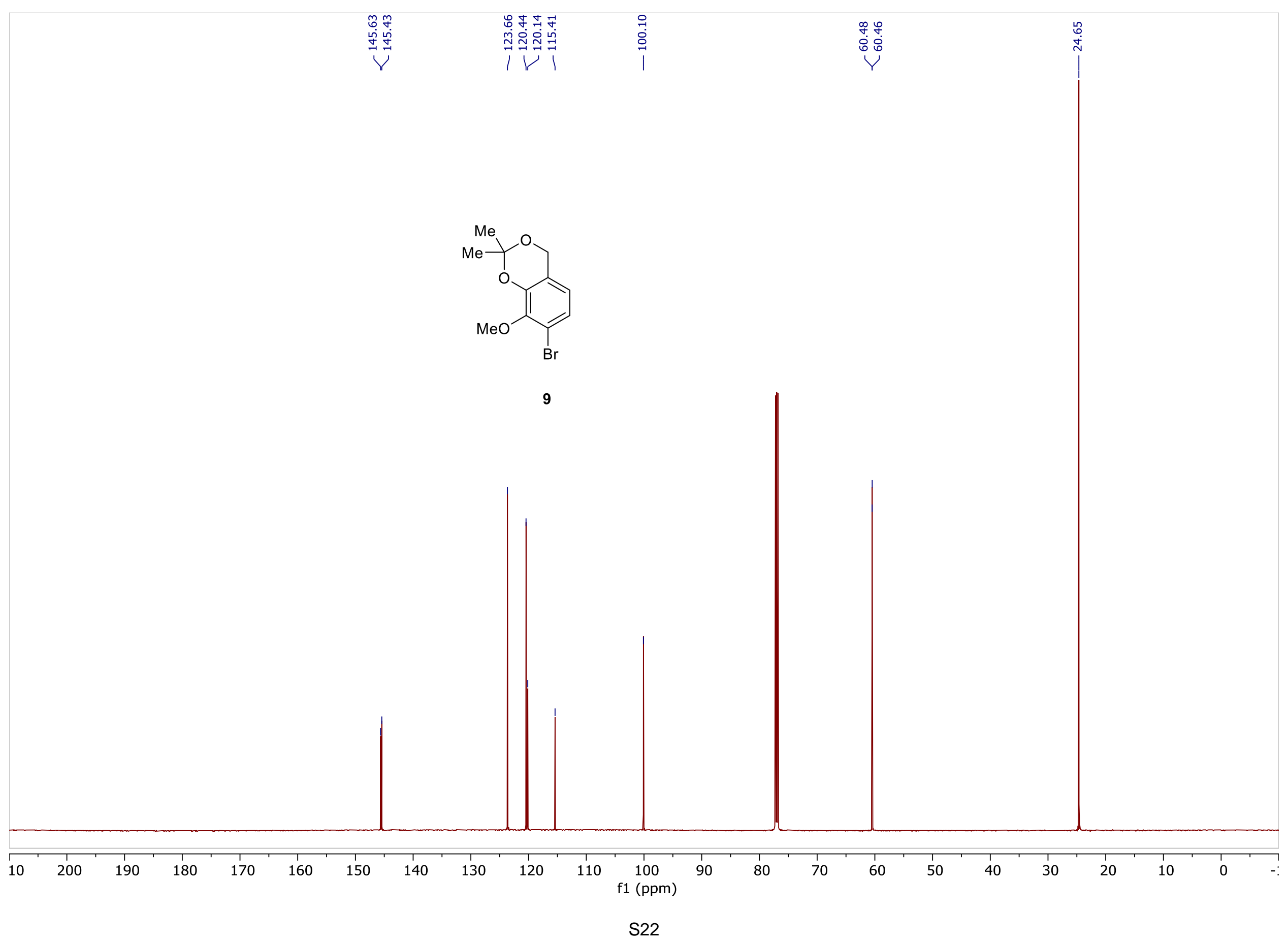


Compound $10\left({ }^{1} \mathrm{H}, 600 \mathrm{MHz}, \mathrm{CDCl}_{3}\right.$, standard view)

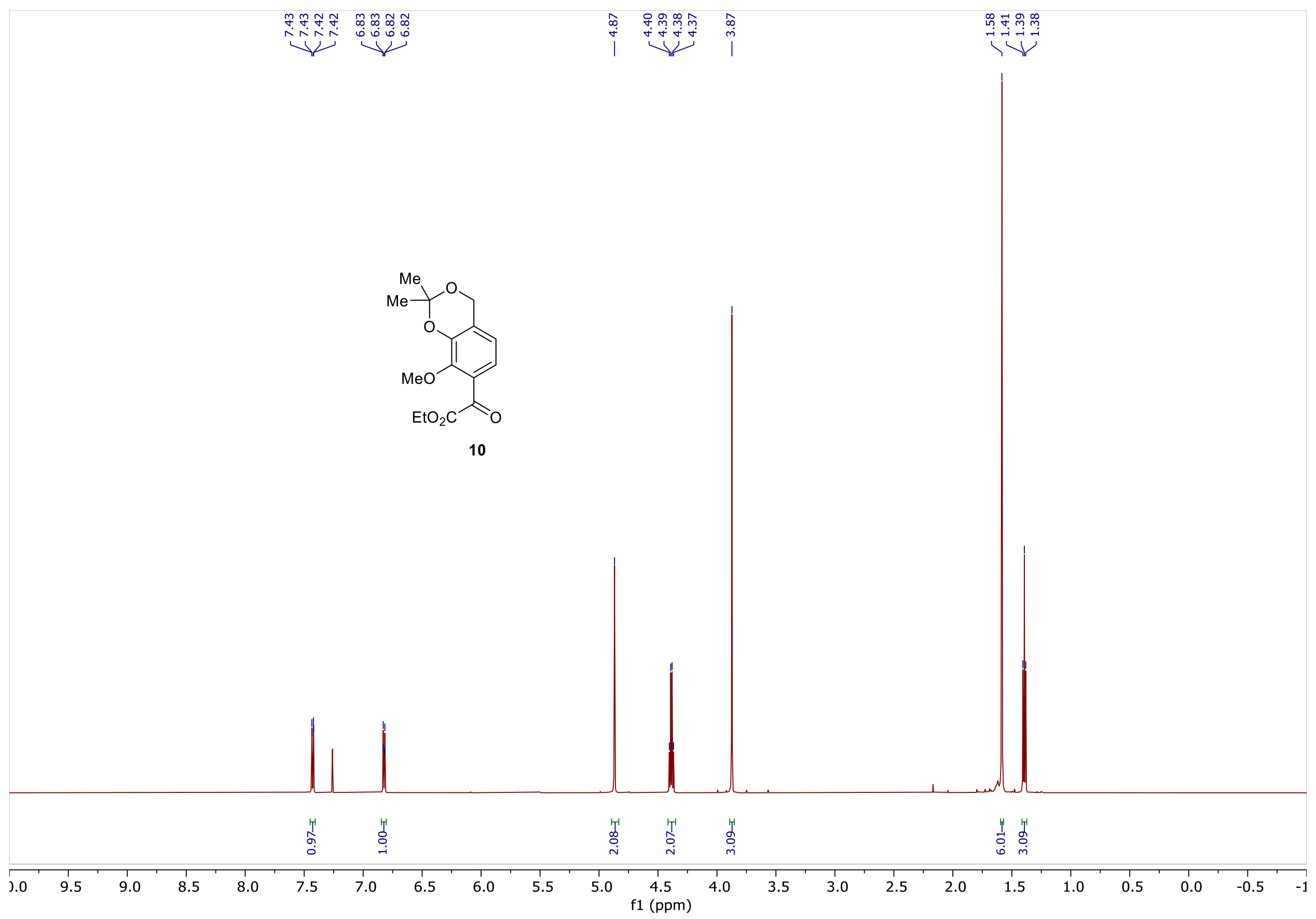


Compound $10\left({ }^{13} \mathrm{C}\left\{{ }^{1} \mathrm{H}\right\}, 151 \mathrm{MHz}, \mathrm{CDCl}_{3}\right.$, standard view)

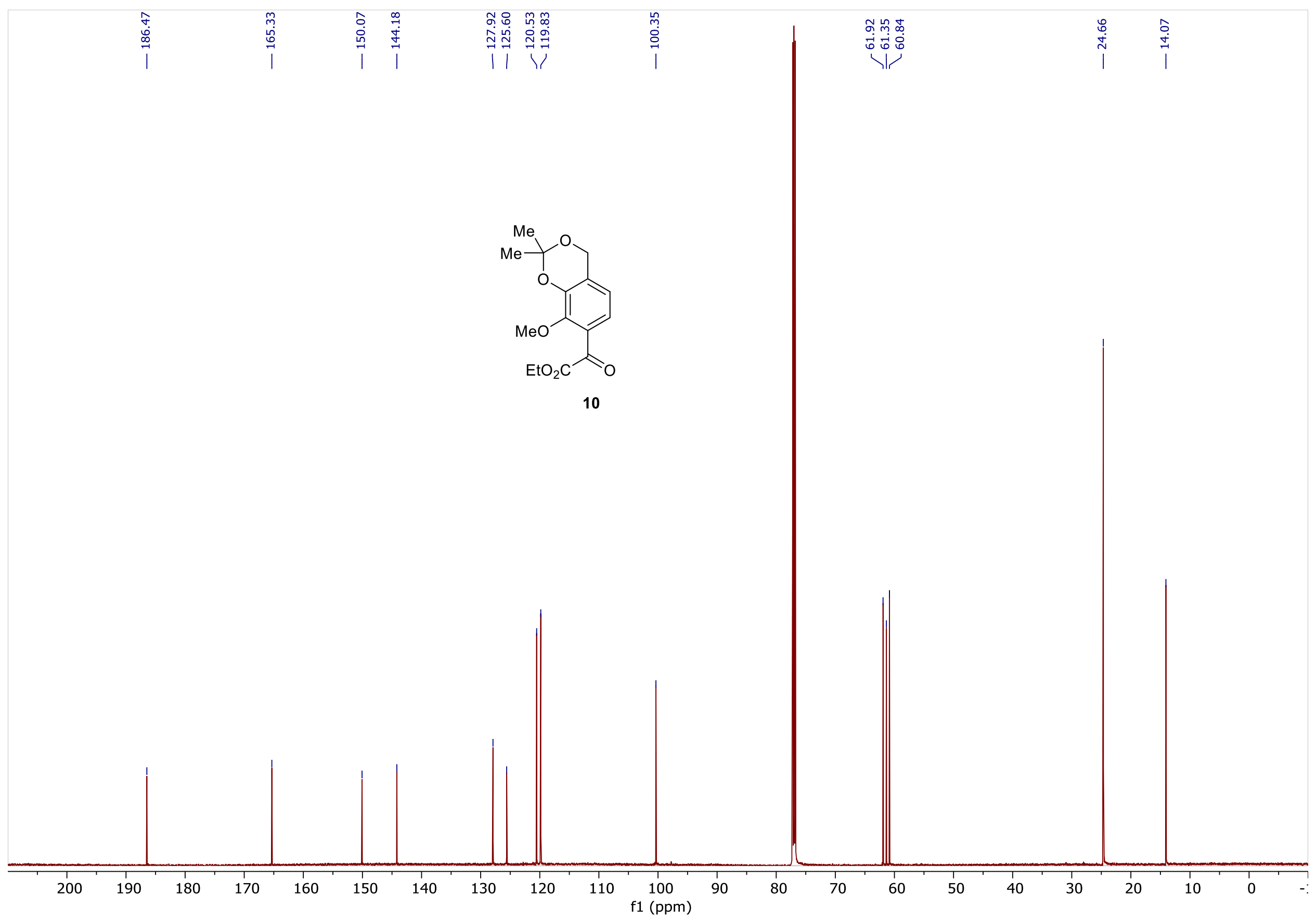


Compound $11\left({ }^{1} \mathrm{H}, 600 \mathrm{MHz}, \mathrm{CDCl}_{3}\right.$, standard view)

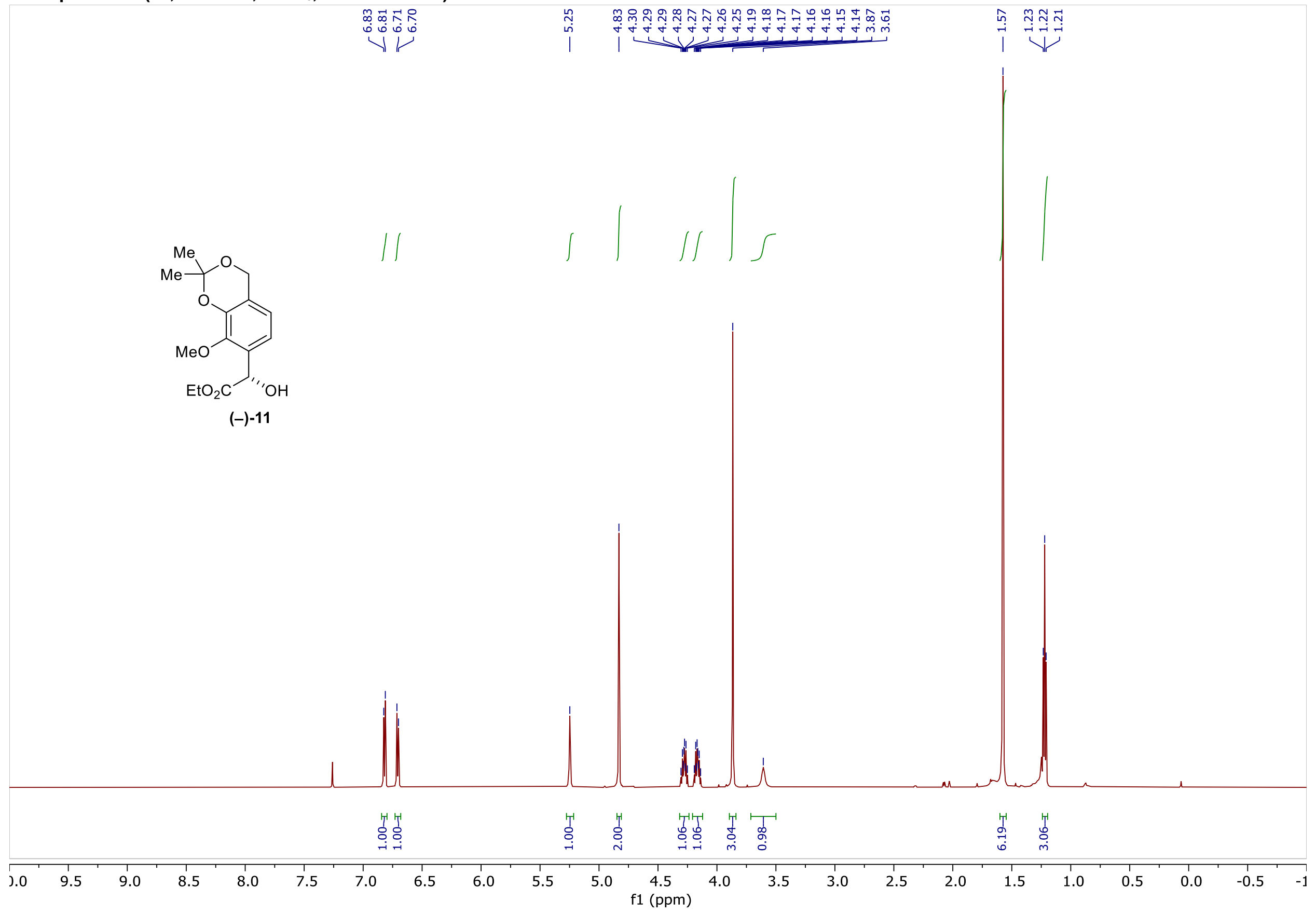


Compound $11\left({ }^{13} \mathrm{C}\left\{{ }^{1} \mathrm{H}\right\}, 151 \mathrm{MHz}, \mathrm{CDCl}_{3}\right.$, standard view)

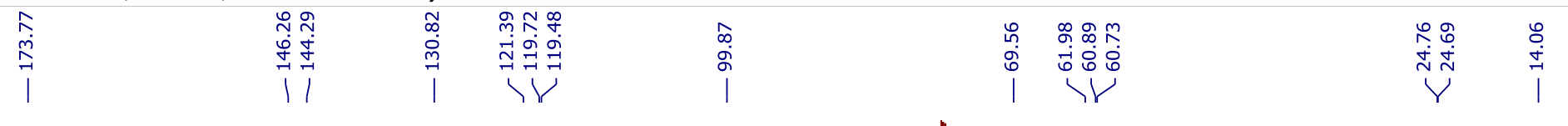

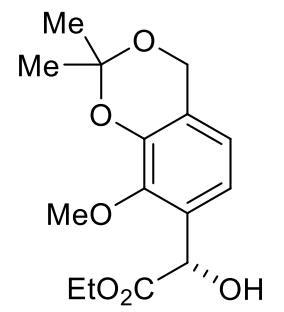

(-)-11
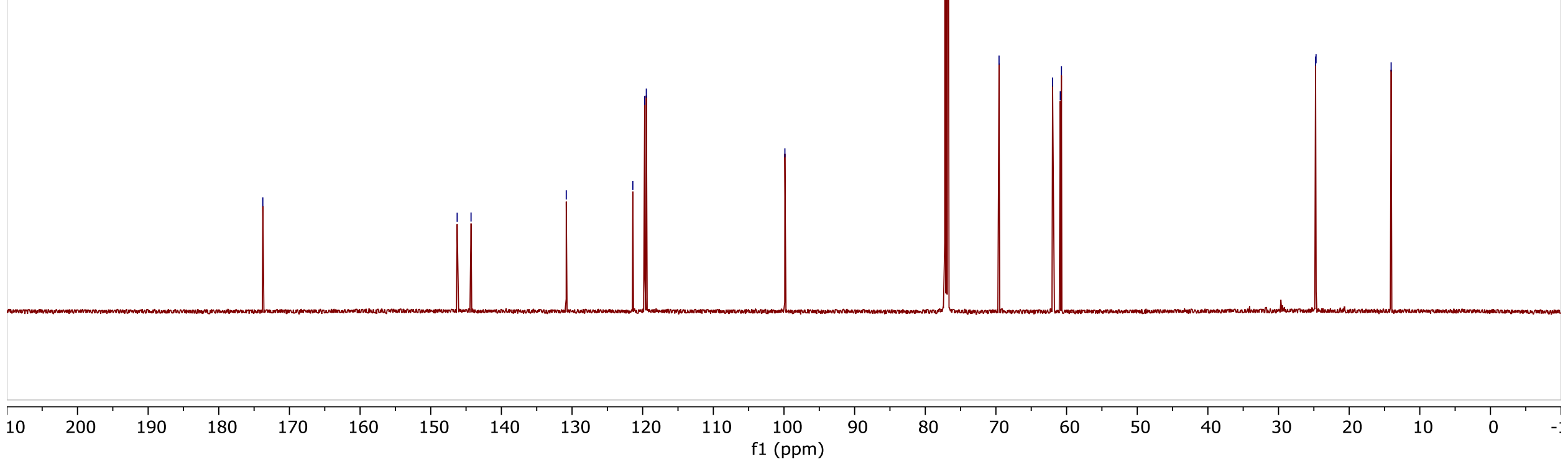
Compound ( \pm )-4 $\left({ }^{1} \mathrm{H}, 600 \mathrm{MHz}, \mathrm{CDCl}_{3}\right.$, standard view)

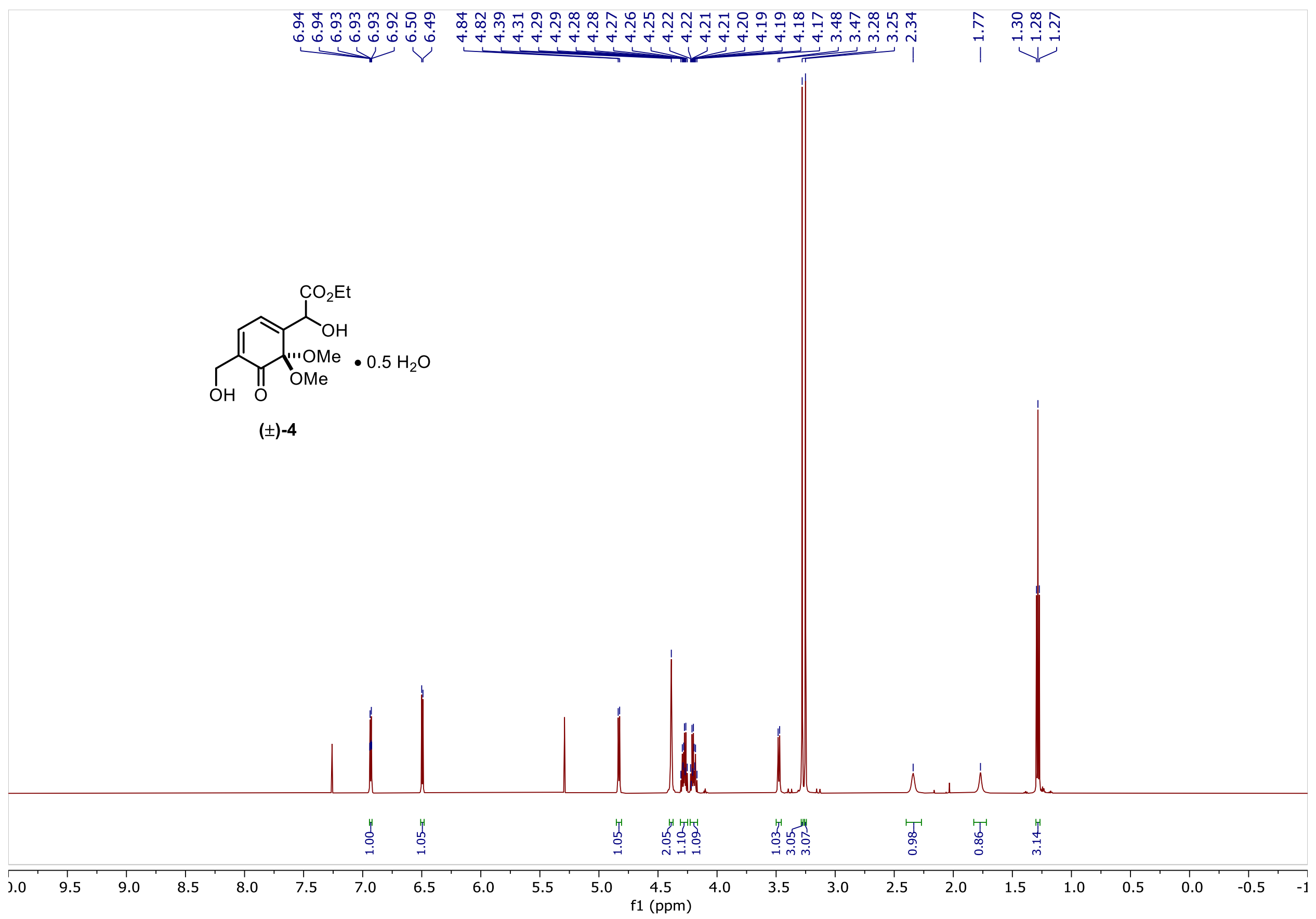


Compound $( \pm)-4\left({ }^{13} \mathrm{C}\left\{{ }^{1} \mathrm{H}\right\}, 151 \mathrm{MHz}, \mathrm{CDCl}_{3}\right.$, standard view)

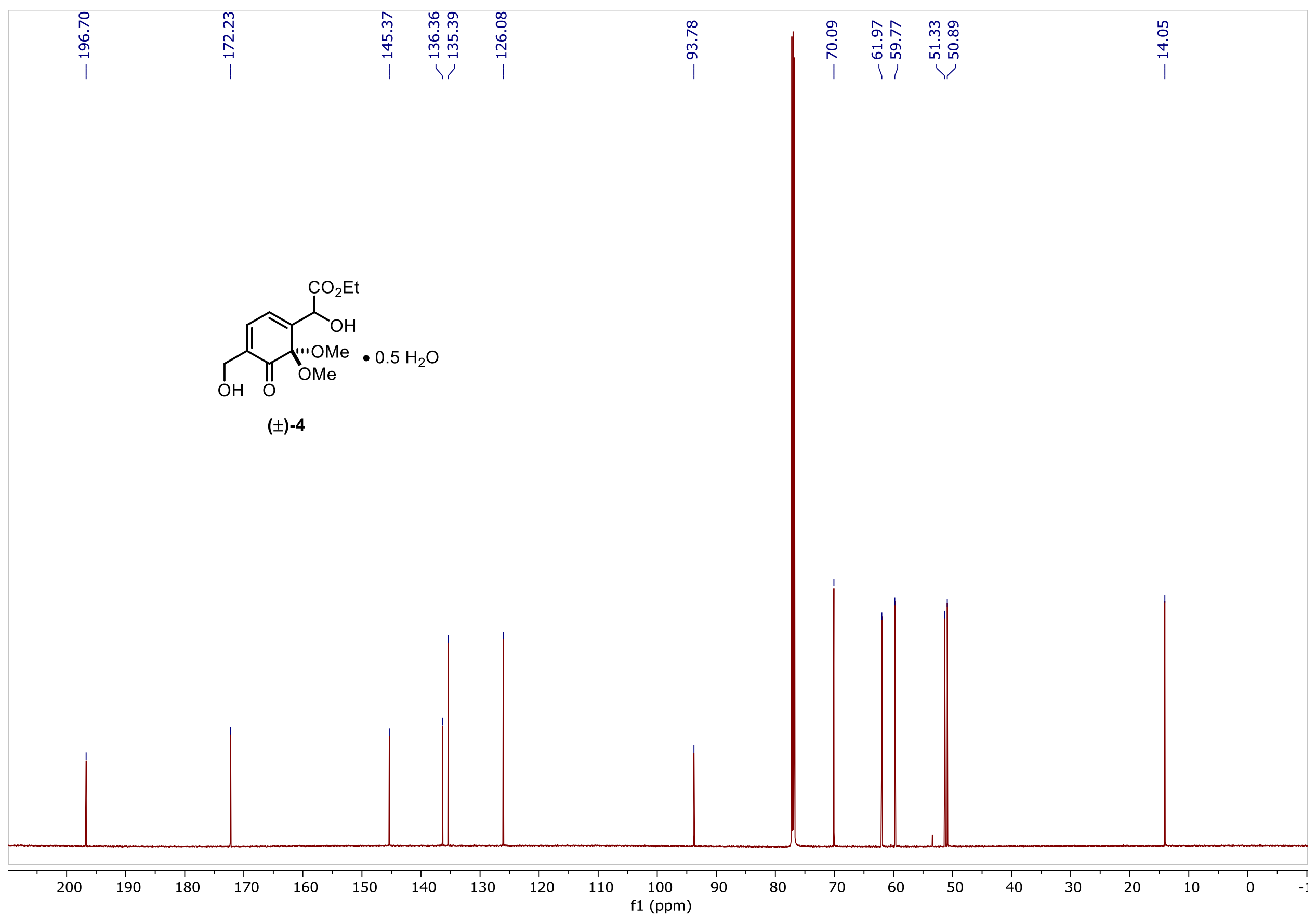


Benzyl hydroxycarbamate $\left({ }^{1} \mathrm{H}, 400 \mathrm{MHz}, \mathrm{CDCl}_{3}\right.$, standard view)

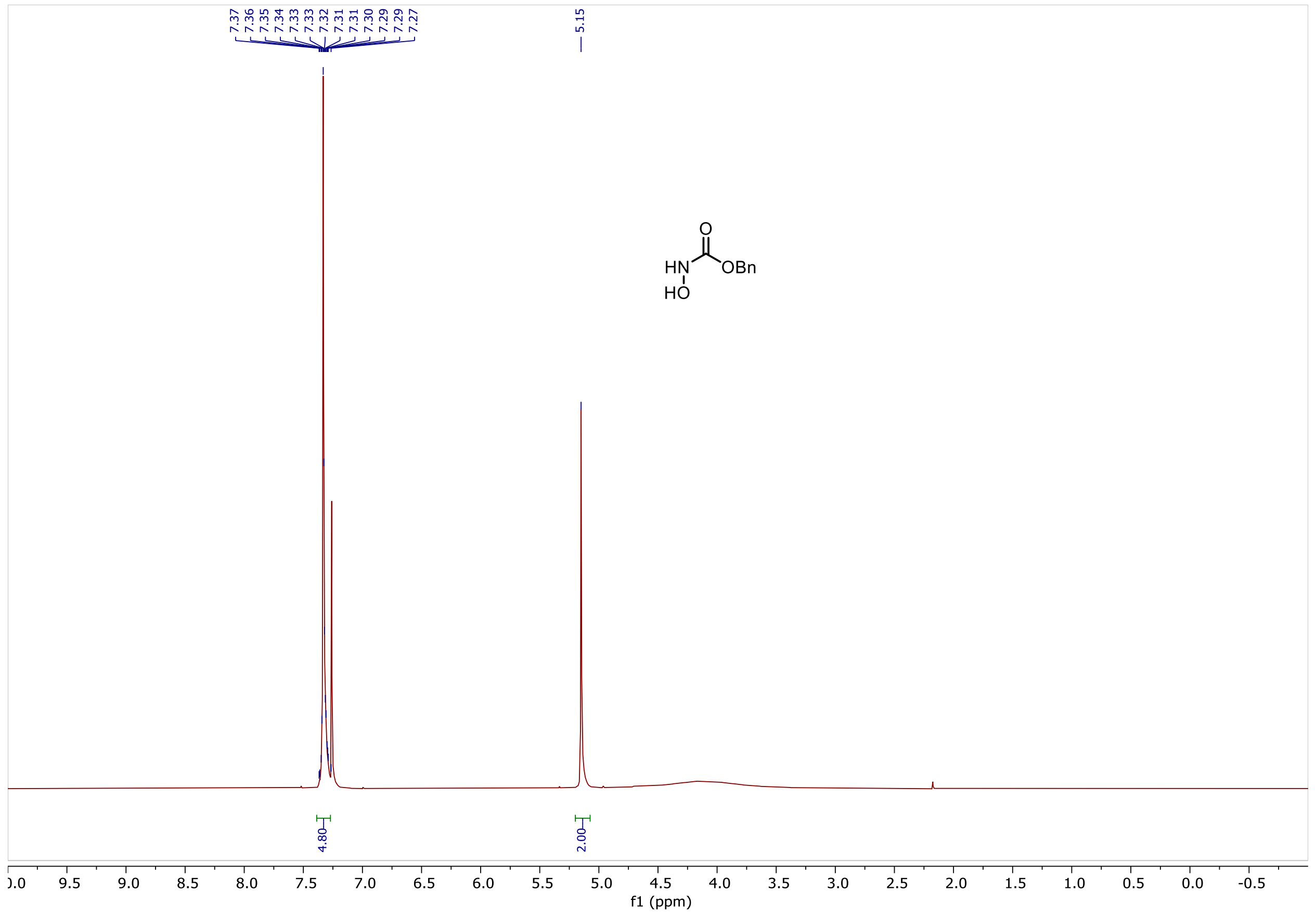




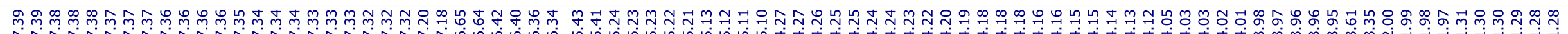

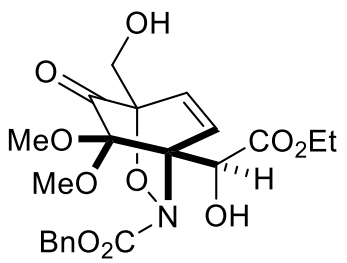

$( \pm)-12$

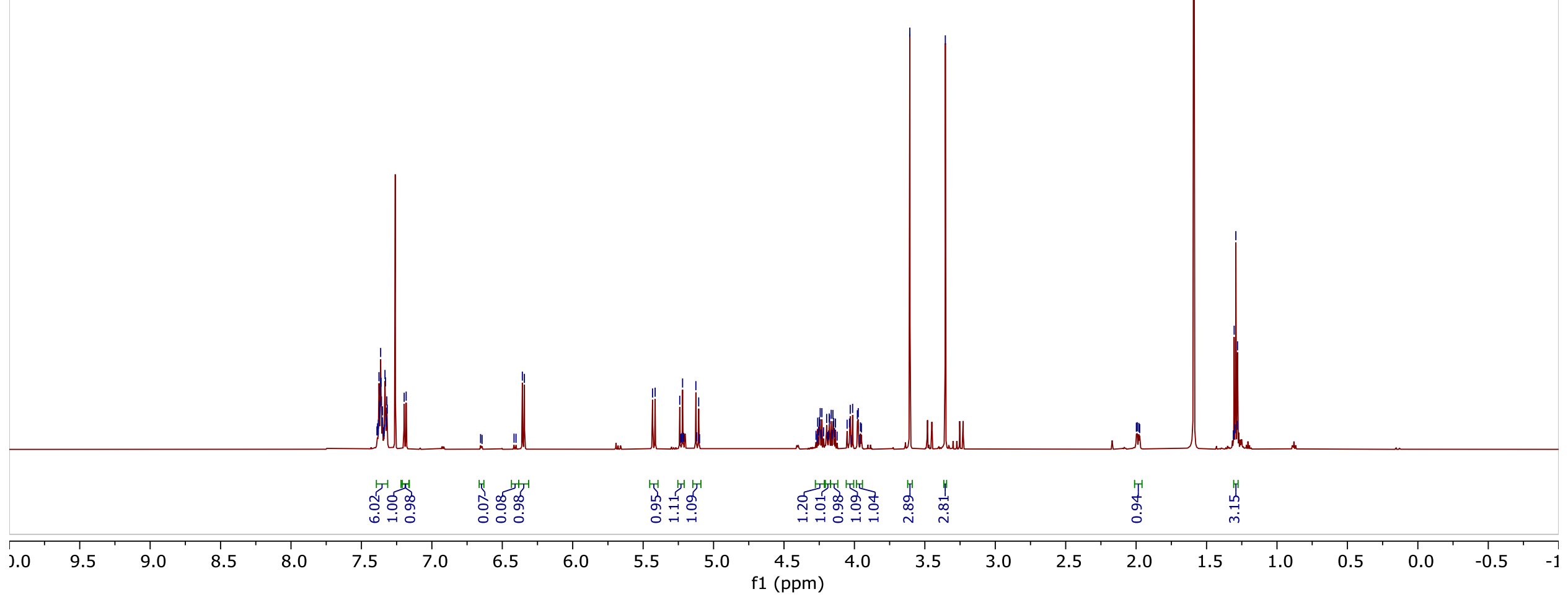


Compound ( \pm )-12 $\left({ }^{13} \mathrm{C}\left\{{ }^{1} \mathrm{H}\right\}, 151 \mathrm{MHz}, \mathrm{CDCl}_{3}\right.$, standard view)

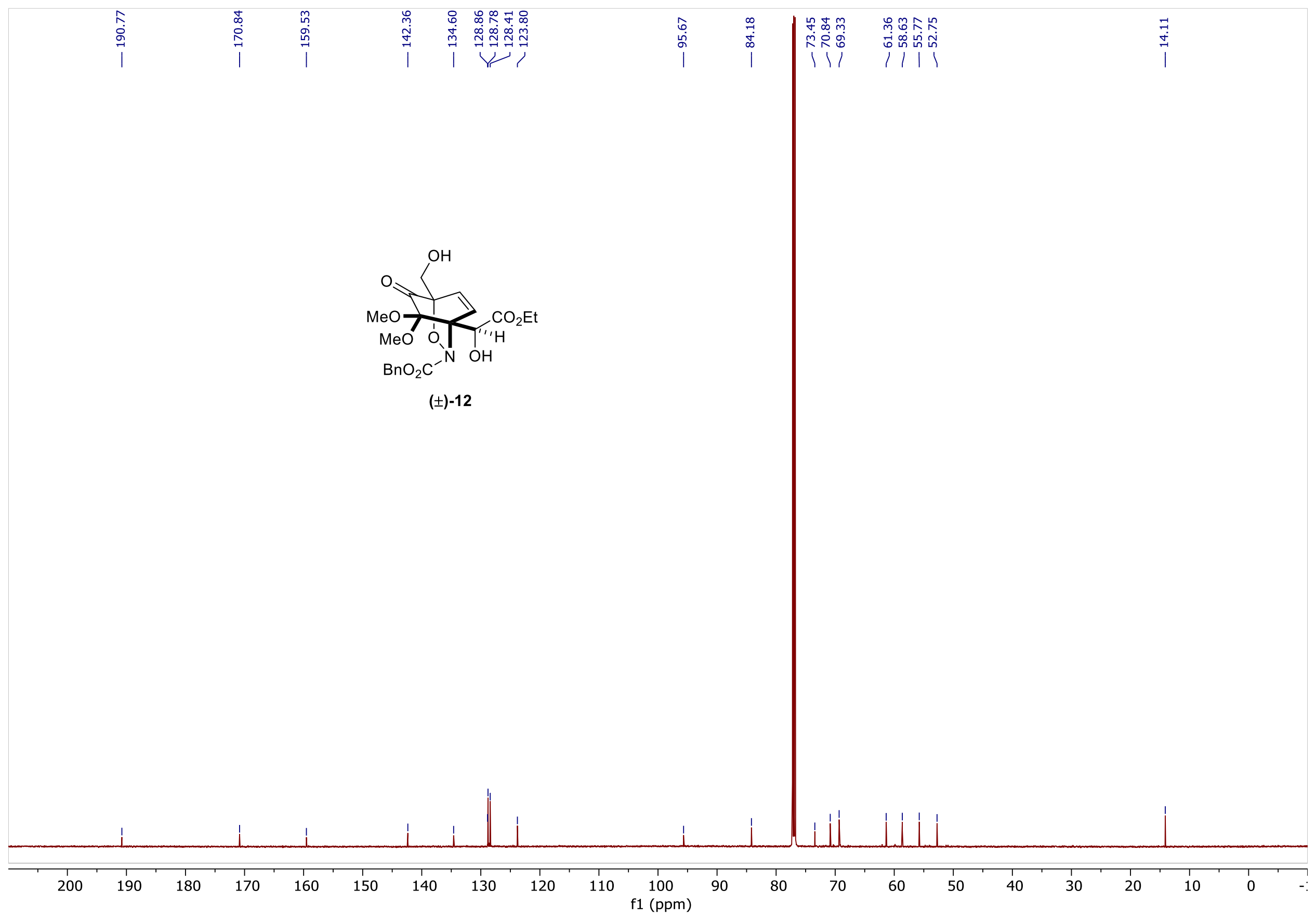




\section{Compound ( \pm )-13 ( ${ }^{1} \mathrm{H}, 600 \mathrm{MHz}, \mathrm{CDCl}_{3}$, standard view)}

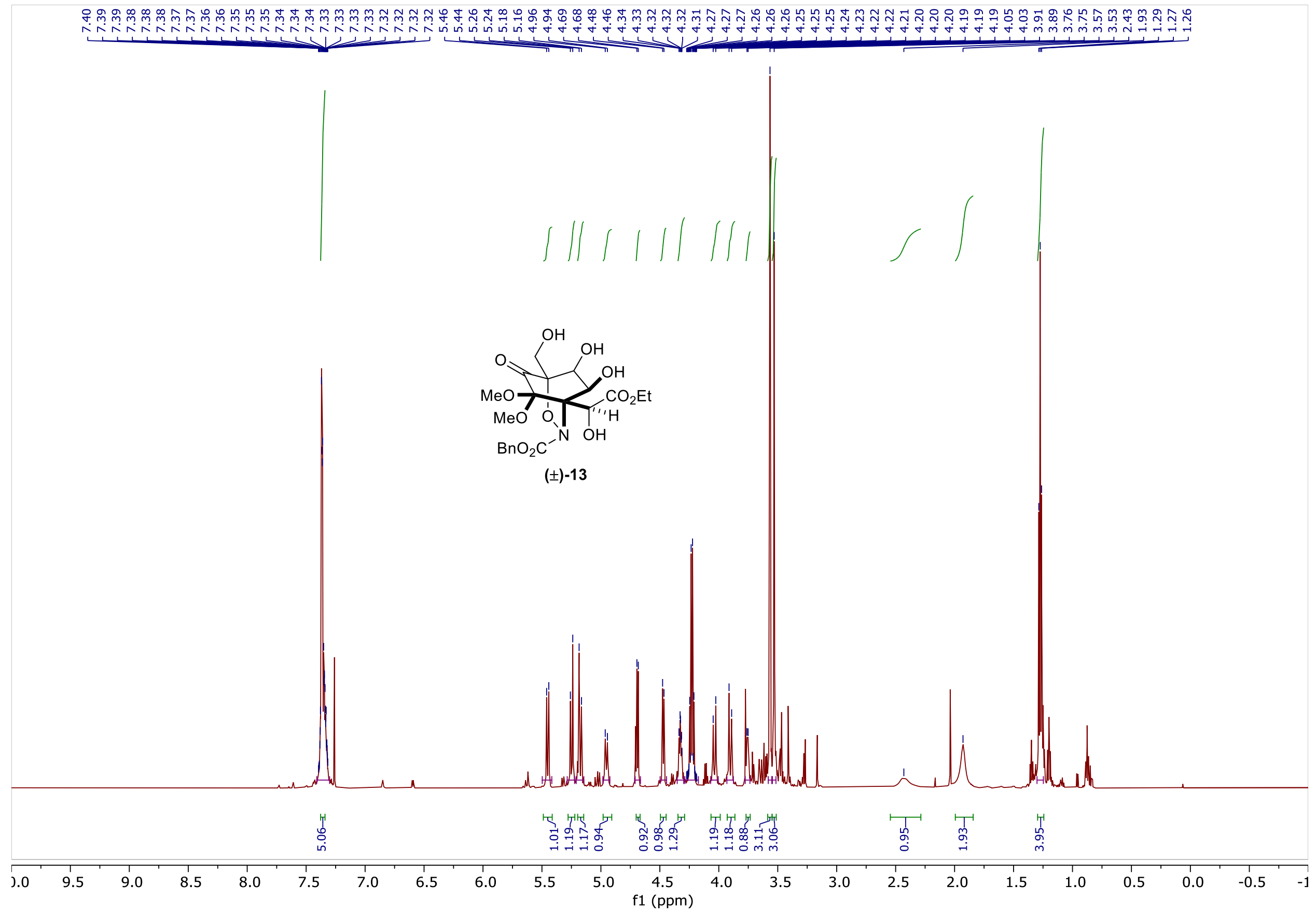


Compound ( \pm$)-13\left({ }^{13} \mathrm{C}\left\{{ }^{1} \mathrm{H}\right\}, 151 \mathrm{MHz}, \mathrm{CDCl}_{3}\right.$, standard view)

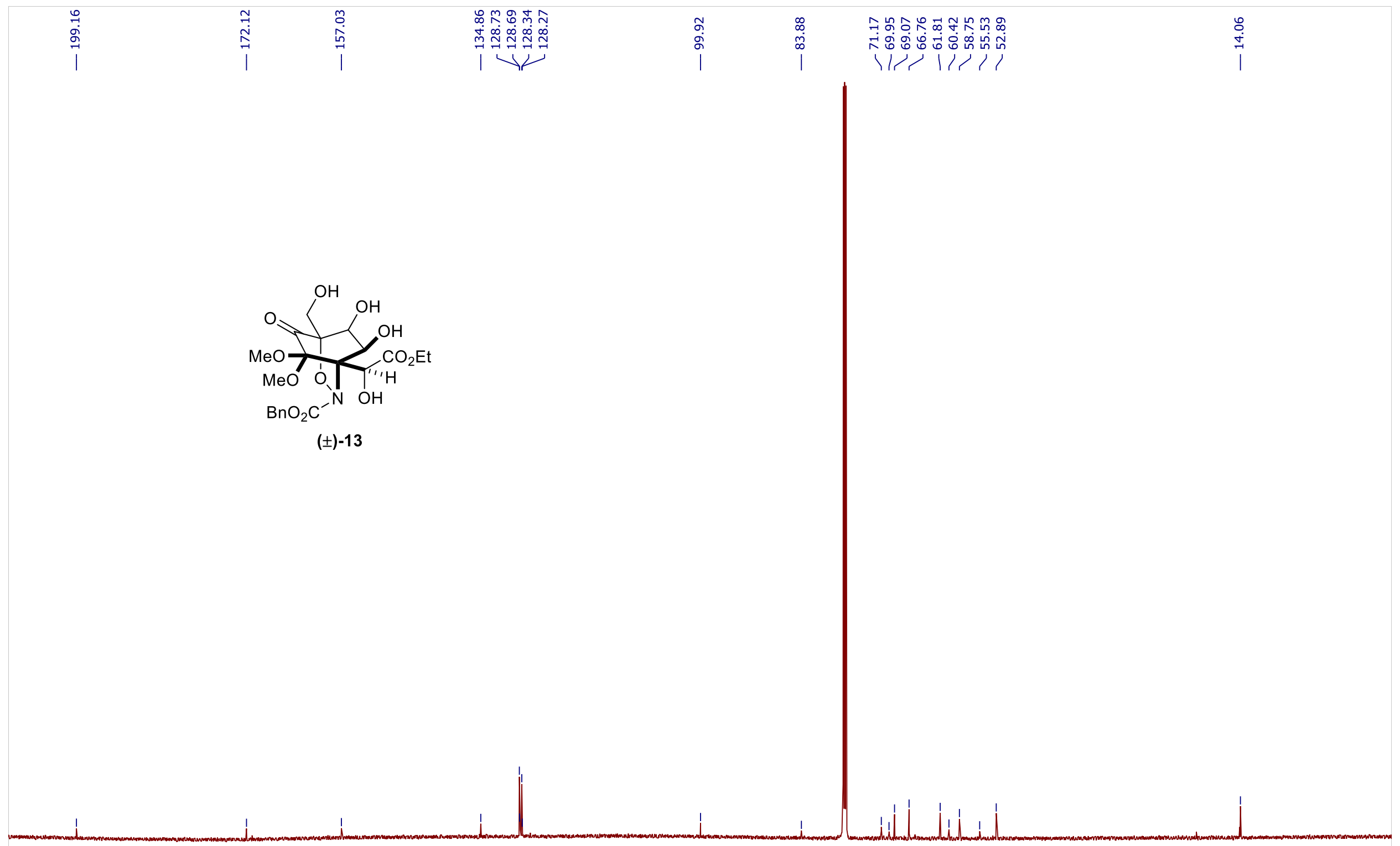

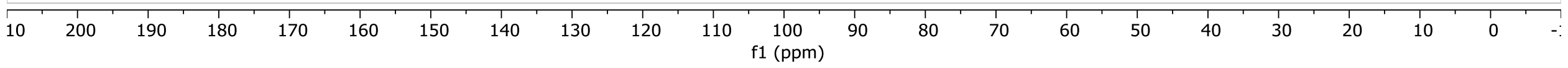




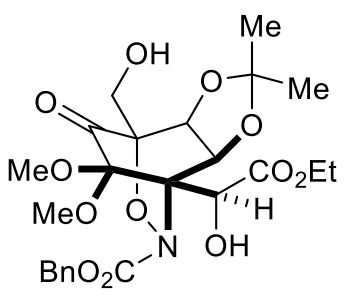

( \pm -S4

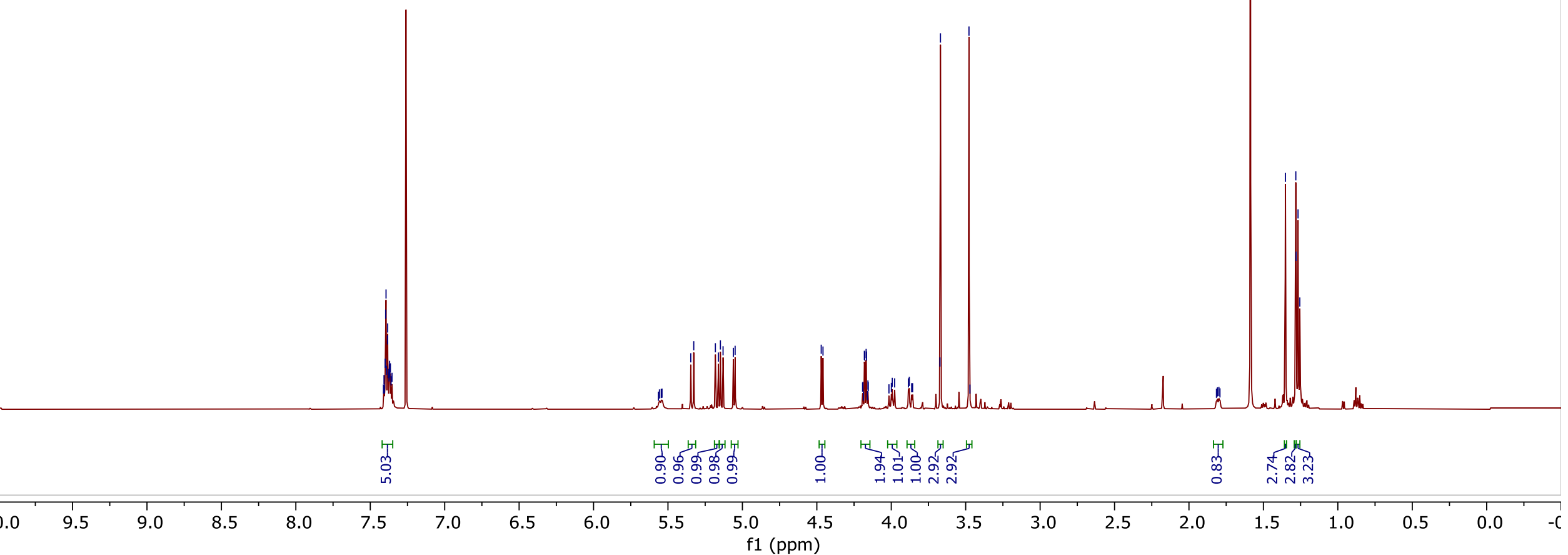


Compound ( \pm )-S4 $\left({ }^{13} \mathrm{C}\left\{{ }^{1} \mathrm{H}\right\}, 151 \mathrm{MHz}, \mathrm{CDCl}_{3}\right.$, standard view)

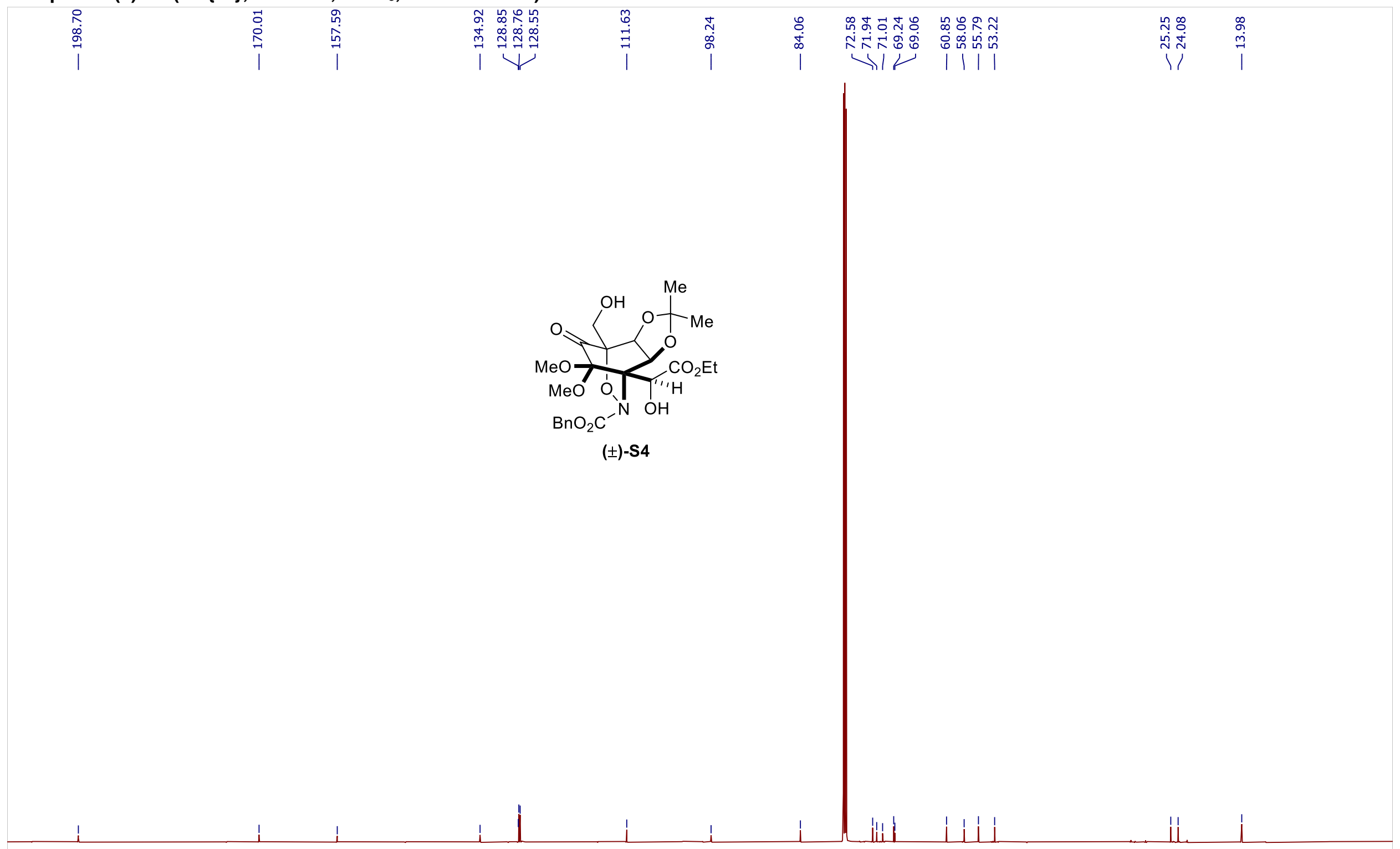

\begin{tabular}{|c|c|c|c|c|c|c|c|c|c|c|c|c|c|c|c|c|c|c|c|c|c|}
\hline 10 & 200 & 190 & 180 & 170 & 160 & 150 & 140 & 130 & 120 & 110 & $\begin{array}{c}100 \\
\mathrm{f} 1(\mathrm{ppm})\end{array}$ & 90 & 80 & 70 & 60 & 50 & 40 & 30 & 20 & 10 & 0 \\
\hline
\end{tabular}




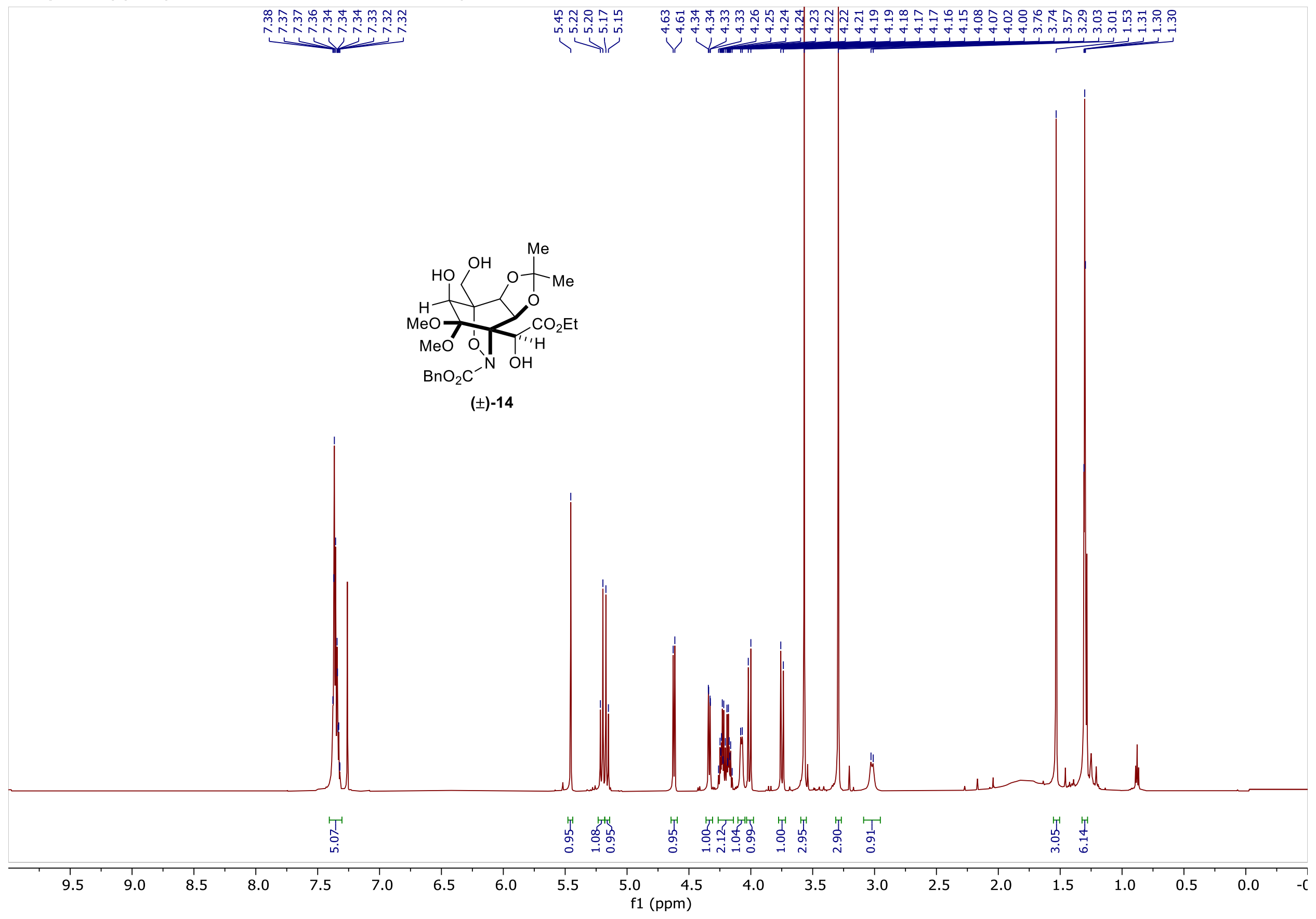


Compound ( \pm )-14 $\left({ }^{13} \mathrm{C}\left\{{ }^{1} \mathrm{H}\right\}, 151 \mathrm{MHz}, \mathrm{CDCl}_{3}\right.$, standard view)

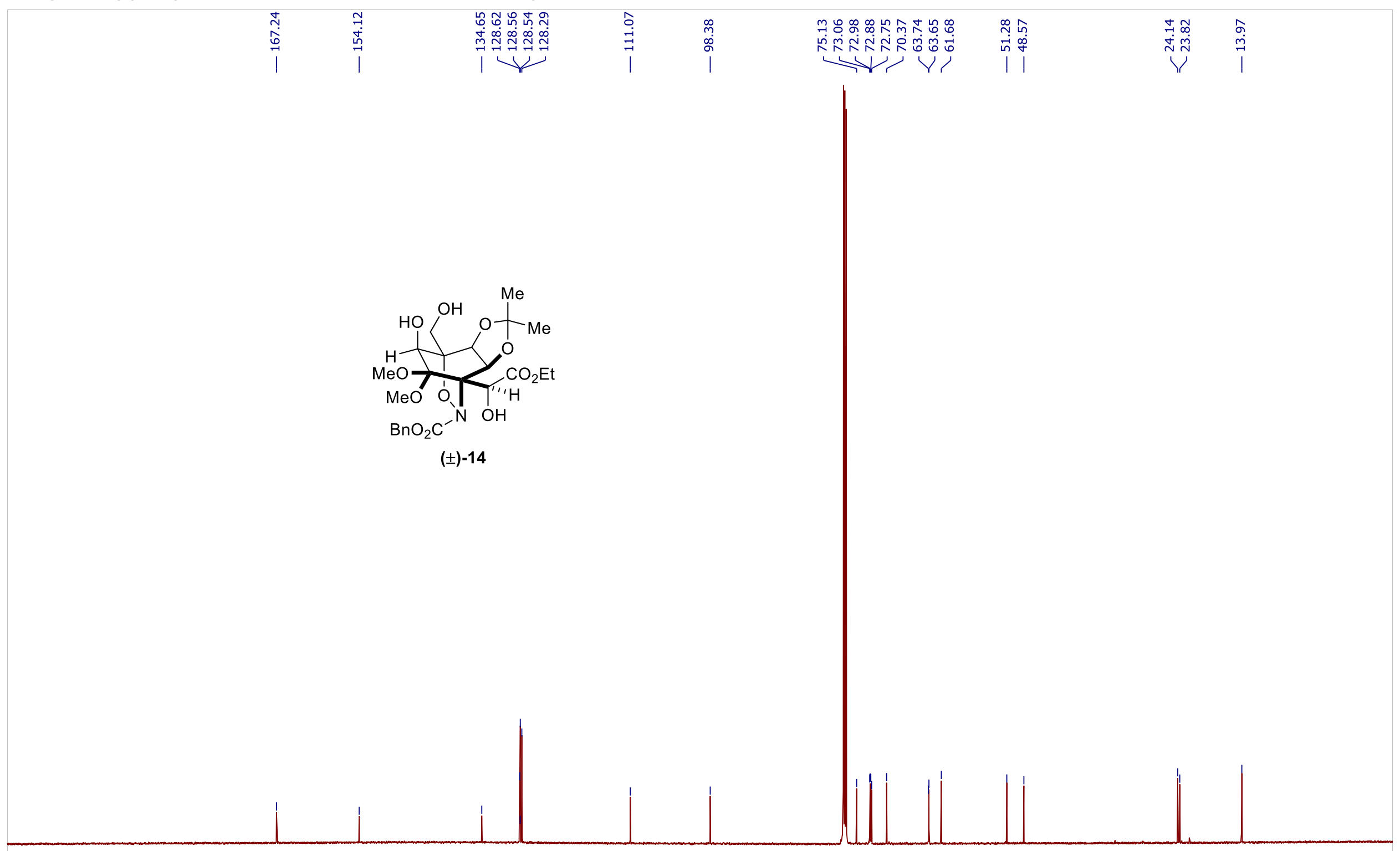

$10 \quad 200$

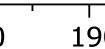

180

$170 \quad 160$

$150 \quad 140$

130

120

$110 \quad 100$ f1 (ppm) 
Selective 1D NOESY Experiments on ( \pm )-14

JGR20-TTX1-713 f2.11.fid

1D Selective Gradient NOESY

freq: $4.099 \mathrm{ppm}$

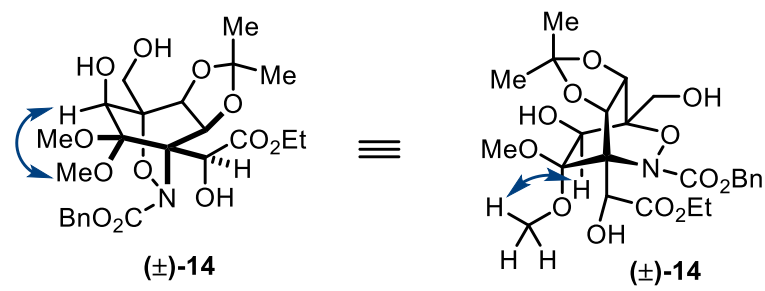

$( \pm)-14$

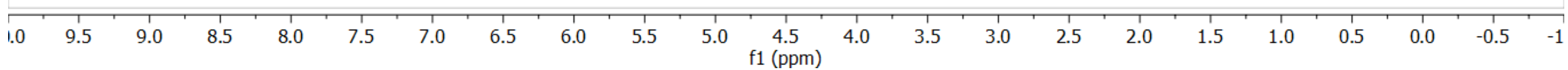




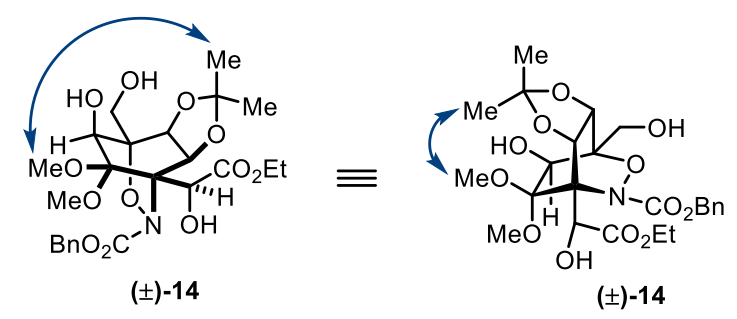

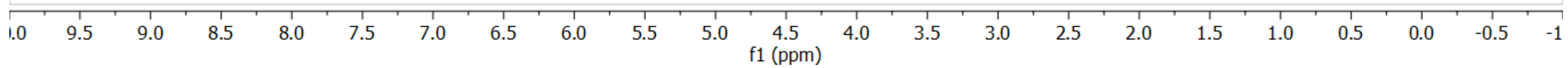



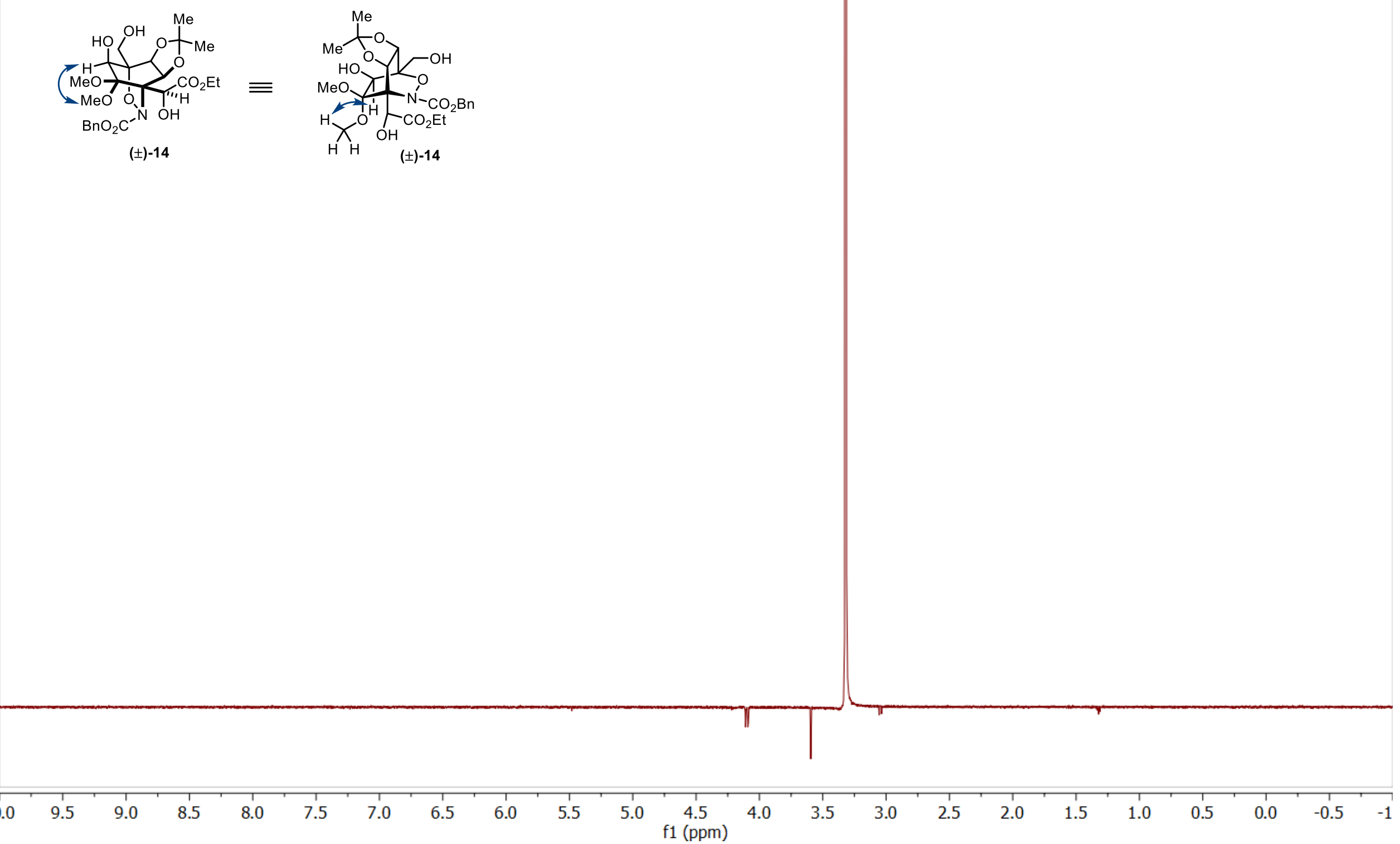
freq: $1.561 \mathrm{ppm}$

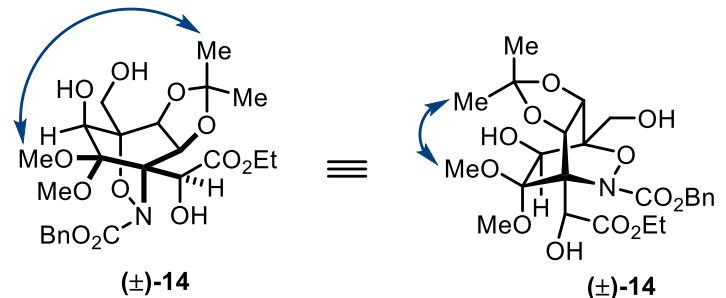

\begin{tabular}{|c|c|c|c|c|c|c|c|c|c|c|c|c|c|c|c|c|c|c|c|c|}
\hline 1.0 & 9.5 & 9.0 & 8.5 & 8.0 & 7.5 & 7.0 & 6.5 & 6.0 & 5.5 & 5.0 & \multicolumn{2}{|c|}{\begin{tabular}{l|l|l|} 
& 1 & 1 \\
4.5 & 4.0
\end{tabular}} & 3.5 & 3.0 & 2.5 & 2.0 & 1.5 & 1.0 & 0.5 & -0.5 \\
\hline
\end{tabular}


Compound ( \pm )-15 ( ${ }^{1} \mathrm{H}, 600 \mathrm{MHz}, \mathrm{CDCl}_{3}$, standard view)

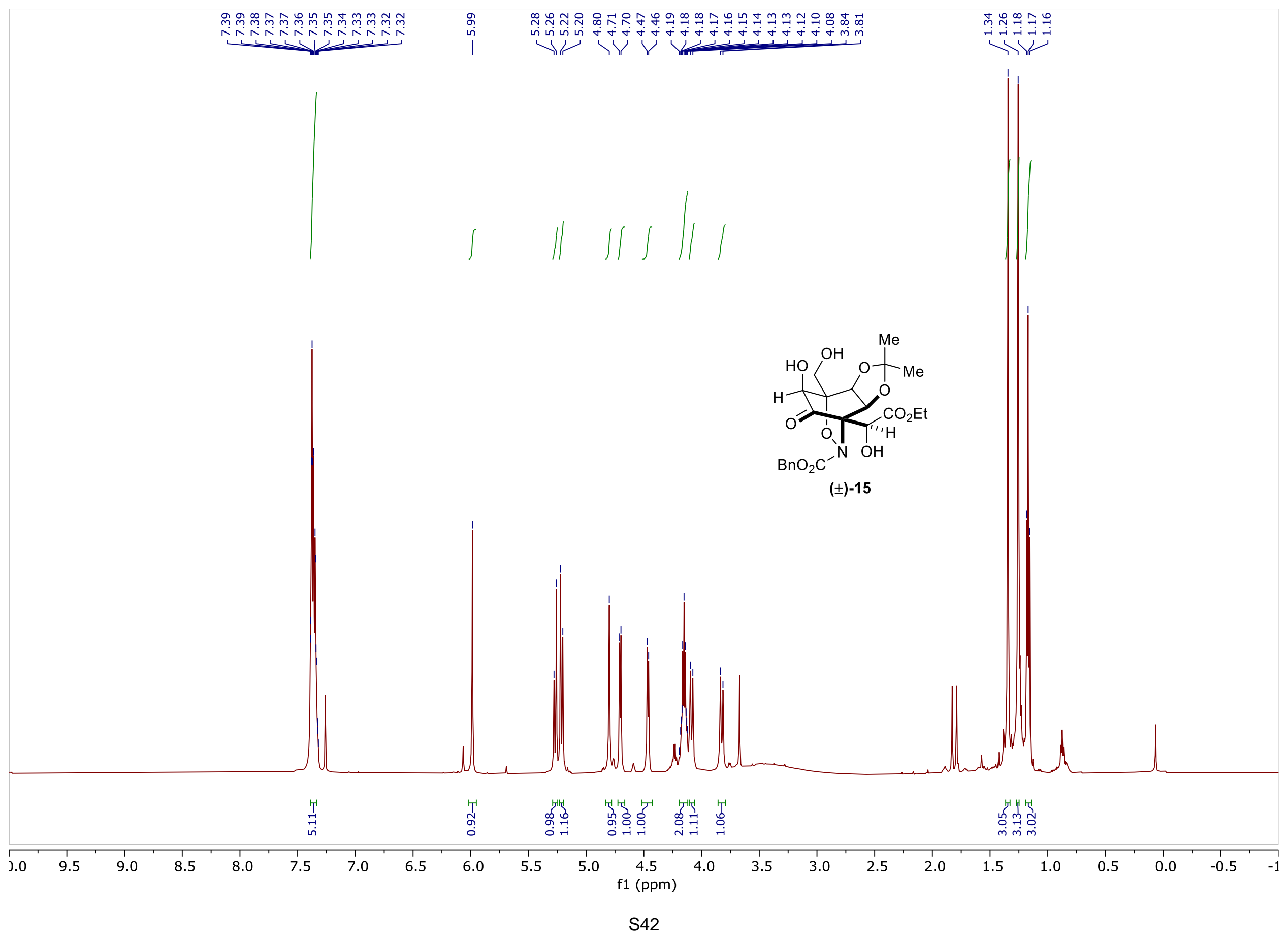


Compound ( \pm$)-15\left({ }^{13} \mathrm{C}\left\{{ }^{1} \mathrm{H}\right\}, 151 \mathrm{MHz}, \mathrm{CDCl}_{3}\right.$, standard view)
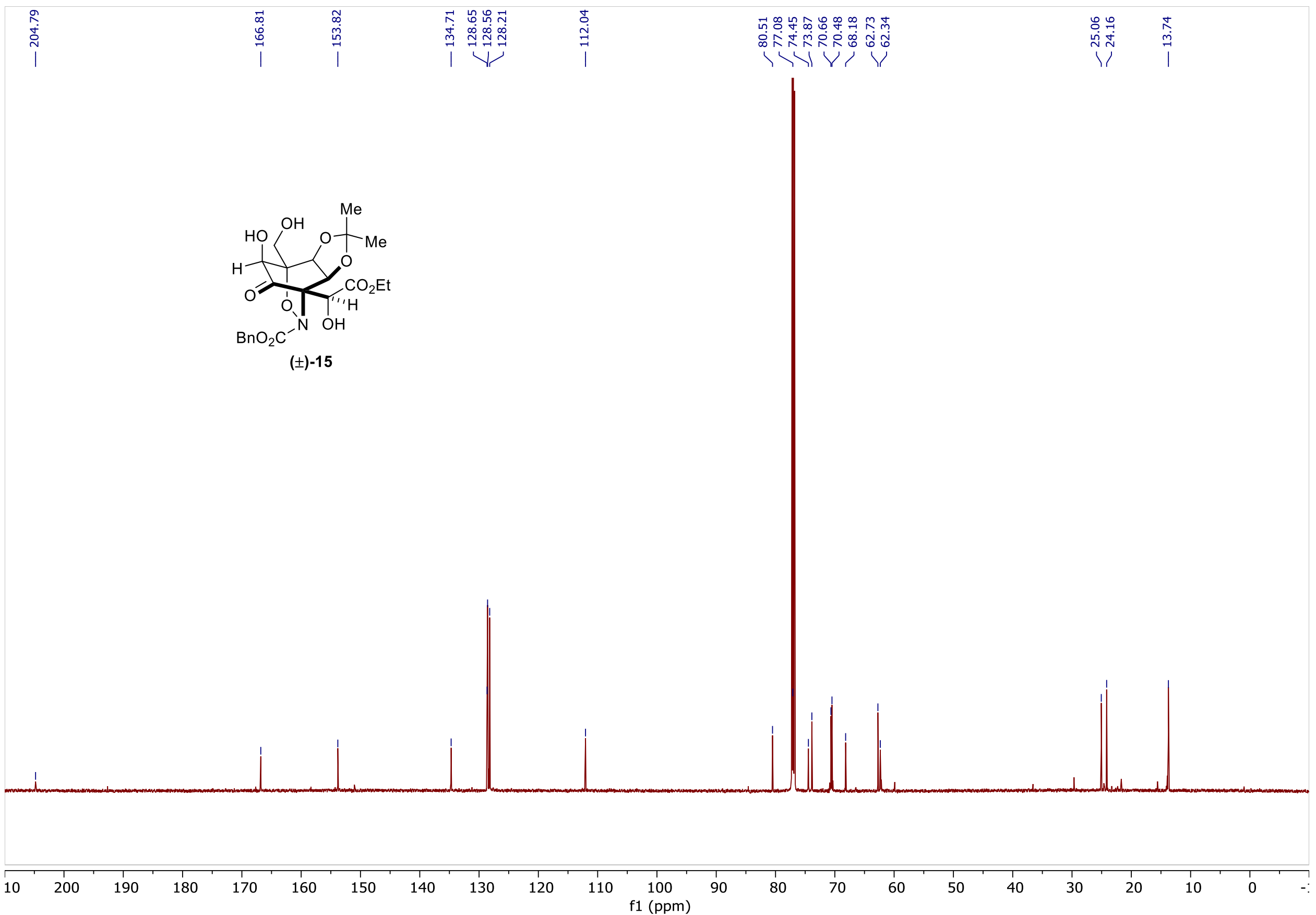


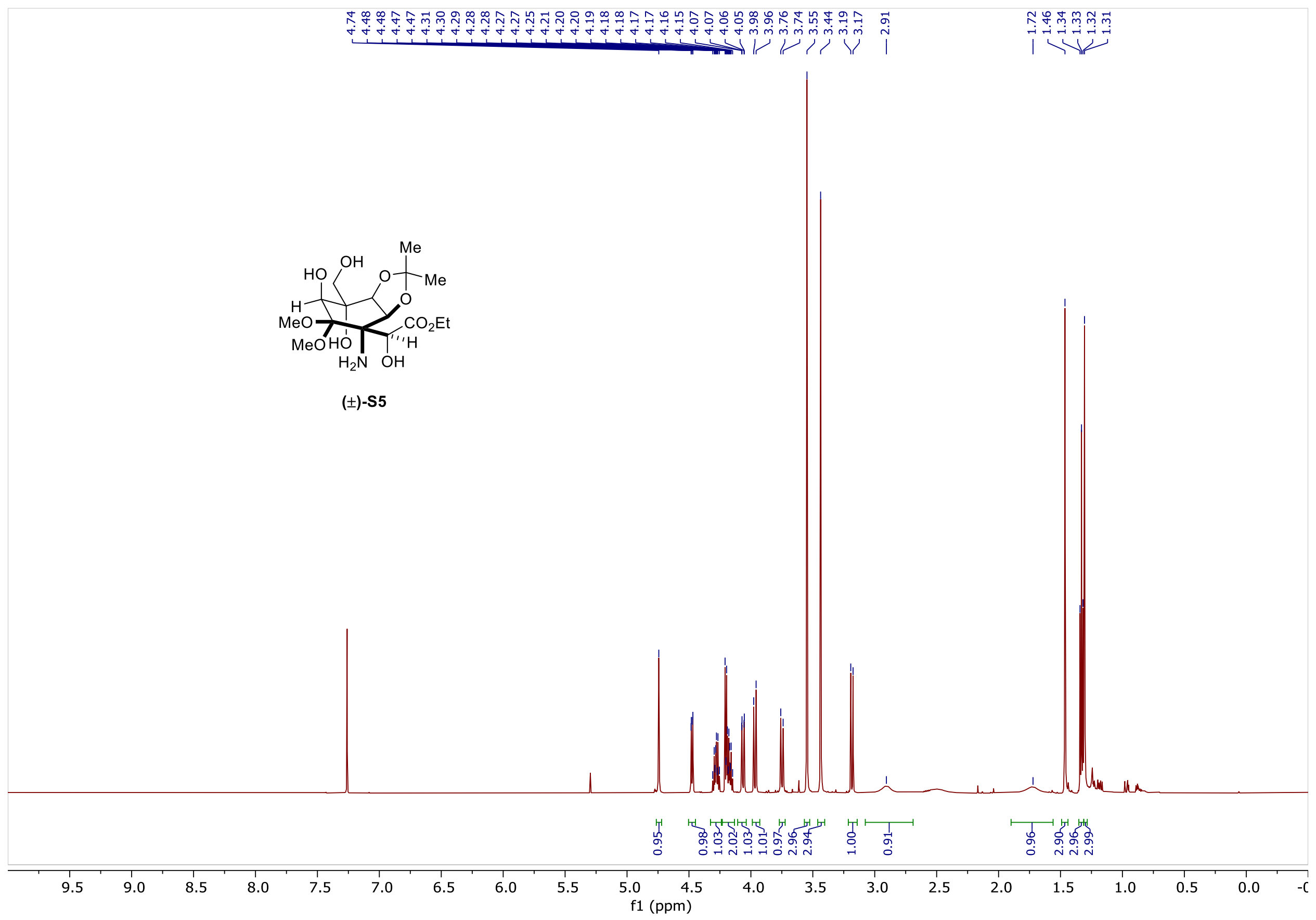


Compound ( \pm )-S5 $\left({ }^{13} \mathrm{C}\left\{{ }^{1} \mathrm{H}\right\}, 151 \mathrm{MHz}, \mathrm{CDCl}_{3}\right.$, standard view)

$\stackrel{\substack{\infty \\ \stackrel{N}{N}}}{\stackrel{1}{\mid}}$

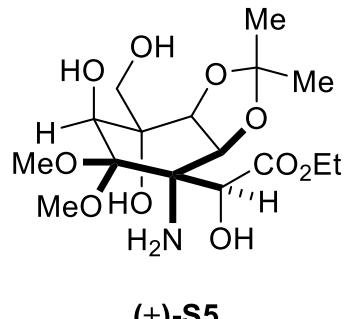

(土)-S5

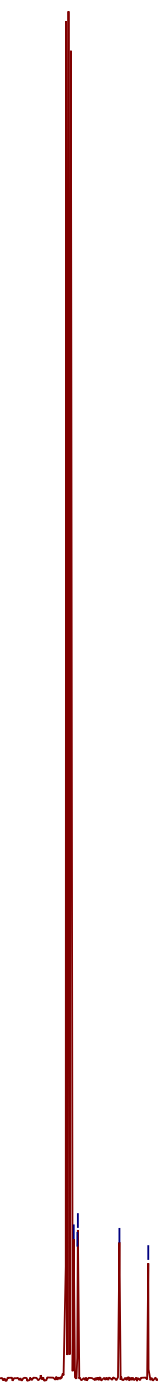




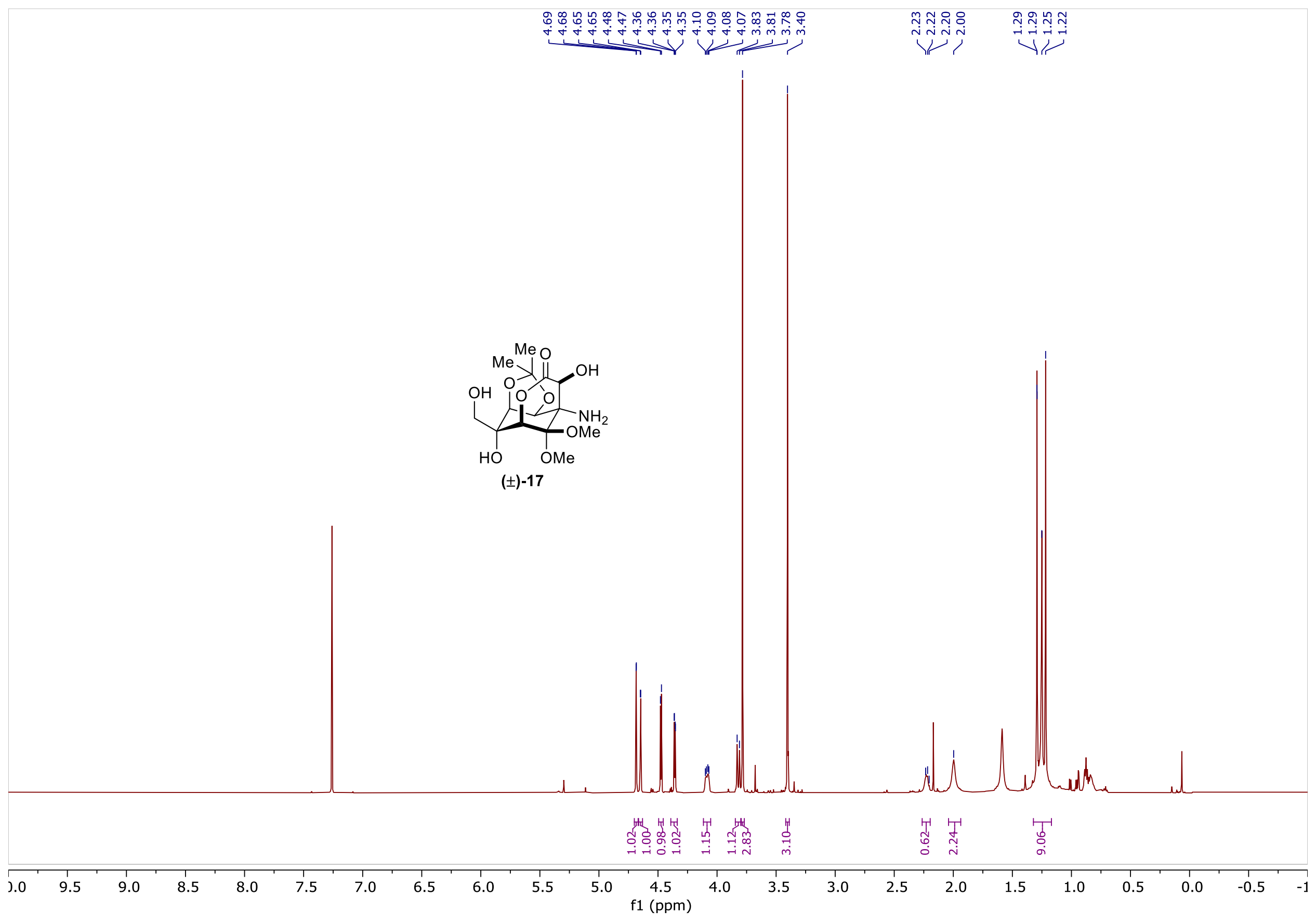


Compound ( \pm )-17 $\left({ }^{13} \mathrm{C}\left\{{ }^{1} \mathrm{H}\right\}, 151 \mathrm{MHz}, \mathrm{CDCl}_{3}\right.$, standard view)

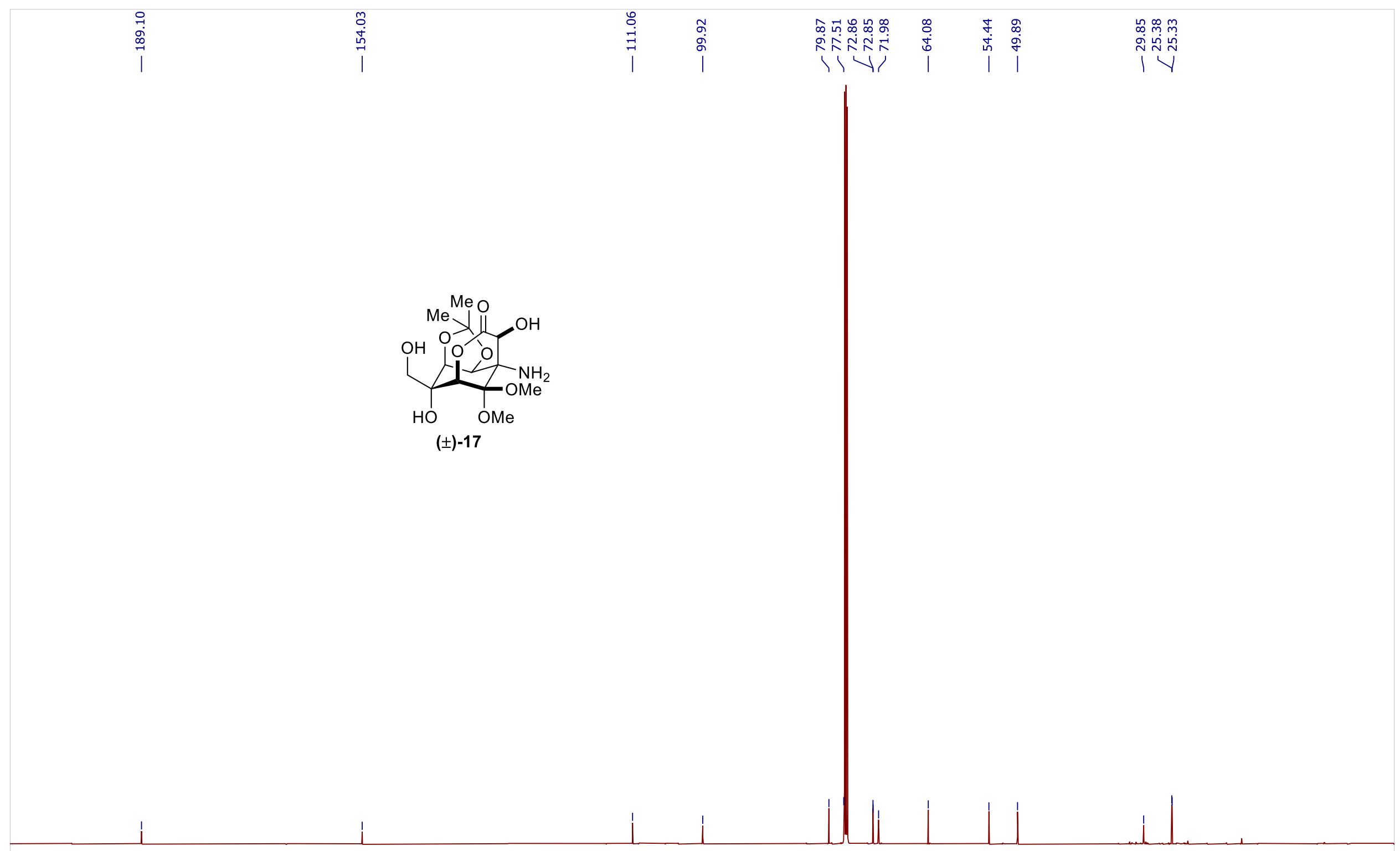

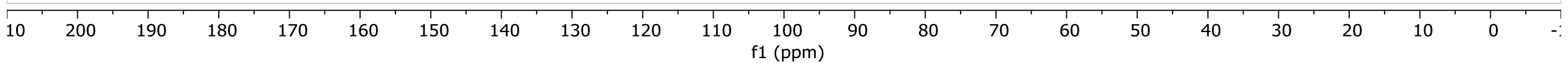


Compound ( \pm )-S6 ( ${ }^{1} \mathrm{H}, 600 \mathrm{MHz}, \mathrm{CDCl}_{3}$, standard view)

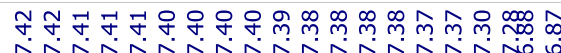

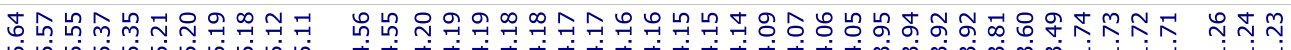

年

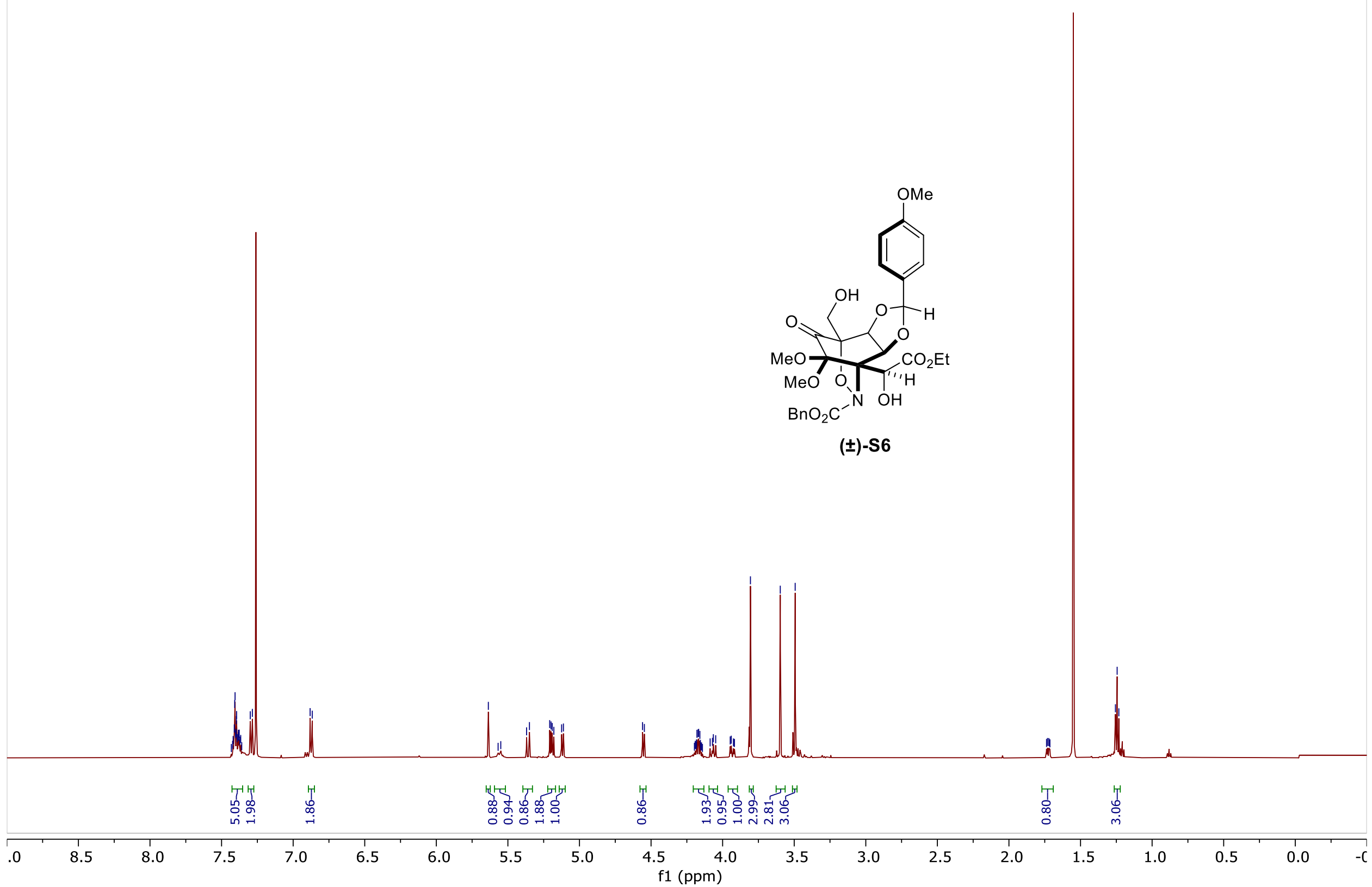


Compound $( \pm)-\mathrm{S} 6\left({ }^{13} \mathrm{C}\left\{{ }^{1} \mathrm{H}\right\}, 151 \mathrm{MHz}, \mathrm{CDCl}_{3}\right.$, standard view)

|

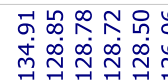

Y

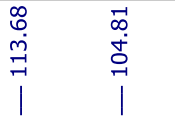

$\underset{\substack{0 \\ \infty \\ \infty}}{\substack{\infty \\ \mid}}$

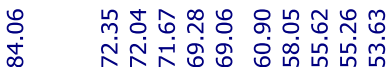

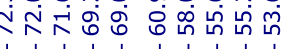

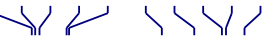

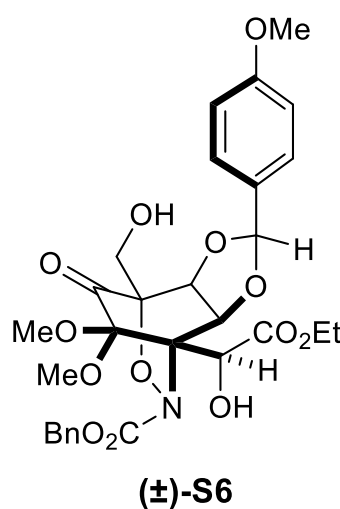

$( \pm)-S 6$

200

190

180

170

160

150

140

130

120

110

100
$1(\mathrm{ppm})$

1


Compound ( \pm )-20 ( ${ }^{1} \mathrm{H}, 600 \mathrm{MHz}, \mathrm{CDCl}_{3}$, standard view)

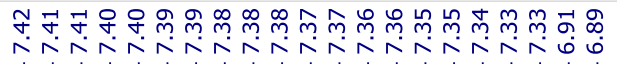

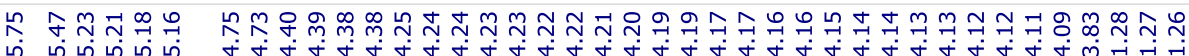

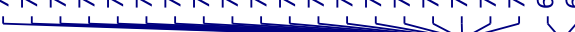

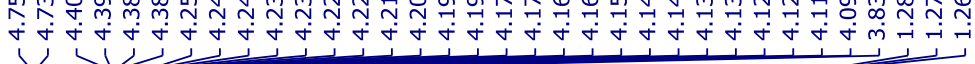

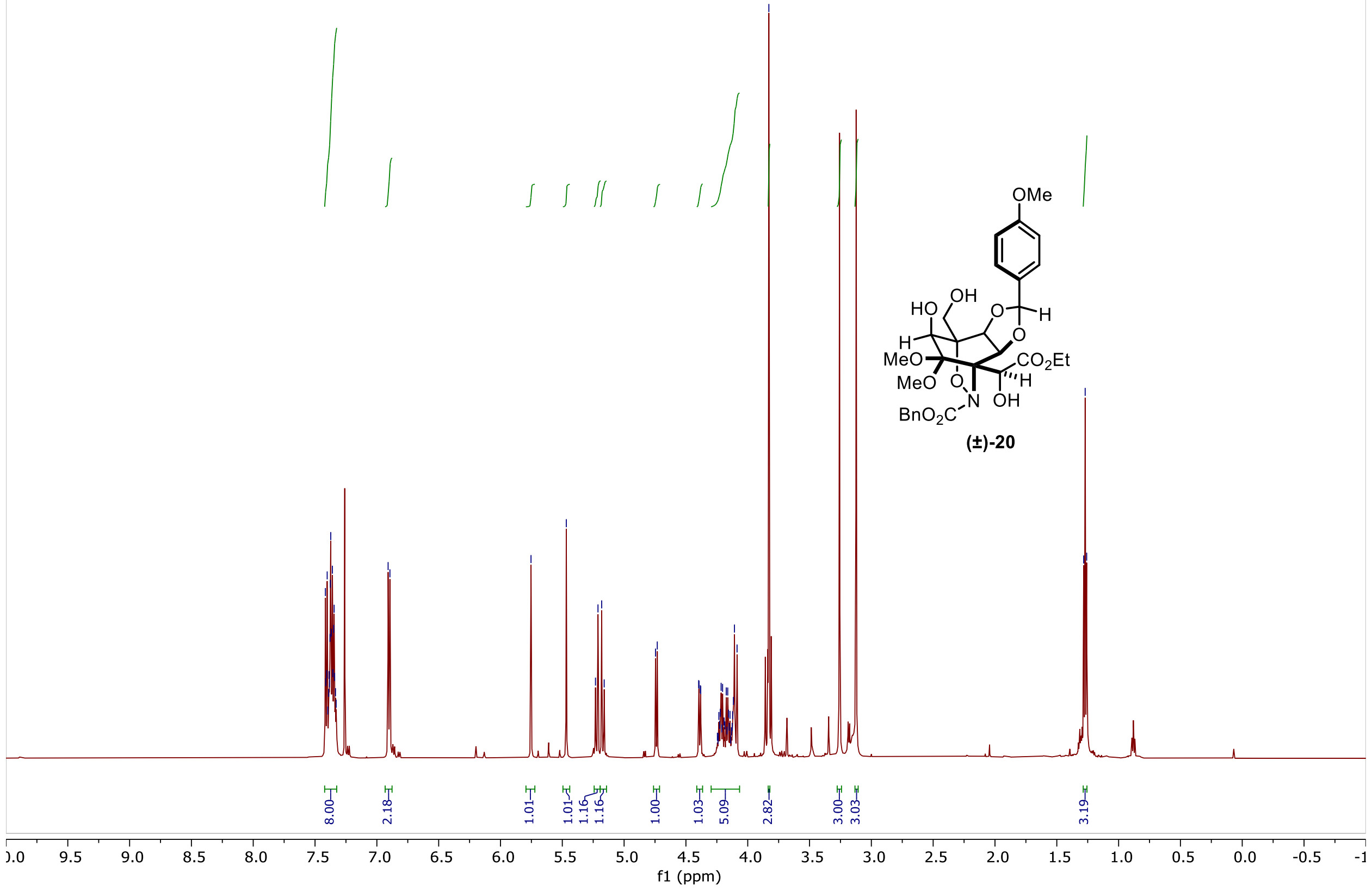


Compound $( \pm)-20\left({ }^{13} \mathrm{C}\left\{{ }^{1} \mathrm{H}\right\}, 151 \mathrm{MHz}, \mathrm{CDCl}_{3}\right.$, standard view)

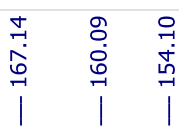

|

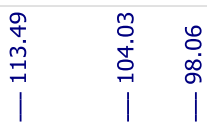

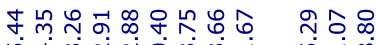

$\underbrace{\prime}$

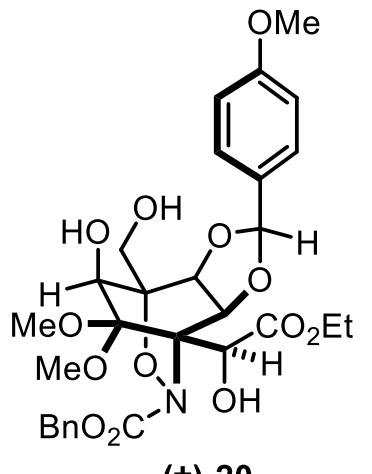

$( \pm)-20$

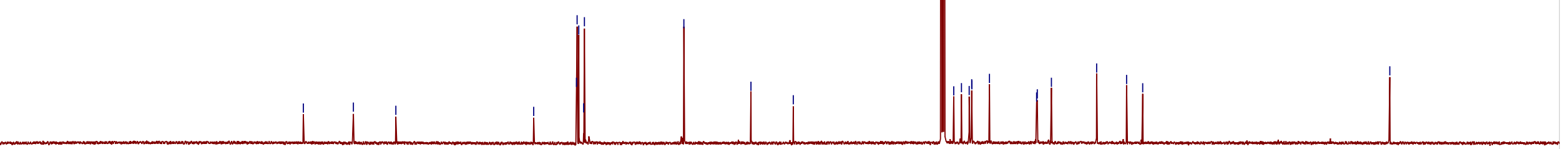




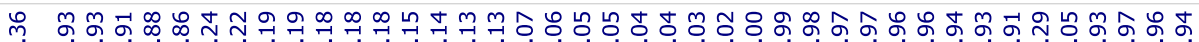

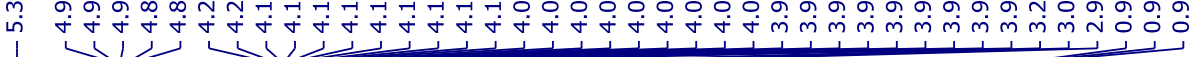

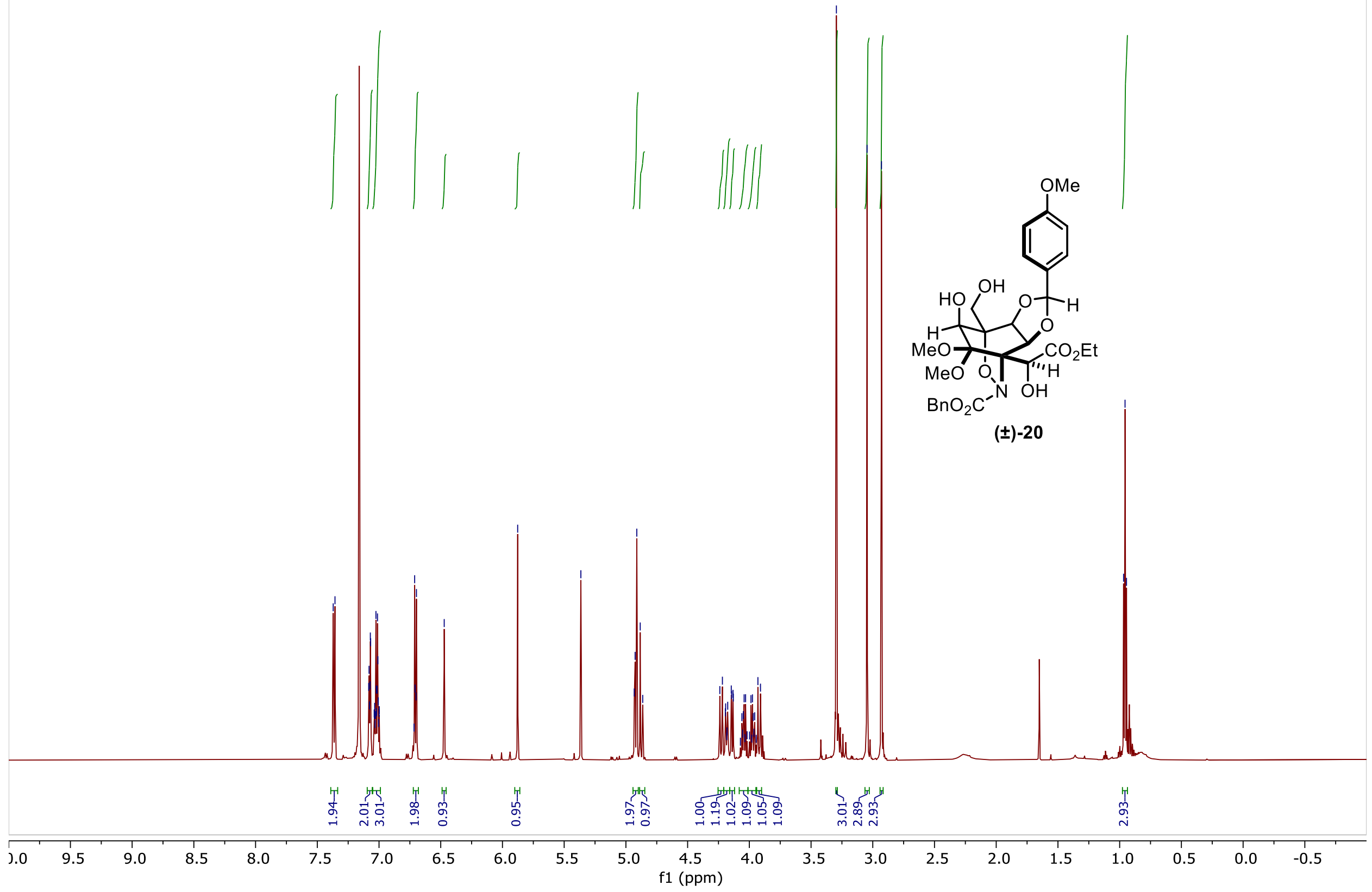


Compound $( \pm)-20\left({ }^{13} \mathrm{C}\left\{{ }^{1} \mathrm{H}\right\}, 151 \mathrm{MHz}, \mathrm{C}_{6} \mathrm{D}_{6}\right.$, standard view)

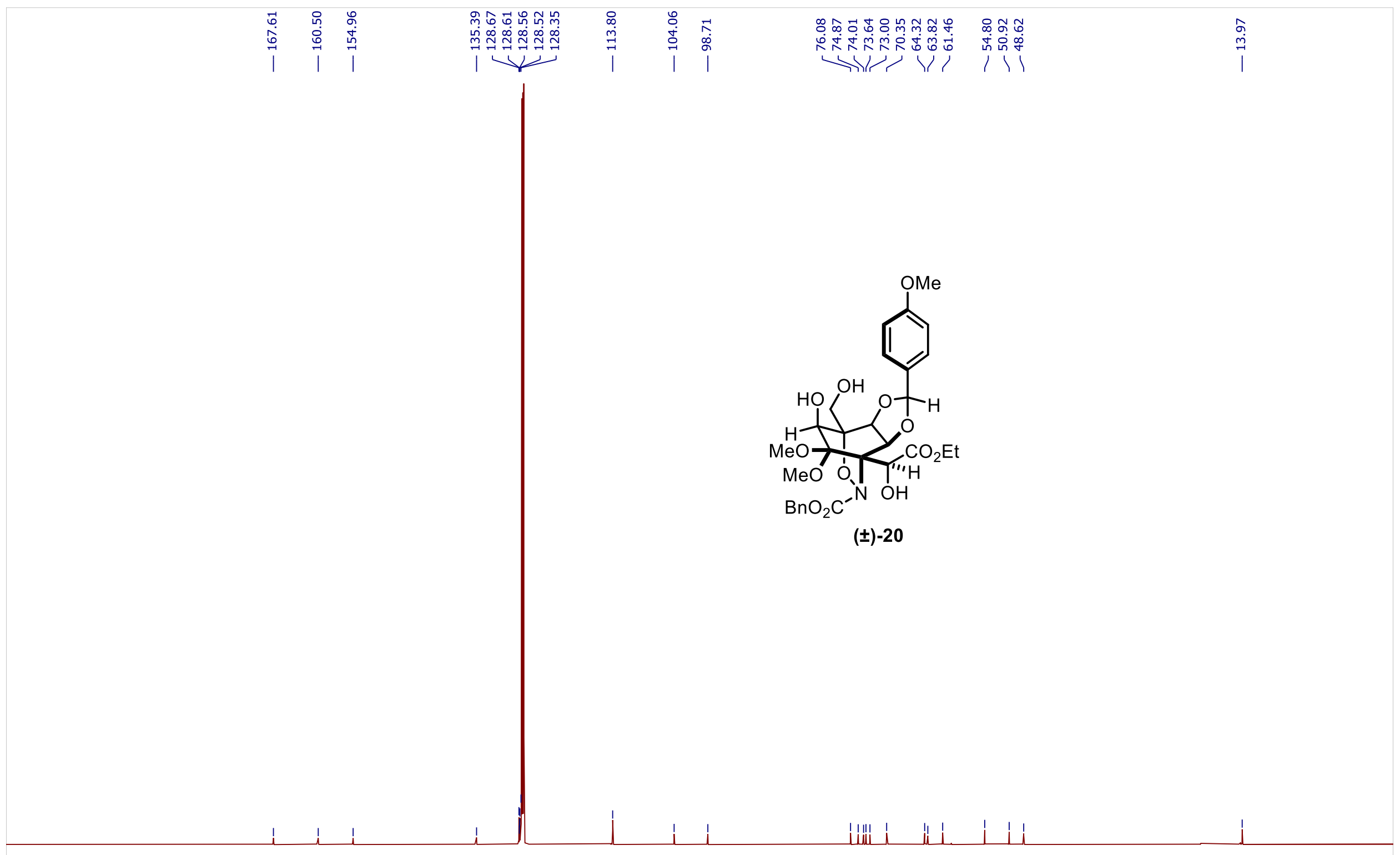

$10 \quad 200$

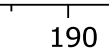

180

$170 \quad 160$

$150 \quad 140$

130

120

110100

90


HSQC NMR Spectrum of ( \pm )-20 in $C_{6} D_{6}$

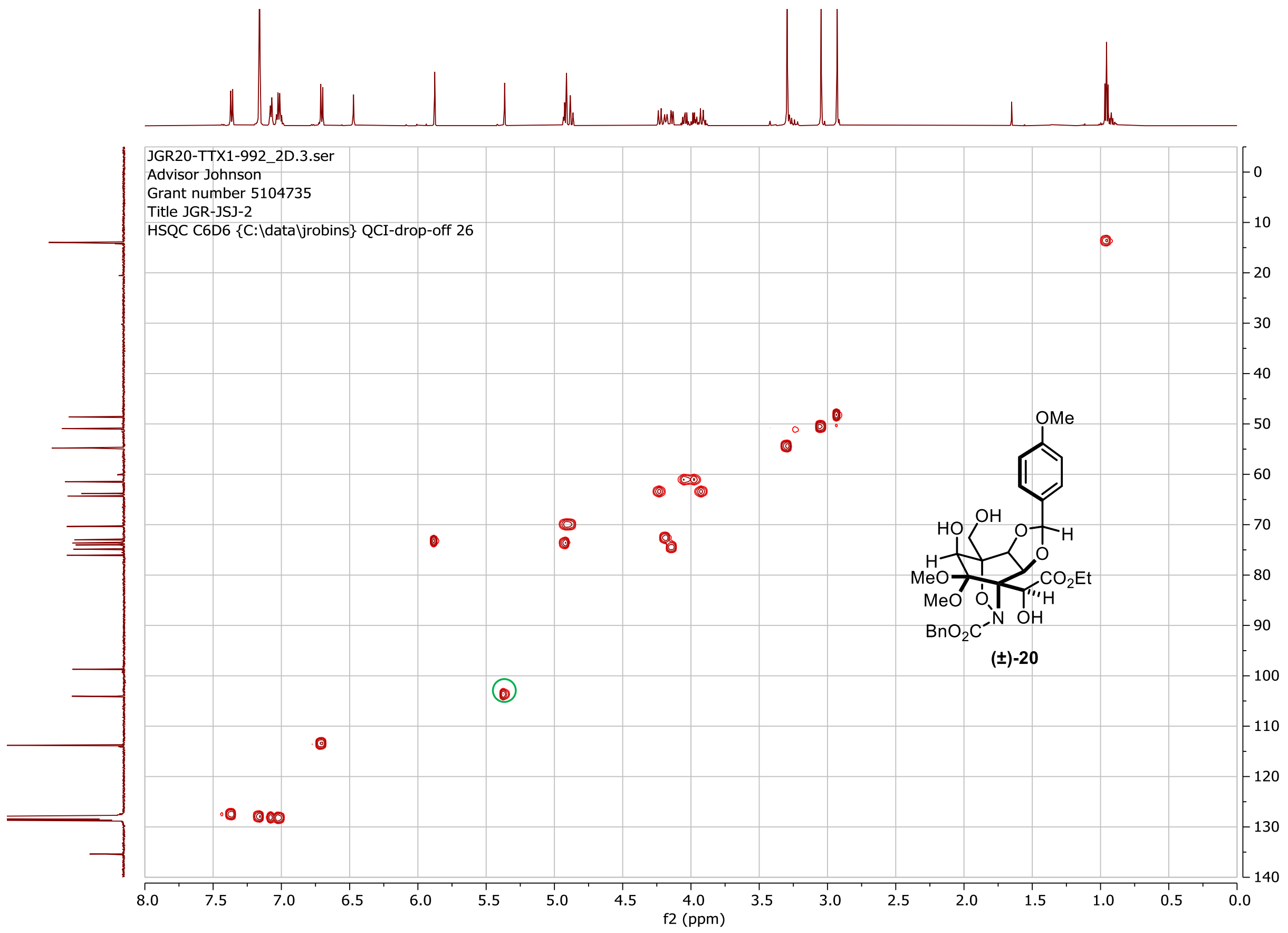


HMBC NMR Spectrum of ( \pm )-20 in $C_{6} D_{6}$

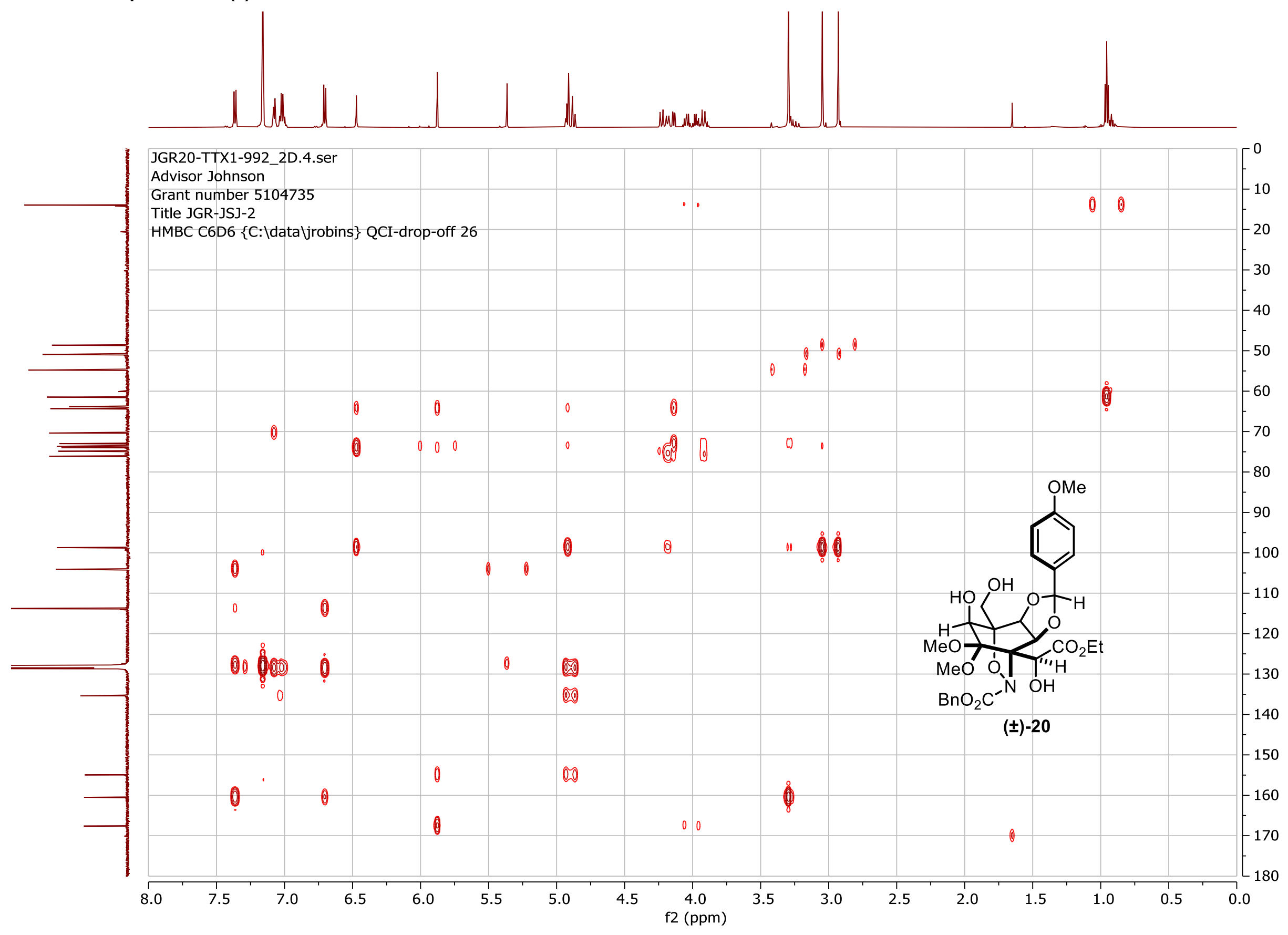


2D NOESY NMR Spectrum of ( \pm )-20 in $C_{6} D_{6}$

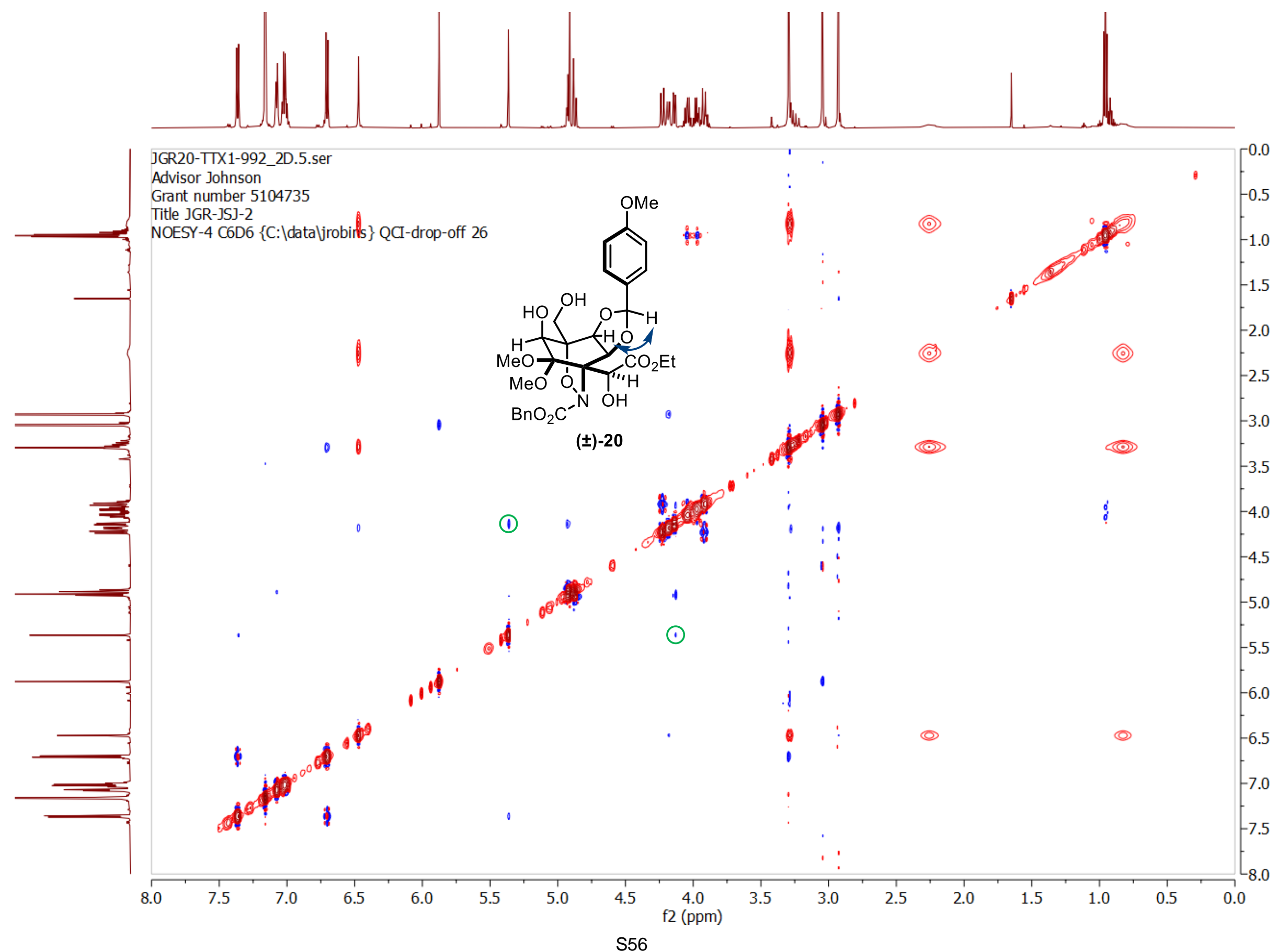


Compound ( \pm )-22 ( ${ }^{1} \mathrm{H}, 600 \mathrm{MHz}^{\mathrm{CDCl}} 3$, standard view)

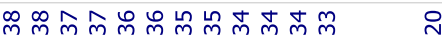

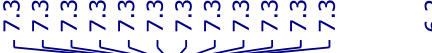

疋

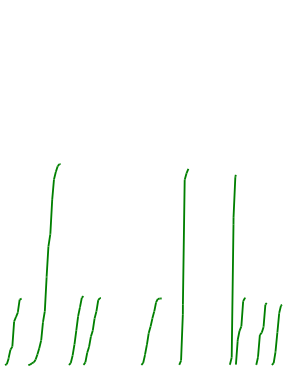

$( \pm)-22$

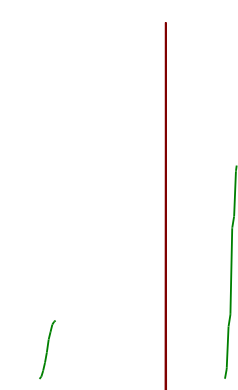

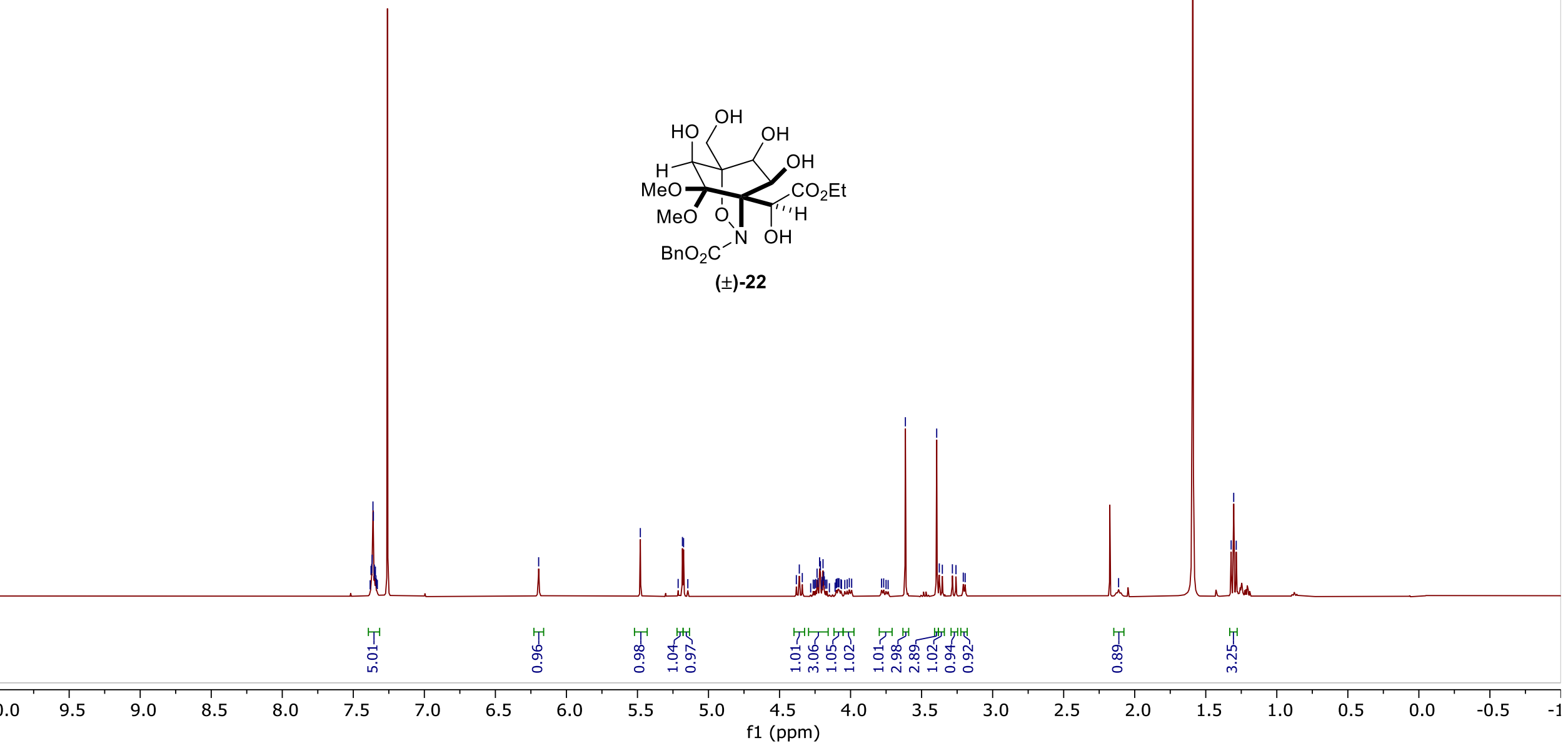

员 
Compound $( \pm)-22\left({ }^{13} \mathrm{C}\left\{{ }^{1} \mathrm{H}\right\}, 151 \mathrm{MHz}, \mathrm{CDCl}_{3}\right.$, standard view)

|

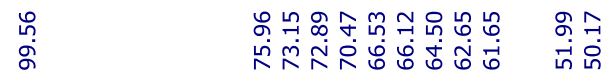

经)

$\stackrel{\substack{m \\ \grave{1}}}{\mid}$

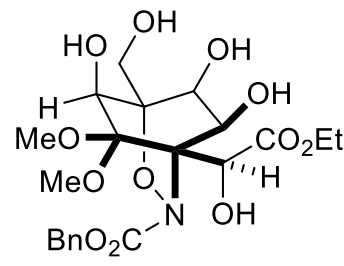

$( \pm)-22$ 


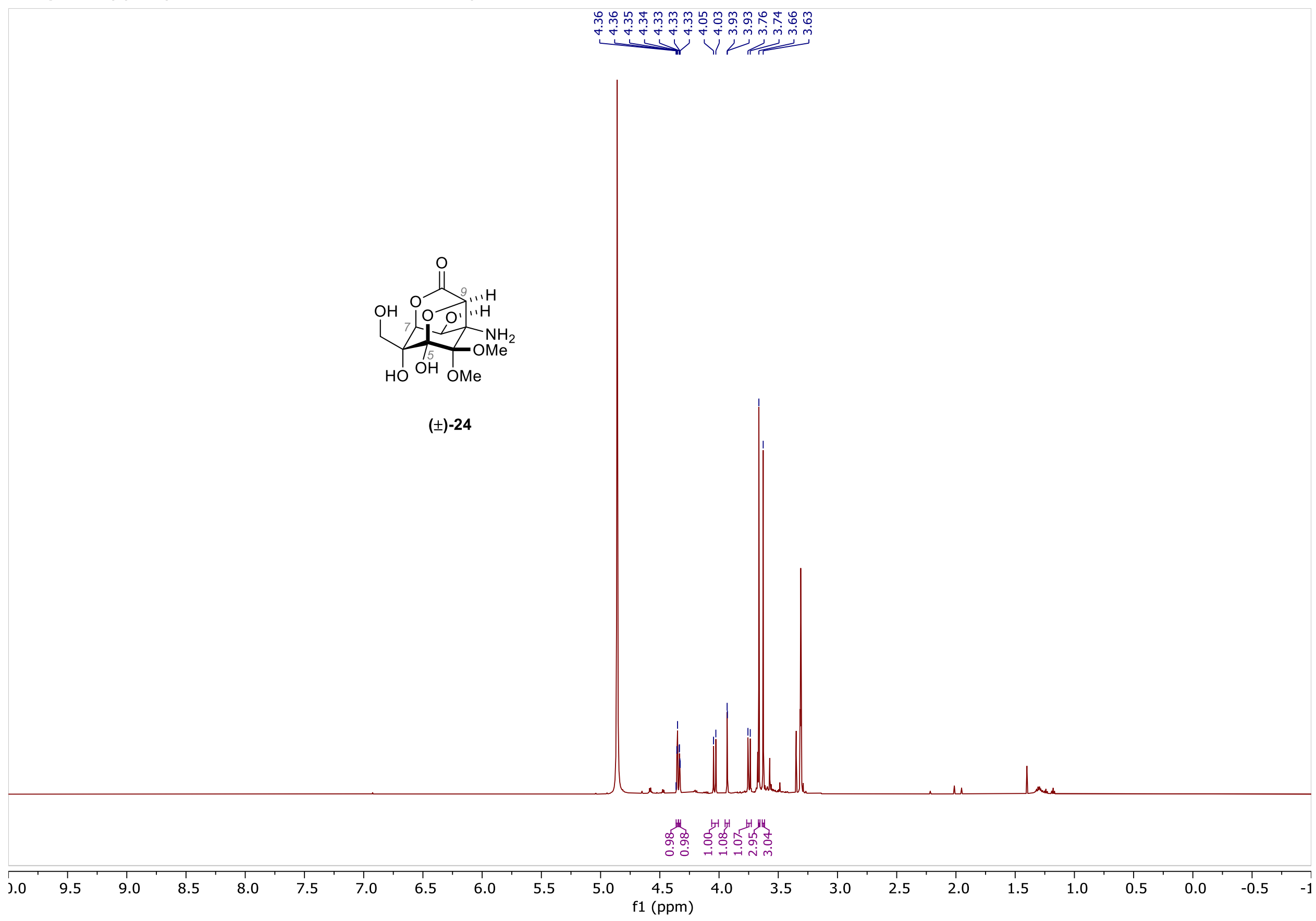


Compound ( \pm )-24 $\left({ }^{13} \mathrm{C}\left\{{ }^{1} \mathrm{H}\right\}, 151 \mathrm{MHz}, \mathrm{D}_{3} \mathrm{COD}\right.$, standard view)

至

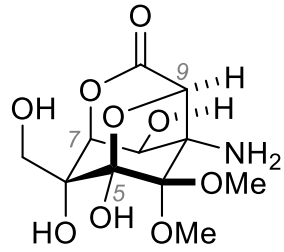

$( \pm)-24$

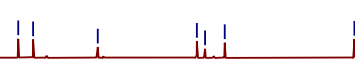


HPLC Data for Mandelate (-)-11

Sample preparation: Analyte solutions were prepared using HPLC grade solvents at concentrations ranging from $0.5 \mathrm{mg} / \mathrm{mL}$ to $1.5 \mathrm{mg} / \mathrm{mL}$. and the solutions were filtered through a $13 \mathrm{~mm}$ diameter syringe filter ( $0.45 \mu \mathrm{m}$ PTFE membrane).

\section{Compound 11}

JGR19-TTX1-590_IC_80:20:210:5:395:5 : 1

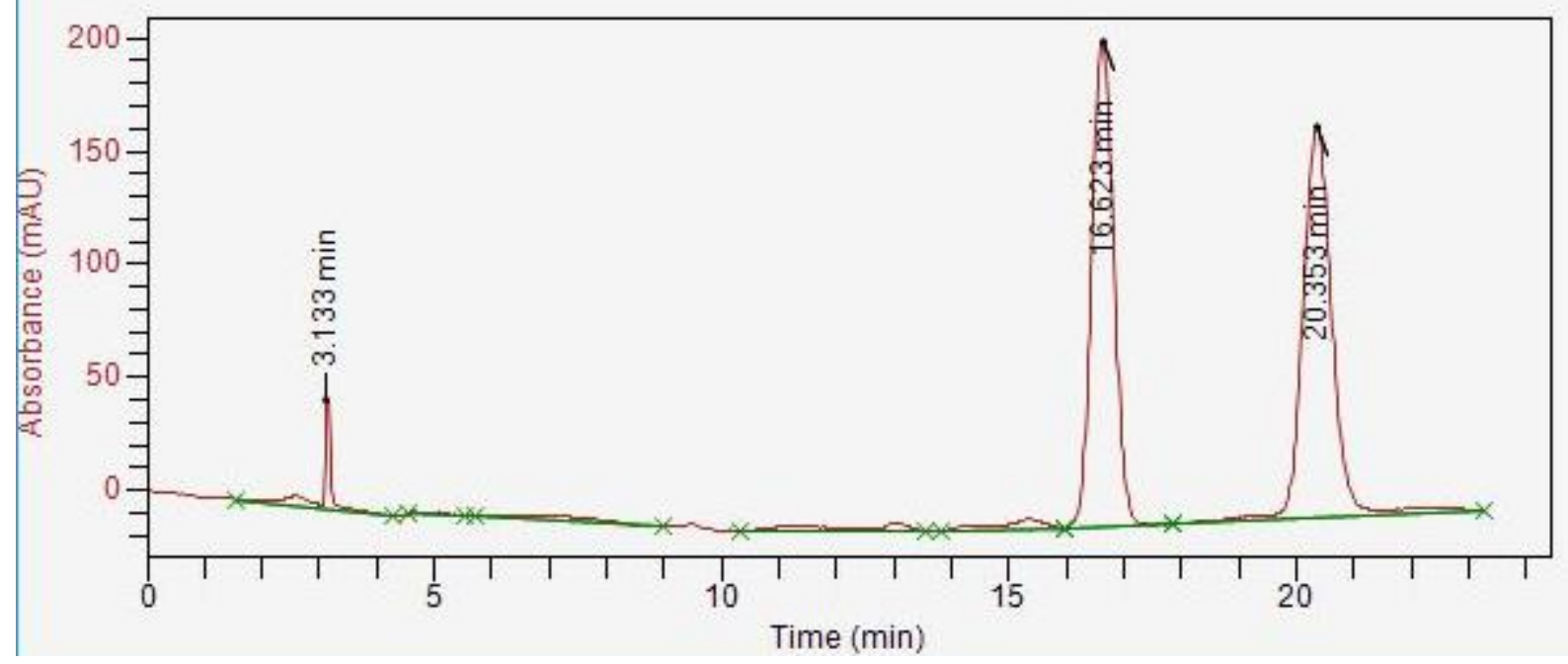

\begin{tabular}{lll}
\hline \hline $\begin{array}{l}\text { Retention Time } \\
(\mathrm{min})\end{array}$ & $\begin{array}{l}\text { Area } \\
(\mathrm{mAU} \bullet \mathrm{min})\end{array}$ & Area \% \\
\hline \hline 16.625 & 5619789.17 & 47.5 \\
20.353 & 6211643.36 & 52.5
\end{tabular}

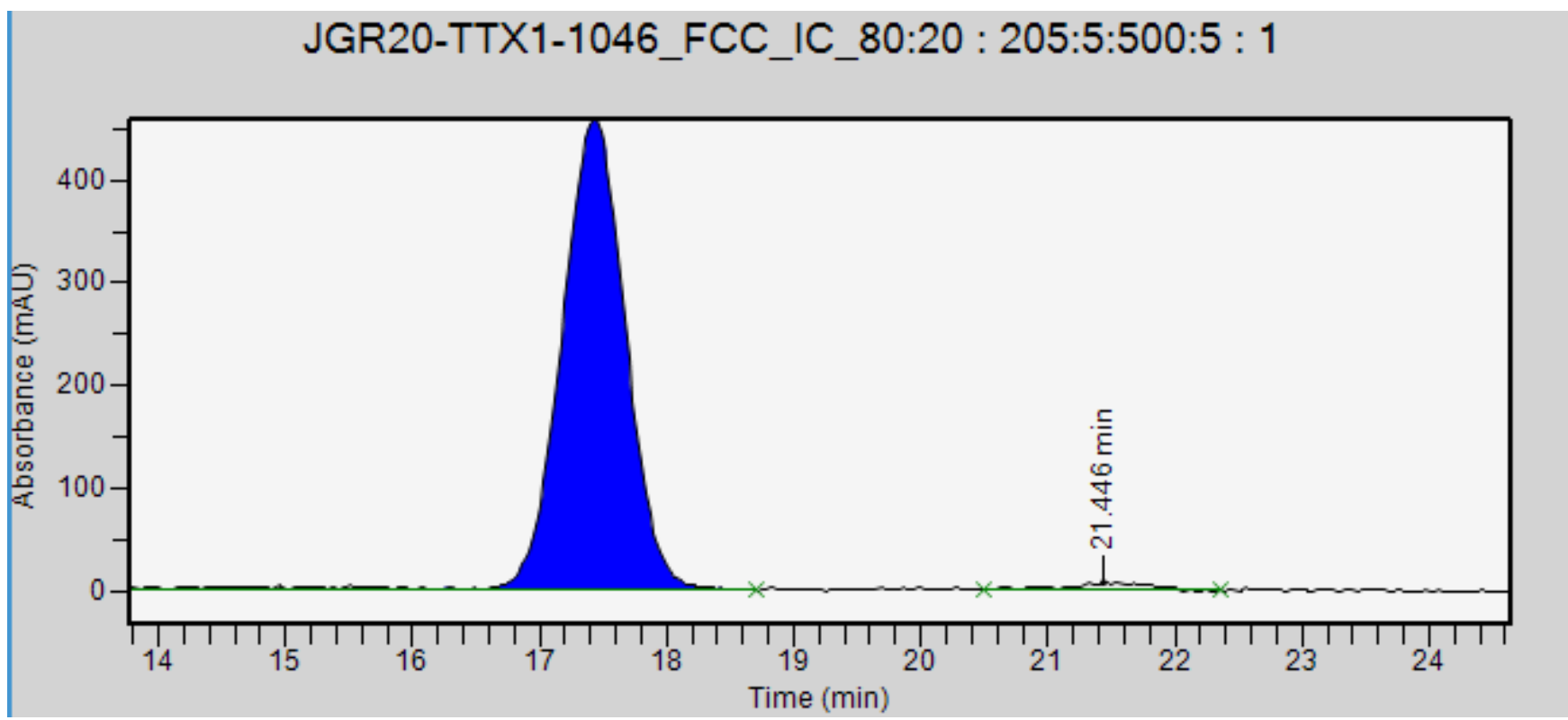

\begin{tabular}{lll}
\hline \hline $\begin{array}{l}\text { Retention Time } \\
(\mathrm{min})\end{array}$ & $\begin{array}{l}\text { Area } \\
(\mathrm{mAU} \bullet \min )\end{array}$ & Area \% \\
\hline \hline 17.431 & 15836754.22 & 98.5 \\
21.446 & 246342.12 & 1.5
\end{tabular}




\section{X-Ray Diffraction Data for Compound 24}

Sample preparation: A 1-dram vial was charged with 15 milligrams of compound 24 and $0.15 \mathrm{~mL}$ of methanol. The vial was placed on a scintillation vial containing $10 \mathrm{~mL}$ of diethyl ether, capped, and the compound was allowed to crystallize using the method of vapor diffusion over three days.

Data collection: A colorless crystal (approximate dimensions $0.070 \times 0.020 \times 0.020 \mathrm{~mm}^{3}$ ) was placed onto the tip of MiTeGen and mounted on a Bruker D8 VENTURE diffractometer and measured at $100 \mathrm{~K}$.

A preliminary set of cell constants was calculated from reflections harvested from three sets of 12 frames. These initial sets of frames were oriented such that orthogonal wedges of reciprocal space were surveyed. This produced initial orientation matrices determined from 350 reflections. The data collection was carried out using $\mathrm{Cu} \mathrm{Ka}$ radiation (graphite monochromator) with a frame time of 2 seconds and a detector distance of $5.0 \mathrm{~cm}$. A randomly oriented region of reciprocal space was surveyed to achieve complete data with a redundancy of 4 . Sections of frames were collected with $0.50^{\circ}$ steps in $\omega$ and $\square$ scans. Data to a resolution of $0.71 \AA$ were considered in the reduction. Final cell constants were calculated from the xyz centroids of 9244 strong reflections from the actual data collection after integration (SAINT). The intensity data were corrected for absorption (SADABS). Please refer to Table 1 for additional crystal and refinement information.

Structure solution and refinement: The space group $P 2_{1} / n$ was determined based on intensity statistics and systematic absences. The structure was solved using Superflip and refined (full-matrix-least squares) using the Oxford University Crystals for Windows system. The charge-flipping solution provided most non-hydrogen atoms from the E-map. Full-matrix least squares / difference Fourier cycles were performed, which located the remaining non-hydrogen atoms. All nonhydrogen atoms were refined with anisotropic displacement parameters. The hydrogen atoms were placed in ideal positions and refined with individual relative isotropic displacement parameters. The final full matrix least squares refinement converged to $\mathrm{R} 1=0.0462$ and $w R 2=0.1172\left(\mathrm{~F}^{2}\right.$, all data).

Structure description: The structure was found as proposed. 


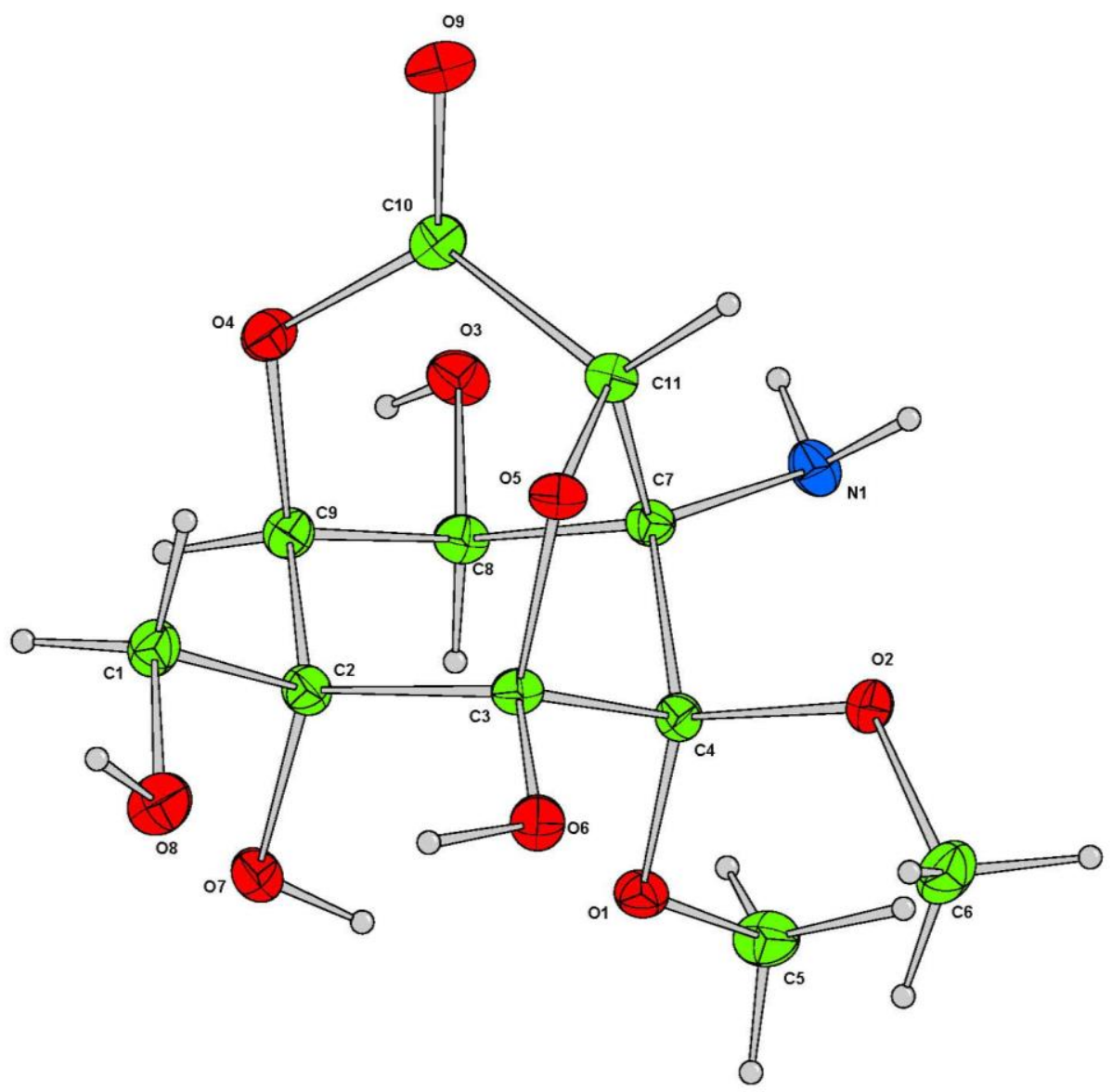

Molecular structure with labels on the asymmetric unit 


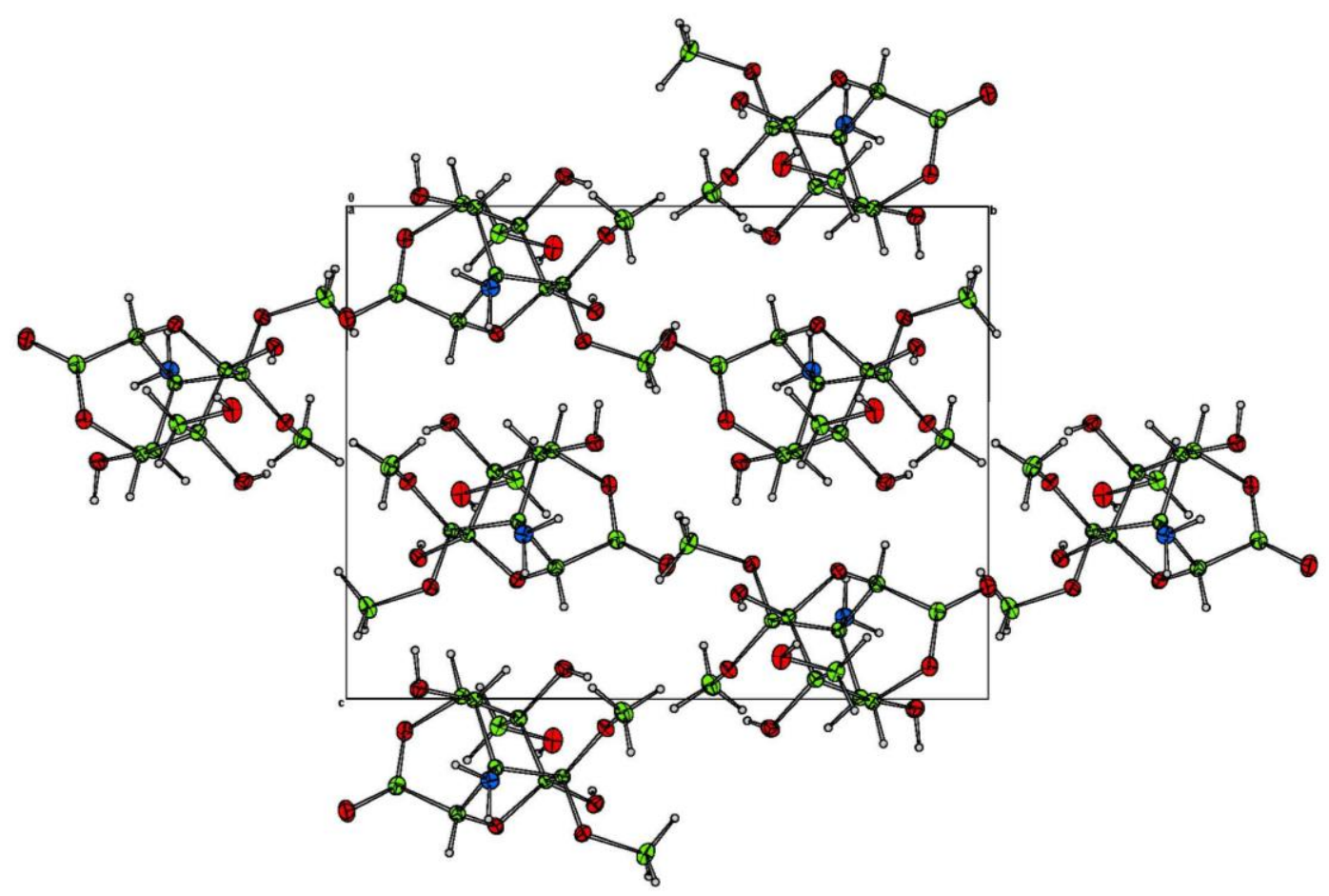

Cell plot, viewed along a- axis

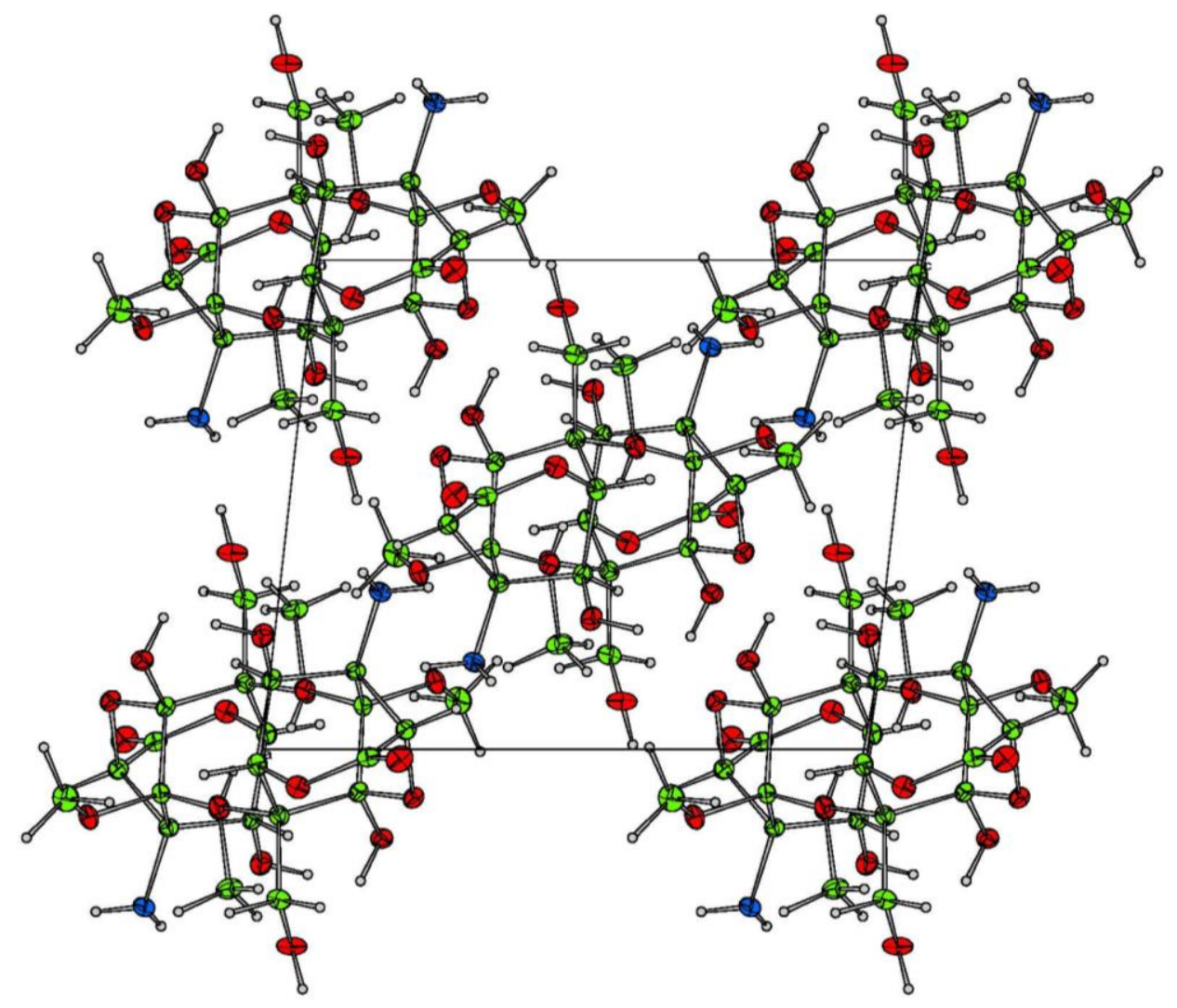

Cell plot, viewed along $b$ - axis 


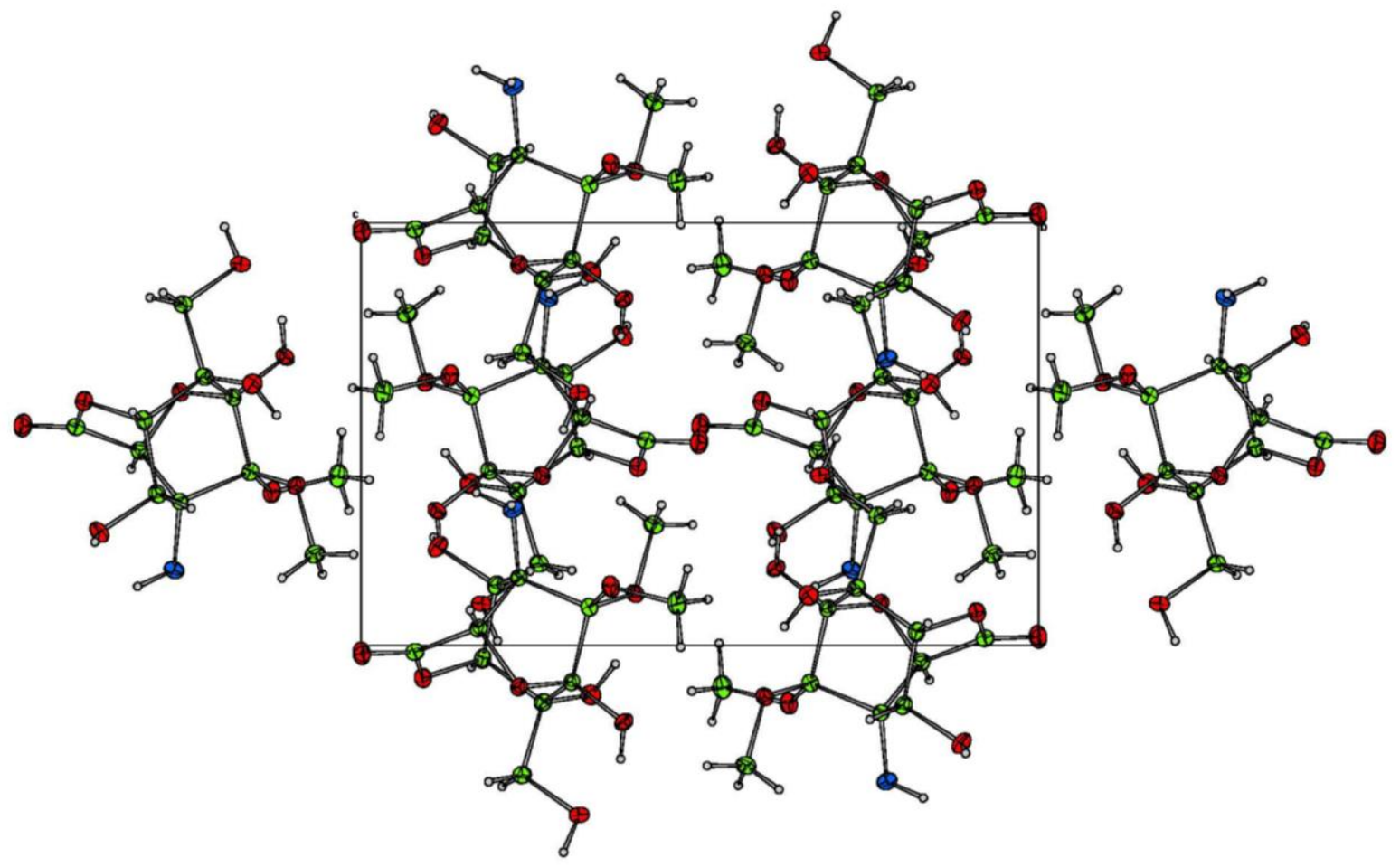

Cell plot, viewed along c- axis 
Table 1. Crystal data and structure refinement for $\mathbf{S} 19177$.

Empirical formula

Formula weight

Crystal color, shape, size

Temperature

Wavelength

Crystal system, space group

Unit cell dimensions

Volume

Z

Density (calculated)

Absorption coefficient

$\mathrm{F}(000)$

Data collection

Diffractometer

Theta range for data collection

Index ranges

Reflections collected

Independent reflections

Observed Reflections

Completeness to theta $=27.531^{\circ}$

Solution and Refinement

Absorption correction

Max. and min. transmission

Solution

Refinement method

Weighting scheme

Data / restraints / parameters

Goodness-of-fit on $\mathrm{F}^{2}$

Final $R$ indices [l>2sigma(I)]

$R$ indices (all data)

Largest diff. peak and hole
C11 H17 N1 O9

307.26

colorless acicular, $0.070 \times 0.020 \times 0.020 \mathrm{~mm}^{3}$

$100 \mathrm{~K}$

$0.71073 \AA$

Monoclinic, $P 121 / \mathrm{n} 1$

$a=8.5747(10) \AA \quad \alpha=90^{\circ}$.

$\mathrm{b}=13.6821(11) \AA \quad B=96.341(4)^{\circ}$.

$\mathrm{c}=10.5583(11) \AA \quad \mathrm{V}=90^{\circ}$.

1231.1(2) $\AA^{3}$

4

$1.658 \mathrm{Mg} / \mathrm{m}^{3}$

$0.146 \mathrm{~mm}^{-1}$

648

Bruker Apex Kappa Duo, Bruker

2.446 to $35.296^{\circ}$.

$-12<=\mathrm{h}<=13,-21<=\mathrm{k}<=19,-17<=\mathrm{k}<=15$

20966

$4971[R$ (int) $=0.054]$

3305

$99.6 \%$

Semi-empirical from equivalents

1.00 and 1.00

Charge-Flipping methods

Full-matrix least-squares on $\mathrm{F}^{2}$

$w=\left[\sigma^{2} \mathrm{Fo}^{2}+\mathrm{AP}^{2}+\mathrm{BP}\right]^{-1}$, with

$\mathrm{P}=\left(\mathrm{Fo}^{2}+2 \mathrm{Fc}^{2}\right) / 3, \mathrm{~A}=0.049, \mathrm{~B}=0.420$

4963 / 14 / 247

0.9778

$\mathrm{R} 1=0.0462, \mathrm{wR} 2=0.1017$

$\mathrm{R} 1=0.0815, \mathrm{wR} 2=0.1172$

0.80 and -0.70 e. $\AA^{-3}$ 
Table 2. Atomic coordinates $\left(\times 10^{4}\right)$ and equivalent isotropic displacement parameters $\left(\AA^{2} \times 10^{3}\right)$ for $\mathrm{S} 19177$. $\mathrm{U}(\mathrm{eq})$ is defined as one third of the trace of the orthogonalized $\mathrm{U}^{\mathrm{ij}}$ tensor.

\begin{tabular}{lrrrr}
\hline & $\mathrm{x}$ & $\mathrm{y}$ & $\mathrm{z}$ & $\mathrm{U}(\mathrm{eq})$ \\
\hline O1 & $6222(1)$ & $9043(1)$ & $4405(1)$ & 13 \\
O2 & $6382(1)$ & $8687(1)$ & $2261(1)$ & 15 \\
O3 & $7326(1)$ & $6136(1)$ & $5196(1)$ & 16 \\
O4 & $4227(1)$ & $5913(1)$ & $4339(1)$ & 15 \\
O5 & $4012(1)$ & $7332(1)$ & $2411(1)$ & 13 \\
O6 & $3180(1)$ & $8879(1)$ & $2860(1)$ & 14 \\
O7 & $3823(1)$ & $8394(1)$ & $5602(1)$ & 15 \\
O8 & $981(1)$ & $8219(1)$ & $4149(1)$ & 19 \\
O9 & $4802(1)$ & $5009(1)$ & $2712(1)$ & 19 \\
N1 & $8218(1)$ & $7241(1)$ & $3339(1)$ & 14 \\
C1 & $1930(2)$ & $7375(1)$ & $4443(1)$ & 15 \\
C2 & $3648(2)$ & $7688(1)$ & $4601(1)$ & 13 \\
C3 & $4130(2)$ & $8112(1)$ & $3329(1)$ & 11 \\
C4 & $5895(2)$ & $8367(1)$ & $3412(1)$ & 12 \\
C5 & $7848(2)$ & $9320(1)$ & $4717(1)$ & 17 \\
C6 & $5996(2)$ & $9667(1)$ & $1850(2)$ & 20 \\
C7 & $6600(2)$ & $7327(1)$ & $3600(1)$ & 11 \\
C8 & $6424(2)$ & $6994(1)$ & $4970(1)$ & 13 \\
C9 & $4682(2)$ & $6799(1)$ & $5046(1)$ & 13 \\
C10 & $4838(2)$ & $5794(1)$ & $3228(1)$ & 14 \\
C11 & $5402(2)$ & $6735(1)$ & $2661(1)$ & 13 \\
& & & & \\
\hline
\end{tabular}


Table 3. Bond lengths [Å] and angles [] for S19177.

\begin{tabular}{|c|c|c|c|}
\hline O1-C4 & $1.4026(15)$ & O1-C5 & $1.4476(16)$ \\
\hline $\mathrm{O} 2-\mathrm{C} 4$ & $1.3975(15)$ & $\mathrm{O} 2-\mathrm{C} 6$ & $1.4366(17)$ \\
\hline O3-C8 & $1.4119(15)$ & $\mathrm{O} 3-\mathrm{H} 4$ & $0.837(14)$ \\
\hline O4-C9 & $1.4549(16)$ & O4-C10 & $1.3462(16)$ \\
\hline $\mathrm{O} 5-\mathrm{C} 3$ & $1.4384(15)$ & O5-C11 & $1.4448(15)$ \\
\hline $\mathrm{O} 6-\mathrm{C} 3$ & $1.3862(15)$ & $\mathrm{O} 6-\mathrm{H} 1$ & $0.831(14)$ \\
\hline O7-C2 & $1.4281(16)$ & $\mathrm{O} 7-\mathrm{H} 3$ & $0.830(14)$ \\
\hline $\mathrm{O}-\mathrm{C} 1$ & $1.4273(16)$ & $\mathrm{O} 8-\mathrm{H} 2$ & $0.837(14)$ \\
\hline O9-C10 & $1.2028(16)$ & $\mathrm{N} 1-\mathrm{C} 7$ & $1.4495(16)$ \\
\hline $\mathrm{N} 1-\mathrm{H} 5$ & $0.863(13)$ & $\mathrm{N} 1-\mathrm{H} 6$ & $0.844(14)$ \\
\hline C1-C2 & $1.5251(18)$ & $\mathrm{C} 1-\mathrm{H} 11$ & $0.978(17)$ \\
\hline $\mathrm{C} 1-\mathrm{H} 12$ & $0.988(17)$ & $\mathrm{C} 2-\mathrm{C} 3$ & $1.5590(17)$ \\
\hline C2-C9 & $1.5480(18)$ & C3-C4 & $1.5468(18)$ \\
\hline C4-C7 & $1.5506(17)$ & $\mathrm{C} 5-\mathrm{H} 51$ & $0.932(18)$ \\
\hline C5-H52 & $0.988(18)$ & C5-H53 & $0.952(18)$ \\
\hline $\mathrm{C} 6-\mathrm{H} 61$ & $0.992(19)$ & $\mathrm{C} 6-\mathrm{H} 62$ & $0.985(19)$ \\
\hline C6-H63 & $0.968(19)$ & C7-C8 & $1.5386(17)$ \\
\hline C7-C11 & $1.5717(18)$ & C8-C9 & $1.5276(19)$ \\
\hline C8-H81 & $0.962(17)$ & $\mathrm{C} 9-\mathrm{H} 91$ & $0.971(17)$ \\
\hline $\mathrm{C} 10-\mathrm{C} 11$ & $1.5210(18)$ & $\mathrm{C} 11-\mathrm{H} 111$ & $0.964(17)$ \\
\hline C4-O1-C5 & $116.79(10)$ & $\mathrm{C} 4-\mathrm{O} 2-\mathrm{C} 6$ & $118.32(10)$ \\
\hline $\mathrm{C} 8-\mathrm{O} 3-\mathrm{H} 4$ & $108.5(13)$ & $\mathrm{C} 9-\mathrm{O} 4-\mathrm{C} 10$ & $116.10(10)$ \\
\hline C3-O5-C11 & $107.53(9)$ & $\mathrm{C} 3-\mathrm{O} 6-\mathrm{H} 1$ & $109.5(13)$ \\
\hline $\mathrm{C} 2-\mathrm{O} 7-\mathrm{H} 3$ & $105.6(13)$ & $\mathrm{C} 1-\mathrm{O} 8-\mathrm{H} 2$ & $103.7(14)$ \\
\hline C7-N1-H5 & $109.7(10)$ & C7-N1-H6 & $113.0(10)$ \\
\hline H5-N1-H6 & $106.0(16)$ & $\mathrm{O} 8-\mathrm{C} 1-\mathrm{C} 2$ & $108.47(11)$ \\
\hline $\mathrm{O} 8-\mathrm{C} 1-\mathrm{H} 11$ & $109.6(10)$ & $\mathrm{C} 2-\mathrm{C} 1-\mathrm{H} 11$ & $109.9(10)$ \\
\hline $\mathrm{O} 8-\mathrm{C} 1-\mathrm{H} 12$ & $110.9(10)$ & $\mathrm{C} 2-\mathrm{C} 1-\mathrm{H} 12$ & $108.8(10)$ \\
\hline $\mathrm{H} 11-\mathrm{C} 1-\mathrm{H} 12$ & $109.2(14)$ & $\mathrm{C} 1-\mathrm{C} 2-\mathrm{O} 7$ & $106.99(10)$ \\
\hline $\mathrm{C} 1-\mathrm{C} 2-\mathrm{C} 3$ & $110.82(10)$ & $\mathrm{O} 7-\mathrm{C} 2-\mathrm{C} 3$ & $111.69(10)$ \\
\hline C1-C2-C9 & $109.04(10)$ & O7-C2-C9 & $107.26(10)$ \\
\hline C3-C2-C9 & $110.88(10)$ & $\mathrm{C} 2-\mathrm{C} 3-\mathrm{O} 5$ & $107.38(10)$ \\
\hline C2-C3-O6 & 112.87(10) & O5-C3-O6 & $109.03(10)$ \\
\hline C2-C3-C4 & $112.73(10)$ & O5-C3-C4 & $101.59(9)$ \\
\hline O6-C3-C4 & $112.44(10)$ & C3-C4-O1 & $107.71(10)$ \\
\hline $\mathrm{C} 3-\mathrm{C} 4-\mathrm{O} 2$ & $113.95(10)$ & $\mathrm{O} 1-\mathrm{C} 4-\mathrm{O} 2$ & $112.89(10)$ \\
\hline C3-C4-C7 & $99.50(9)$ & O1-C4-C7 & $117.79(10)$ \\
\hline $\mathrm{O} 2-\mathrm{C} 4-\mathrm{C} 7$ & $104.48(10)$ & O1-C5-H51 & 106.8(11) \\
\hline O1-C5-H52 & $108.6(11)$ & H51-C5-H52 & $110.9(15)$ \\
\hline $\mathrm{O} 1-\mathrm{C} 5-\mathrm{H} 53$ & $111.2(11)$ & H51-C5-H53 & $108.0(15)$ \\
\hline H52-C5-H53 & $111.2(15)$ & O2-C6-H61 & 103.1(11) \\
\hline O2-C6-H62 & $110.8(11)$ & H61-C6-H62 & $110.0(15)$ \\
\hline O2-C6-H63 & $112.7(11)$ & H61-C6-H63 & $110.8(15)$ \\
\hline H62-C6-H63 & $109.3(15)$ & C4-C7-N1 & $114.68(10)$ \\
\hline C4-C7-C8 & $108.36(10)$ & N1-C7-C8 & $110.50(10)$ \\
\hline C4-C7-C11 & $100.01(10)$ & $\mathrm{N} 1-\mathrm{C} 7-\mathrm{C} 11$ & $114.25(10)$ \\
\hline $\mathrm{C} 8-\mathrm{C} 7-\mathrm{C} 11$ & $108.38(10)$ & C7-C8-O3 & $107.24(10)$ \\
\hline
\end{tabular}




$\begin{array}{lllr}\text { C7-C8-C9 } & 107.42(10) & \text { O3-C8-C9 } & 111.50(10) \\ \text { C7-C8-H81 } & 111.8(10) & \text { O3-C8-H81 } & 110.9(10) \\ \text { C9-C8-H81 } & 108.0(10) & \text { C2-C9-C8 } & 112.18(10) \\ \text { C2-C9-O4 } & 112.74(10) & \text { C8-C9-O4 } & 109.07(10) \\ \text { C2-C9-H91 } & \text { C8-C9-H91 } & 111.3(10) \\ \text { O4-C9-H91 } & 108.6(10) & \text { O4-C10-O9 } & 120.63(12) \\ \text { O4-C10-C11 } & 102.6(10) & \text { O9-C10-C11 } & 124.81(12) \\ \text { C10-C11-C7 } & 114.29(11) & \text { C10-C11-O5 } & 105.02(10) \\ \text { C7-C11-O5 } & 113.69(11) & \text { C10-C11-H111 } & 111.0(10) \\ \text { C7-C11-H111 } & 107.01(10) & \text { O5-C11-H111 } & 108.9(10)\end{array}$

Symmetry transformations used to generate equivalent atoms: 
Table 4. Anisotropic displacement parameters $\left(\AA^{2} \times 10^{3}\right)$ for $\mathbf{S 1 9 1 7 7 .}$ The anisotropic displacement factor exponent takes the form: $-2 \pi^{2}\left[h^{2} a^{* 2} U^{11}+\ldots+2 h k a^{*} b^{*} U^{12}\right]$

\begin{tabular}{lcccccc}
\hline & $U^{11}$ & $U^{22}$ & $U^{33}$ & $U^{23}$ & $U^{13}$ & $U^{12}$ \\
\hline O1 & $13(1)$ & $13(1)$ & $14(1)$ & $-3(1)$ & $1(1)$ & $-2(1)$ \\
O2 & $19(1)$ & $13(1)$ & $13(1)$ & $2(1)$ & $5(1)$ & $-1(1)$ \\
O3 & $18(1)$ & $15(1)$ & $14(1)$ & $2(1)$ & $0(1)$ & $6(1)$ \\
O4 & $16(1)$ & $12(1)$ & $18(1)$ & $1(1)$ & $3(1)$ & $-1(1)$ \\
O5 & $14(1)$ & $12(1)$ & $13(1)$ & $-2(1)$ & $-1(1)$ & $1(1)$ \\
O6 & $12(1)$ & $14(1)$ & $15(1)$ & $3(1)$ & $1(1)$ & $3(1)$ \\
O7 & $16(1)$ & $16(1)$ & $14(1)$ & $-2(1)$ & $4(1)$ & $-1(1)$ \\
O8 & $10(1)$ & $17(1)$ & $29(1)$ & $1(1)$ & $2(1)$ & $1(1)$ \\
O9 & $22(1)$ & $13(1)$ & $22(1)$ & $-2(1)$ & $0(1)$ & $-1(1)$ \\
N1 & $12(1)$ & $17(1)$ & $15(1)$ & $0(1)$ & $3(1)$ & $2(1)$ \\
C1 & $12(1)$ & $15(1)$ & $19(1)$ & $2(1)$ & $3(1)$ & $1(1)$ \\
C2 & $12(1)$ & $13(1)$ & $12(1)$ & $0(1)$ & $2(1)$ & $1(1)$ \\
C3 & $12(1)$ & $10(1)$ & $11(1)$ & $0(1)$ & $0(1)$ & $1(1)$ \\
C4 & $13(1)$ & $12(1)$ & $10(1)$ & $-1(1)$ & $2(1)$ & $-1(1)$ \\
C5 & $13(1)$ & $17(1)$ & $21(1)$ & $-4(1)$ & $-1(1)$ & $-1(1)$ \\
C6 & $23(1)$ & $15(1)$ & $22(1)$ & $6(1)$ & $3(1)$ & $-1(1)$ \\
C7 & $11(1)$ & $11(1)$ & $12(1)$ & $-1(1)$ & $1(1)$ & $1(1)$ \\
C8 & $14(1)$ & $12(1)$ & $12(1)$ & $0(1)$ & $0(1)$ & $2(1)$ \\
C9 & $16(1)$ & $12(1)$ & $13(1)$ & $1(1)$ & $2(1)$ & $1(1)$ \\
C10 & $12(1)$ & $14(1)$ & $16(1)$ & $1(1)$ & $0(1)$ & $1(1)$ \\
C11 & $13(1)$ & $12(1)$ & $13(1)$ & $-1(1)$ & $1(1)$ & $1(1)$ \\
& & & & & & \\
\hline
\end{tabular}


Table 5. Hydrogen coordinates $\left(\times 10^{4}\right)$ and isotropic displacement parameters $\left(\AA^{2} \times 10^{3}\right)$ for S19177.

\begin{tabular}{lrrrr}
\hline & $x$ & $y$ & $\mathrm{U}(\mathrm{eq})$ \\
$\mathrm{H} 11$ & & & \\
H12 & & & \\
H51 & $1660(20)$ & $7081(12)$ & $5234(16)$ & 18 \\
H52 & $1770(20)$ & $6887(12)$ & $3750(17)$ & 18 \\
H53 & $7850(20)$ & $9892(13)$ & $5197(17)$ & 21 \\
H61 & $8320(20)$ & $9433(13)$ & $3916(18)$ & 21 \\
H62 & $8410(20)$ & $8834(13)$ & $5224(17)$ & 21 \\
H63 & $6810(20)$ & $9820(13)$ & $1280(18)$ & 24 \\
H81 & $6090(20)$ & $10120(14)$ & $2579(18)$ & 24 \\
H91 & $4950(20)$ & $9718(13)$ & $1395(18)$ & 24 \\
H111 & $6760(20)$ & $7492(12)$ & $5584(16)$ & 16 \\
H1 & $4480(20)$ & $6630(12)$ & $5908(16)$ & 16 \\
H2 & $5830(20)$ & $6610(12)$ & $1868(16)$ & 14 \\
H3 & $2310(18)$ & $8834(13)$ & $3131(17)$ & $24(2)$ \\
H4 & $101(19)$ & $7987(14)$ & $3888(18)$ & $31(2)$ \\
H5 & $4556(19)$ & $8753(13)$ & $5442(18)$ & $26(2)$ \\
H6 & $7560(20)$ & $6074(14)$ & $5983(14)$ & $27(2)$ \\
& $8612(18)$ & $6702(11)$ & $3656(16)$ & $19(2)$ \\
\hline & $8315(17)$ & $7219(13)$ & $2552(13)$ & $20(2)$ \\
\hline
\end{tabular}


Table 6. Torsion angles $\left[{ }^{\circ}\right]$ for S19177.

\begin{tabular}{|c|c|c|c|}
\hline C5-O1-C4-O2 & $-58.33(14)$ & C5-O1-C4-C3 & $174.97(10)$ \\
\hline C5-O1-C4-C7 & $63.63(15)$ & $\mathrm{C} 6-\mathrm{O} 2-\mathrm{C} 4-\mathrm{O} 1$ & $-46.99(15)$ \\
\hline $\mathrm{C} 6-\mathrm{O} 2-\mathrm{C} 4-\mathrm{C} 3$ & $76.31(14)$ & $\mathrm{C} 6-\mathrm{O} 2-\mathrm{C} 4-\mathrm{C} 7$ & $-176.17(11)$ \\
\hline C10-O4-C9-C2 & $84.84(13)$ & C10-O4-C9-C8 & $-40.46(14)$ \\
\hline C9-O4-C10-O9 & $164.47(12)$ & C9-O4-C10-C11 & $-21.13(15)$ \\
\hline C11-O5-C3-O6 & $157.90(10)$ & C11-O5-C3-C2 & $-79.50(12)$ \\
\hline C11-O5-C3-C4 & $39.03(12)$ & C3-O5-C11-C7 & $-13.00(12)$ \\
\hline C3-O5-C11-C10 & $108.14(11)$ & $\mathrm{O} 8-\mathrm{C} 1-\mathrm{C} 2-\mathrm{O} 7$ & $58.94(13)$ \\
\hline $\mathrm{O} 8-\mathrm{C} 1-\mathrm{C} 2-\mathrm{C} 3$ & $-63.05(13)$ & O8-C1-C2-C9 & $174.63(10)$ \\
\hline O7-C2-C3-O5 & $176.27(10)$ & O7-C2-C3-O6 & $-63.53(14)$ \\
\hline O7-C2-C3-C4 & $65.21(13)$ & C1-C2-C3-O5 & $-64.54(13)$ \\
\hline $\mathrm{C} 1-\mathrm{C} 2-\mathrm{C} 3-\mathrm{O} 6$ & $55.66(14)$ & $\mathrm{C} 1-\mathrm{C} 2-\mathrm{C} 3-\mathrm{C} 4$ & $-175.61(10)$ \\
\hline C9-C2-C3-O5 & $56.70(13)$ & C9-C2-C3-O6 & $176.90(10)$ \\
\hline C9-C2-C3-C4 & $-54.36(13)$ & $\mathrm{O} 7-\mathrm{C} 2-\mathrm{C} 9-\mathrm{O} 4$ & 160.41(10) \\
\hline O7-C2-C9-C8 & $-75.99(13)$ & C1-C2-C9-O4 & $44.89(14)$ \\
\hline C1-C2-C9-C8 & $168.49(10)$ & C3-C2-C9-O4 & $-77.39(13)$ \\
\hline C3-C2-C9-C8 & $46.21(14)$ & $\mathrm{O} 5-\mathrm{C} 3-\mathrm{C} 4-\mathrm{O} 1$ & $-172.60(9)$ \\
\hline O5-C3-C4-O2 & $61.33(12)$ & O5-C3-C4-C7 & $-49.26(11)$ \\
\hline O6-C3-C4-O1 & $71.00(13)$ & $\mathrm{O} 6-\mathrm{C} 3-\mathrm{C} 4-\mathrm{O} 2$ & $-55.08(14)$ \\
\hline $\mathrm{O} 6-\mathrm{C} 3-\mathrm{C} 4-\mathrm{C} 7$ & $-165.67(10)$ & $\mathrm{C} 2-\mathrm{C} 3-\mathrm{C} 4-\mathrm{O} 1$ & $-57.97(13)$ \\
\hline C2-C3-C4-O2 & $175.95(10)$ & C2-C3-C4-C7 & $65.36(12)$ \\
\hline O1-C4-C7-N1 & $-81.77(14)$ & O1-C4-C7-C8 & $42.20(15)$ \\
\hline O1-C4-C7-C11 & $155.53(11)$ & O2-C4-C7-N1 & $44.41(14)$ \\
\hline $\mathrm{O} 2-\mathrm{C} 4-\mathrm{C} 7-\mathrm{C} 8$ & $168.38(10)$ & $\mathrm{O} 2-\mathrm{C} 4-\mathrm{C} 7-\mathrm{C} 11$ & $-78.29(11)$ \\
\hline C3-C4-C7-N1 & $162.34(10)$ & C3-C4-C7-C8 & $-73.69(11)$ \\
\hline C3-C4-C7-C11 & $39.63(11)$ & $\mathrm{N} 1-\mathrm{C} 7-\mathrm{C} 8-\mathrm{O} 3$ & $-42.69(13)$ \\
\hline N1-C7-C8-C9 & $-162.66(10)$ & $\mathrm{C} 4-\mathrm{C} 7-\mathrm{C} 8-\mathrm{O} 3$ & $-169.13(10)$ \\
\hline $\mathrm{C} 4-\mathrm{C} 7-\mathrm{C} 8-\mathrm{C} 9$ & $70.90(12)$ & $\mathrm{C} 11-\mathrm{C} 7-\mathrm{C} 8-\mathrm{O} 3$ & $83.21(12)$ \\
\hline C11-C7-C8-C9 & $-36.76(13)$ & N1-C7-C11-O5 & $-141.12(11)$ \\
\hline N1-C7-C11-C10 & $103.40(13)$ & C4-C7-C11-O5 & $-18.11(12)$ \\
\hline C4-C7-C11-C10 & $-133.59(11)$ & C8-C7-C11-O5 & $95.20(11)$ \\
\hline C8-C7-C11-C10 & $-20.27(14)$ & O3-C8-C9-O4 & $-45.64(13)$ \\
\hline O3-C8-C9-C2 & $-171.27(10)$ & C7-C8-C9-O4 & $71.58(12)$ \\
\hline C7-C8-C9-C2 & $-54.05(13)$ & O4-C10-C11-O5 & $-62.35(13)$ \\
\hline O4-C10-C11-C7 & $54.29(15)$ & O9-C10-C11-O5 & $111.78(14)$ \\
\hline O9-C10-C11-C7 & $-131.58(14)$ & & \\
\hline
\end{tabular}

Symmetry transformations used to generate equivalent atoms: 